\title{
TD-DFT: An Exploration of the Energies and Structures of Crystal Violet and a Variety of Cr(III) Complex Ions
}

Richard Kleingeld

Supervisor: Dr Matthias Lein

Research project in consideration towards a Master's degree in Chemistry 2013 


\section{Contents}

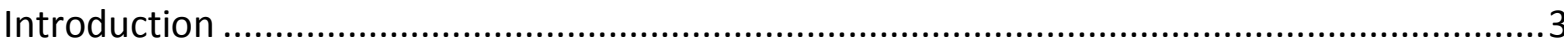

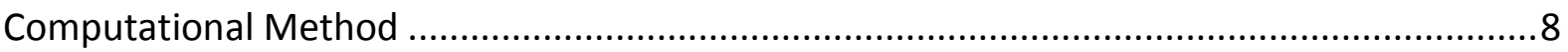

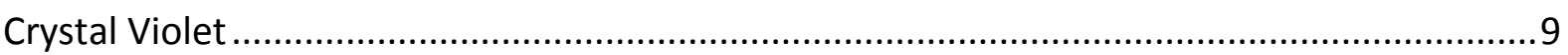

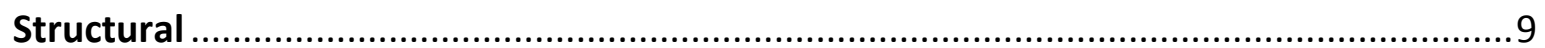

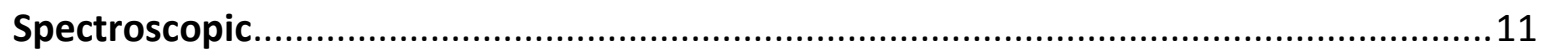

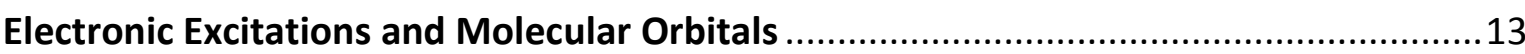

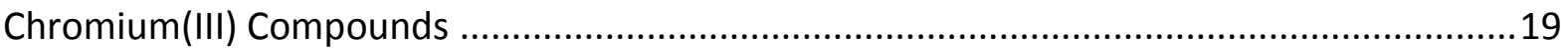

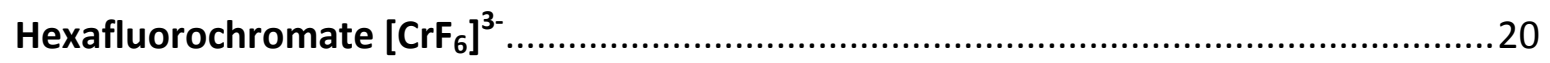

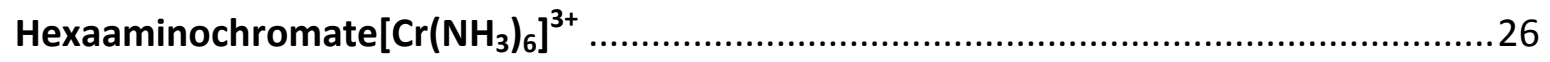

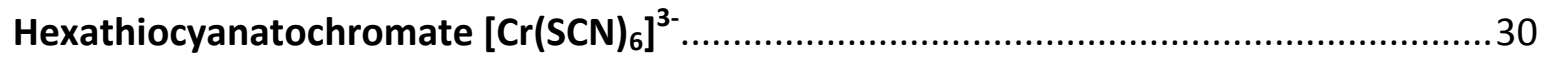

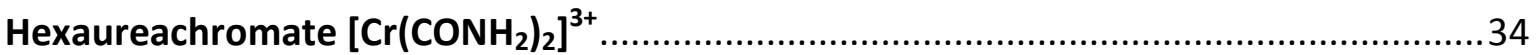

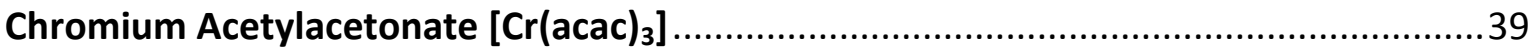

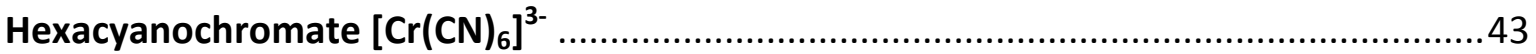

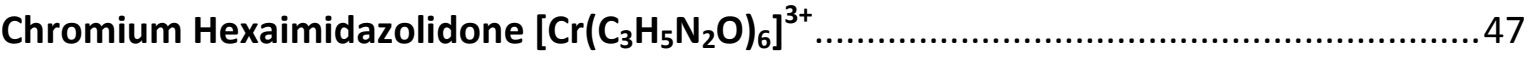

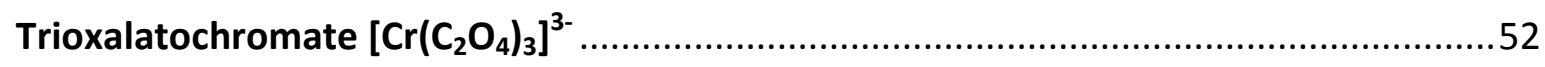

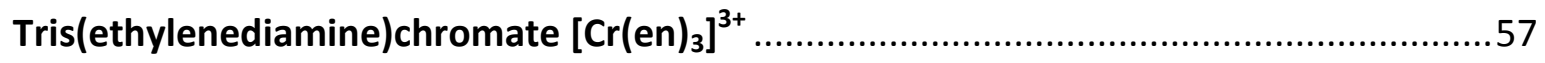

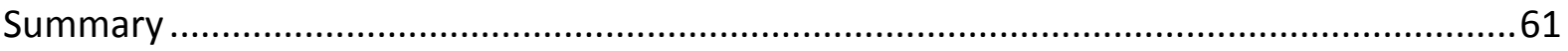

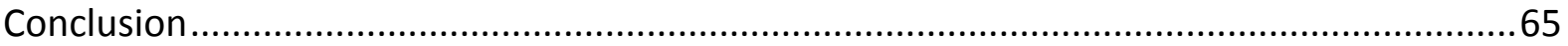

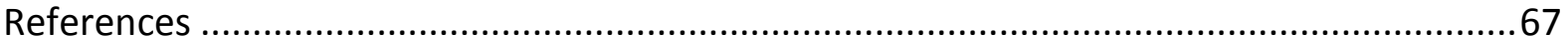




\section{Introduction}

Spectroscopy is the science of utilising light in order to divine information about a molecule or system of molecules. Specifically, the absorption, emission, and scattering of different wavelengths of light can provide data about bond strength, bond order, vibrational frequency, and excitation energy [1, 2]. As the wavelength and therefore energy of the incident photons can be set by the instrument, the exact energies of absorbance or emission of the molecule can be measured. This data can be gathered experimentally using specialised equipment however some molecules resist synthesis, and so a wealth of data about many theoretically possible species eludes us. We may also want to isolate the molecule in "empty space" whereas "gas phase" measurements are not always possible. This is one place where computational chemistry comes to the fore. Using an appropriate computational method such as density functional theory (DFT), data can be theoretically derived and calculated for many interesting areas of chemistry.

DFT is a computational method based on the findings of Hohenberg and Kohn in 1964 that the ground state electronic energy of a system can be determined completely by the electron density [3-6]. This means that it has a considerably higher efficiency as a computational method compared to the wave function approach, where the number of variables increases exponentially as your system increases in size, as the electron density has the same number of variables regardless of the size of the system [7]. The use of an appropriate functional to map the electron density and the energy is one of the vital choices in utilising this method, but if chosen well can provide good results with a much lower computational cost than other methods, while still accounting for electron correlation effects [8]. It has become a very popular method due to its versatility and generally good accuracy with relatively low computational expense when compared to $a b$ initio methods [9].

The DFT method was extended into the time dependent domain by Runge and Gross in 1984 [9], and expanded by the development of a linear-response formulation by Casida whereby a TD-DFT expression for the dynamic polarisability is derived, and the sum-overstates formula shown below (Equation 1) is used to obtain the excitation energies and oscillator strengths from the dynamic polarisability [10]. 


$$
\bar{\alpha}(\omega)=\frac{1}{3} \operatorname{tr} \alpha(\omega)=\sum_{I} \frac{f_{I}}{\omega_{I}^{2}-\omega^{2}}
$$

Equation 1: Sum over states formula used to obtain energies and oscillator strengths from the dynamic polarisability $(\omega)$

As this allows for the application of TD-DFT for dynamic molecular applications, this method has been used extensively in order to examine the energies, as well as electronic, geometric and optical properties of molecular excited states [4, 11-17]. It is important to manage computational cost carefully when performing excited state calculations as they often require more computational resources and are more complicated than ground state calculations. Some applications of TD-DFT are the calculation of vertical excitation energies [18], simulation of optical spectra [19] and photochemical reactions [20], as well as the computation of excited state structures and emission wavelengths [21].

In chemistry the wave function for a given compound can be constructed using the linear combination of atomic orbitals approach. This is where the atomic orbitals of the atoms composing the molecule are used to build its molecular orbitals. In the simplest approximation, MO theory uses the occupied MOs to construct a single Slater Determinant which is used as the wavefunction to compute the energy. This construction from a single configuration is usually very accurate; however it can be improved by including more than the ground state configuration. This is called Configuration Interaction $(\mathrm{Cl})$ method. When investigating excited states of molecules, the 'excited determinants' are constructed by exchanging MOs which are occupied in the ground state (GS) with those which are not, effectively promoting an electron between orbitals. In many cases a single determinant is of particular importance to the excited state and so the single electronic transition model is effective. For some systems, however, more than one excited configuration is important in describing the excitation, and so the simple one electron picture breaks down and contributions from multiple electronic configurations must be discussed [7]. Vertical excitation energies ( $E_{\text {vert }}$ ) show the energy difference between the ground and excited states of a molecule for a given molecular geometry (Figure 1a). If the excitation is made from the equilibrium structure of the ground state, then a prediction of the UV-Vis spectrum for that molecule can be made. Comparison of the theoretical spectra to experimental results gives an indication of the accuracy of the computational model employed. This process also identifies the electronic transitions between molecular orbitals for each calculated excited 
state, which means that the geometry of the excited state can be predicted and explained, along with chemical and physical effects caused by the electronic excitations. These effects include fluorescence or phosphorescence through emission of absorbed energy, changes in structure which can affect physical characteristics such as melting or boiling point, and intersystem crossing can lead to applications in solar cells $[22,23]$. The calculation of molecular orbitals is an effective tool for the prediction of the structure of excited states. Simply by modelling the molecular orbitals, a visual analysis of the molecular orbitals involved in an electronic transition can be performed. As these transitions are responsible for the promotion of electrons into an excited state configuration, by comparing the MO from which the electron is excited to the $\mathrm{MO}$ in which it resides after excitation, predictions can be made about the excited state. This can be done simply by looking at where there are bonding, non-bonding and antibonding interactions between atoms in the two molecular orbitals and noting changes in mode of interaction and intensity, as well as the movement of charge density.

The chemical and physical effects caused by molecular excitation can be utilised in order to design materials for specific applications [24-27]. The optimised geometric structure of the excited state can also be calculated using TD-DFT, which is beneficial when considering photochemical reactions, as the excited state may be more favourably disposed towards a particular reaction than in the ground state.

The calculation of the various excited states of a molecule is useful in the field of spectroscopy, as it allows the prediction of absorption (Figure 1a), emission/fluorescence (Figure 1c) and phosphorescence (Figure 1e) spectra for that molecule, just as similar calculations of the ground state can provide simulated vibrational and scattering spectra. This can be especially useful when considering molecules or systems which have not yet been synthesized and so have no experimental data associated with them. In this case, a theoretical model of the molecule lets us make predictions about the chemistry of the molecule, such as the associated spectra and minimum energy structural information, and can assist in creating a synthetic scheme. For molecules or systems which have been experimentally synthesized, information which is not directly measurable can be obtained, such as transition dipole moments for electronic transitions. This aids in identifying and characterising specific transitions in spectroscopic experiments. 


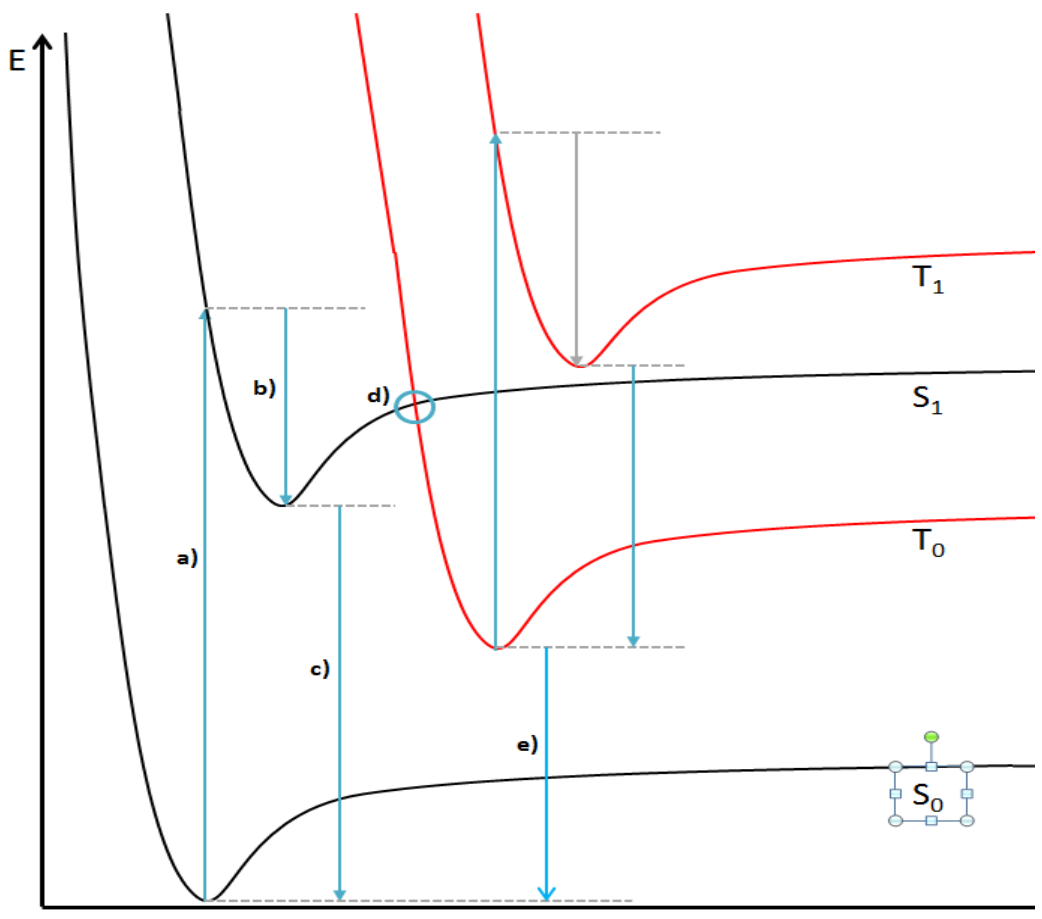

Figure 1: Diagram of some electronic transitions showing: a) Absorption b) Vibrational relaxation c) Fluorescence d) Intersystem crossing e) Phosphorescence

In this work, two types of compound have been investigated using TD-DFT. The first is an organic dye, in this case crystal violet (CV) (Figure 2).

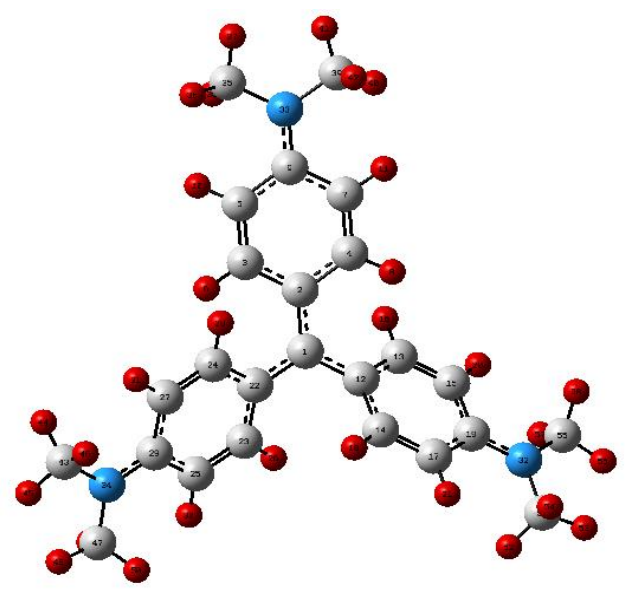

Figure 2: Crystal Violet

This is a tri-p-dimethylaminophenyl carbonium ion with a "propeller" shape of $D_{3}$ symmetry, with the aromatic rings angled at about $33^{\circ}$ off the plane of the carbonium centre $[28,29]$. However, there are differing opinions as to its exact conformation and chemical activity in the liquid phase, or when paired with certain counter ions. Meng et al. have asserted that due to steric hindrance, crystal violet maintains its normal $D_{3}$ symmetry 
as both a crystalline solid and in solution [29]. Liang et al. utilised surface enhanced raman spectroscopy (SERS) to analyse the effect on CV of halide and halate ions [30]. They found that the ions coordinate to the central positively charged carbon causing electron density to increase in the phenyl ring and the N-Ph bonds which has a major effect on the vibrational modes on the molecule. They also assert that CV adsorbs to a silver substrate through the nitrogen lone pairs [30]. This kind of adsorption would likely sterically restrict the angle of the phenyl rings. As a dye, crystal violet is commonly used in biological studies as a Gram stain, and has been widely used as a rigorous test for SERS due to its reasonably high symmetry and excellent adsorption onto substrate surfaces [28]. Both the singlet ground state and lowest lying triplet state of this molecule were investigated.

The other type of compound analysed was a series of $\mathrm{Cr}(\mathrm{III})$ complexes with a variety of ligands. The ligands were chosen to represent strong and weak field ligands, as well as mono- and bi-dentate ligands. $\mathrm{Cr}(\mathrm{III})$ forms $\mathrm{d}^{3}$ complexes and has a quartet ${ }^{4} \mathrm{~F}$ ground state as well as a higher energy doublet state, the lowest lying of which is ${ }^{2} \mathrm{G}$ (Figure 3) [31]. A number of experimentally synthesised $\mathrm{Cr}$ (III) compounds, as well as some which are theoretically possible but have not been successfully synthesised, were studied. There is little computational work done directly on many of these simpler chromium complexes available in the literature. Many of the experimental works are decades old, dating back to the early synthesis and characterisation of the compounds in the mid-20 th century [32-34]. Due to its interesting and unusual electronic structure, chromium can present a computational challenge, which can be mitigated through careful selection of method and basis set. In this work the quartet and doublet ground states of each $\mathrm{Cr}$ (III) compound have been investigated. The quartet is the most stable ground state for $\mathrm{Cr}(\mathrm{III})$ compounds but for some ligand systems it is possible for the difference in energy between the quartet and doublet states to be fairly small. For this reason it is important to model the compounds in both states to ensure that the spectroscopic transitions observed are indeed from the quartet state. Also, due to the possibility of intersystem crossing as depicted in Figure $1 \mathrm{~d}$, the doublet state may lie in an energetic position such that intersystem crossing is possible leading to phosphorescence. 


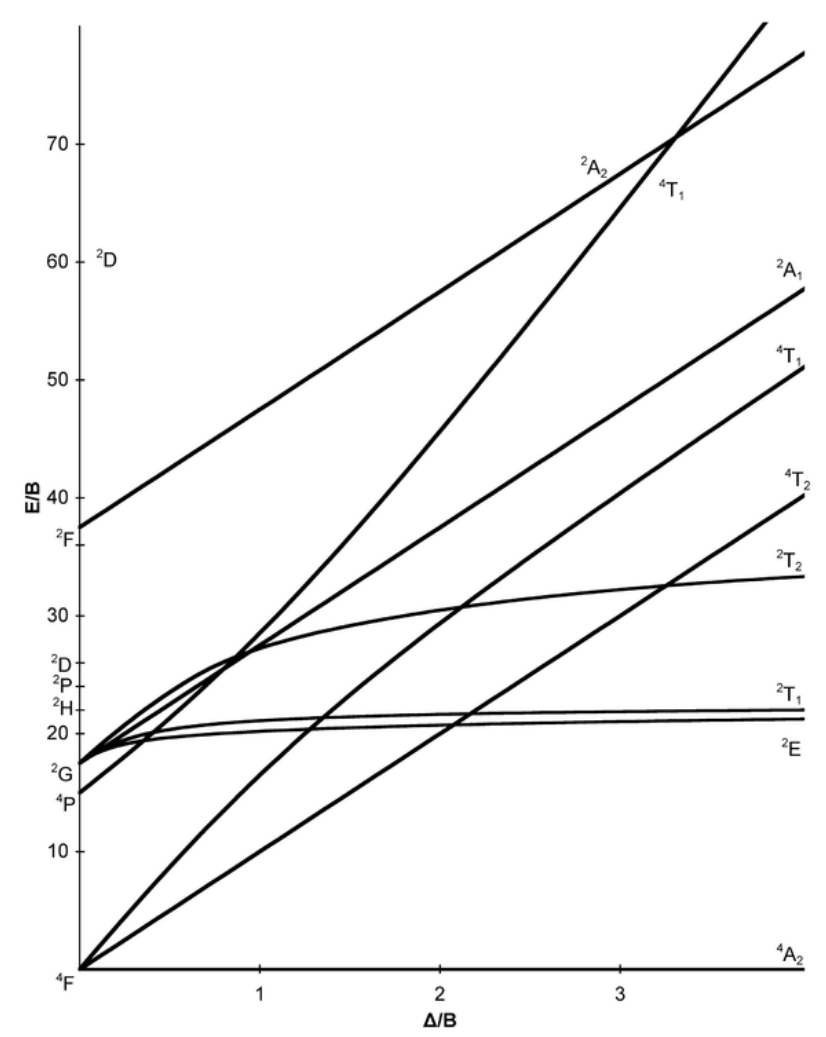

Figure 3: Tanabe-Sugano diagram for $d_{3}$ octahedral complex [35]

This work examines the structures and energies of the ground state (GS) and first excited state (ES) of these two types of compound. We also predict the UV-Vis spectra of the compounds and, where available, compare them to experimental data.

\section{Computational Method}

All calculations were performed using the Gaussian09 computational package [36] utilising a large basis set of triple-zeta quality. The ground states were optimised using the X3LYP/6-311G(d) level of theory [37], as this method was shown to have a low error when calculating polyaromatic hydrocarbons and similar molecules such as CV [38]. The vertical excitation energies were then calculated using TD-DFT for the first 12 excited states of crystal violet and the first 60 excited states of each chromium compound. The optimised geometry of the first excited state was then calculated and the energy differences determined. Harmonic frequencies were calculated for all reported stationary points and they were all determined to be true minima as no imaginary frequencies were found. All visualisations and images of molecules and molecular orbitals were generated using Gaussview v5.0.9 [39]. The atomic charges were calculated using the Mulliken method. 
All molecules were initially modelled in the gas phase, however some were remodelled in aqueous solution for better comparison to experimental data. This was done using the integral equation formalism polarizable continuum model (IEFPCM) for solvation whereby the solvent cavity is modelled via a set of overlapping spheres [40].

The geometries of all molecules were calculated with no symmetry restrictions, however where the resulting structure was found to be close to a higher symmetry then the molecule was constrained to this symmetry and the energies recalculated to see if the higher symmetry was energetically reasonable or within the computational error, and hence whether it represented a distinct separate structure.

In order to assess the general suitability of our chosen method a small benchmarking experiment was conducted, where single point energies of the quartet state of the hexafluorochromate molecule were calculated using CISD methods. The X3LYP method used in the main experiment was compared to these other methods for a number of different sized basis sets ranging from cc-VTZp to cc-V5Zp in size. This data is discussed under the hexafluorochromate section.

\section{Crystal Violet}

\section{Structural}

The singlet ground and first excited states of the crystal violet molecule were modelled and optimised, using the results gathered in previous work to suggest a reasonable starting geometry[28, 29, 41]. Their resulting 'propeller' type conformation of the molecule was confirmed by our calculations, with the positive charge of 0.25 positioned primarily at the central carbon (see Table 1), and with the molecule's arms all at an angle of $33^{\circ}$ off the molecular plane (Figure 4a).

The molecule was calculated to possess no symmetry after geometry optimisation, but visibly resembled the $D_{3}$ propeller shape. When the molecule was constrained to $D_{3}$ symmetry and vibrational frequencies calculated, the RMS forces and vibrational modes showed that the $D_{3}$ symmetrised structure was at the same energy within the numerical accuracy of the method. The three arms of the molecule were designated $A, B$ and $C$ with the $\mathrm{C} 1-\mathrm{C} 2$ bond being Arm $\mathrm{A}$, the $\mathrm{C} 1-\mathrm{C} 12$ bond Arm B, and the $\mathrm{C} 1-\mathrm{C} 22$ bond Arm C. It can be 
seen in Figure $4 a$ that the singlet state has all three arms equally conjugated through the carbonium centre.

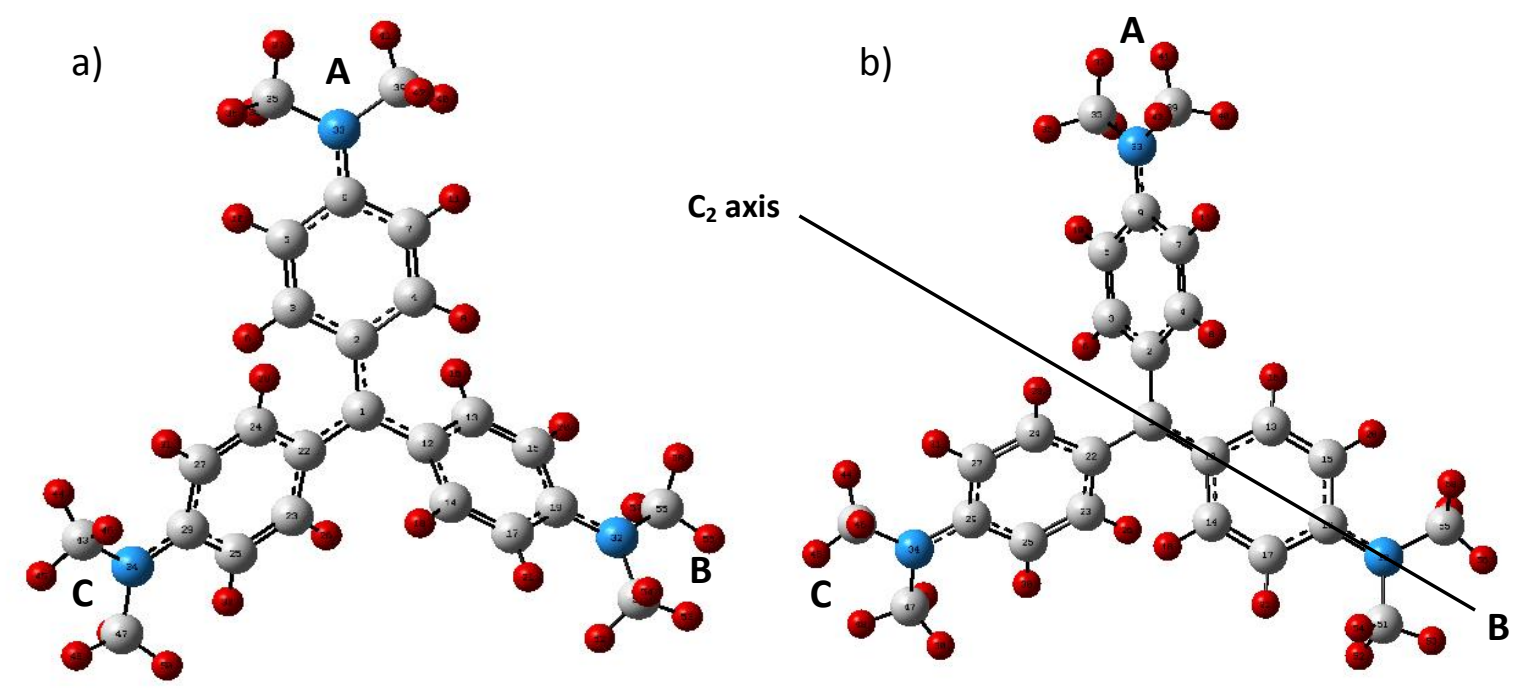

Figure 4: Singlet (a) and triplet (b) ground state optimised structures

The lowest lying triplet state has a similar 'propeller' type structure, but the angle between the arms and the molecular plane is not regular as it was in the singlet state. The angle of Arm $B$ is $19^{\circ}$, much smaller than the angles of the other two arms. Also, arms $A$ and $C$ show slightly different angles above and below the plane, which suggests some distortion of the phenyl ring. Because of these irregularities, the lowest lying triplet state does not have the $D_{3}$ symmetry of the singlet GS and instead shows $C_{2}$ symmetry, with the $C_{2}$ axis running down the centre of Arm B (Figure 4b). The bonding between C1 and the arms is also no longer regular. Instead it shows a single bond to arms $A$ and $C$, and a stronger conjugated bond to Arm B. This is confirmed by the bond lengths shown in Table 1, where the C1-arm A and $C$ length is increased compared to the singlet state, and the C1-Arm B length is decreased slightly. It also exhibits a less positive central carbon with a charge of 0.12 , with Arm $B$ being less negative overall than the other two arms and the negative charge of -0.1 on $\mathrm{C} 12$ which was observed in the singlet state in this case being a positive charge of 0.01 in the triplet state. There is also a general decrease in the negative charge on some of the phenyl carbons, suggesting a higher electron density on the central carbon. Structural information is provided in Table 1, comparing some of the relevant data for both singlet and triplet CV. 
Table 1: Comparison of some relevant structural data for the singlet and triplet ground states of CV. The two values for triplet arm angle denote variation in the clockwise and anticlockwise dihedral angle due to some distortion of the phenyl ring.

\begin{tabular}{|l|ll|}
\hline & Singlet GS & Triplet GS \\
\hline Arm Angle & & \\
Arm A & $33^{\circ}$ & $44^{\circ} / 41^{\circ}$ \\
Arm B & $33^{\circ}$ & $19^{\circ}$ \\
Arm C & $33^{\circ}$ & $44^{\circ} / 41^{\circ}$ \\
\hline C1-Ph length & & \\
Arm A & $1.44 \AA$ & $1.47 \AA$ \\
Arm B & $1.44 \AA$ & $1.43 \AA$ \\
Arm C & $1.44 \AA$ & $1.47 \AA$ \\
\hline Ph-N bond Length & & \\
Arm A & $1.36 \AA$ & $1.37 \AA$ \\
Arm B & $1.36 \AA$ & $1.37 \AA$ \\
Arm C & $1.36 \AA$ & $1.37 \AA$ \\
\hline Atomic Charge (Mulliken) & & \\
C1 & 0.25 & 0.12 \\
C2 & -0.10 & -0.08 \\
C12 & -0.10 & 0.01 \\
C22 & -0.10 & -0.08 \\
N33 (Arm 1) & -0.40 & -0.39 \\
N32 (Arm 2) & -0.40 & -0.37 \\
N34 (Arm 3) & -0.40 & -0.39 \\
\hline
\end{tabular}

\section{Spectroscopic}

The first twelve excited states of both the singlet and triplet were calculated in the gas phase to provide a prediction of the UV/Vis spectrum of crystal violet. This is provided in the form of line spectra with enveloping Gaussian functions of $0.333 \mathrm{eV}$ half width at half height. Both multiplicity states have experimental data for comparison, and the accuracy of the singlet spectrum provides a measure of the reliability in the results obtained for the triplet state. The predicted singlet UV/Vis spectrum has two peaks, one at $477 \mathrm{~nm}$ with $\varepsilon=57500$ and one at $265 \mathrm{~nm}$ with $\varepsilon=17500$. The lower energy peak in the singlet spectrum is resultant of the first excited state transition (Spectrum 1). The triplet first excited state doesn't appear on the spectrum as it occurs at $5984 \mathrm{~nm}$ with an $\varepsilon$ of just 900 which indicates a very small energy gap between the triplet GS and first ES. The visible peaks are a very broad peak at $1180 \mathrm{~nm}$ with $\varepsilon=9100$ and a sharper peak at $452 \mathrm{~nm}$ with $\varepsilon=15500$ (Spectrum 1). In a spectroscopic context, epsilon $(\varepsilon)$ is the molar extinction coefficient which is an intrinsic property of a chemical species and measures how strongly it absorbs light of a particular wavelength. Thus in the case of singlet CV, for example, the species absorbs at $477 \mathrm{~nm} 3.3$ times more strongly than at $265 \mathrm{~nm}$ [42]. The triplet CV experimental data describes an absorption at $410 \mathrm{~nm}$ which can be seen in our predicted spectrum arising at 
$452 \mathrm{~nm}$. This difference may arise from the fact that the triplet CV was modelled in the gas phase while the experimental data had CV solvated in a mixture of acetonitrile, $t$-butanol and benzene [43].

The experimental data for crystal violet in aqueous solution shows one peak at $590 \mathrm{~nm}$ and a smaller peak at $545 \mathrm{~nm}$ [41]. Remodelling the excitation in aqueous solution using the IEFPCM model shifted the theoretical peaks from $477 \mathrm{~nm}$ and $260 \mathrm{~nm}$, to $498 \mathrm{~nm}$ and $265 \mathrm{~nm}$. This corresponds to a difference between the predicted data and the experimental data of $0.39 \mathrm{eV}$ which is somewhat larger than expected for the X3LYP method from a recent benchmark study [44]. That study predicted that the mean average error for X3LYP is 0.22 $\mathrm{eV}$ which would correspond to an acceptable theoretical result in the range of $534 \mathrm{~nm}$ to $659 \mathrm{~nm}$.

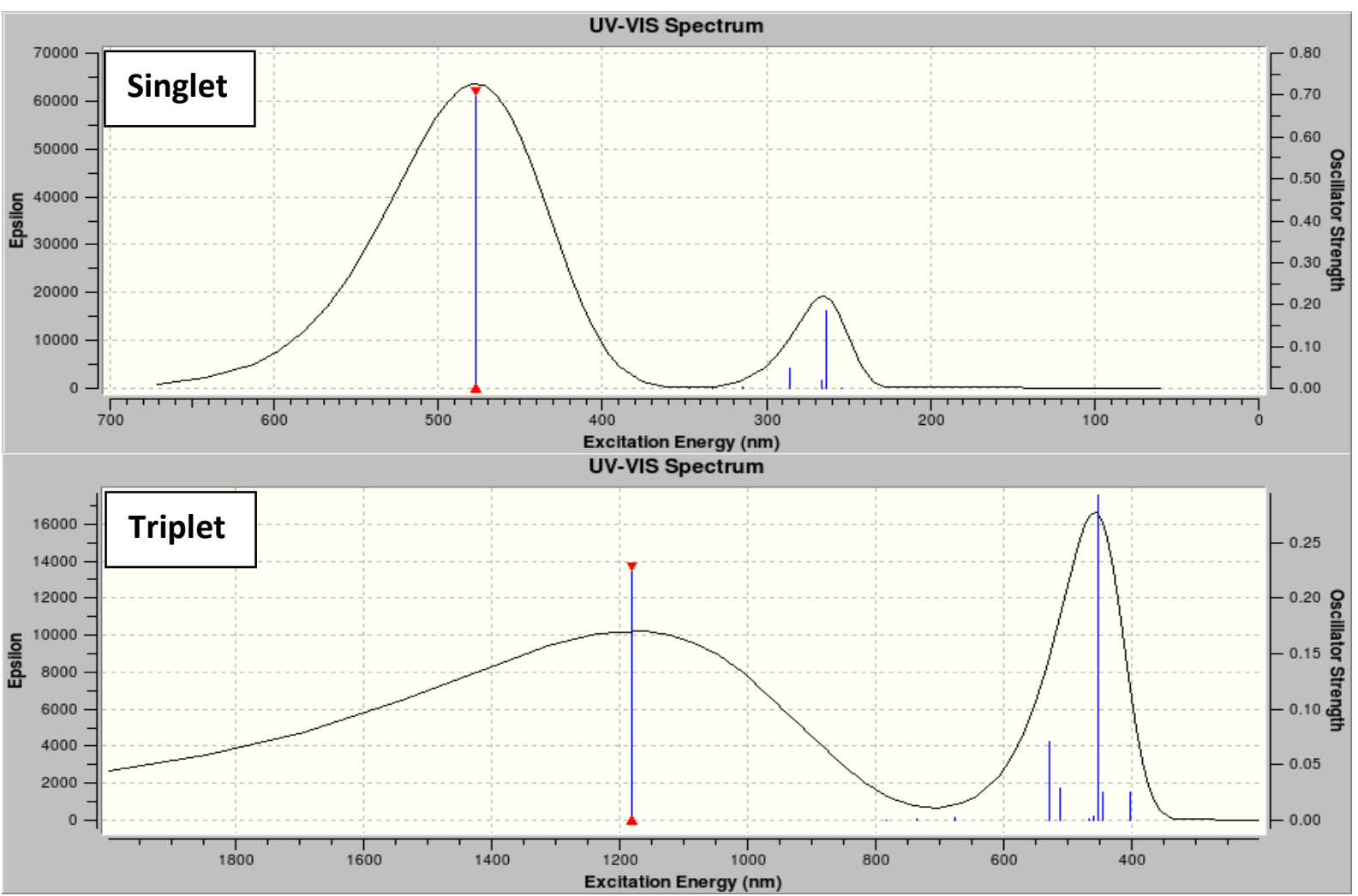

Spectrum 1: Predicted UV-Vis spectra for crystal violet (NB: Due to the large disparity in extinction coefficient between multiplicities, the axes are not to scale) 


\section{Electronic Excitations and Molecular Orbitals}

The first excited state of the singlet and the next higher triplet excited state were examined more closely. The electronic configurations from which the excited states are composed were determined, and the relevant MOs were visually analysed. The singlet first excited state is composed of 2 electronic configurations from the two degenerate MOs which make up the HOMO to the LUMO (Figure 5). This transition corresponds to the main peak observed in the predicted UV/Vis spectrum at $477 \mathrm{~nm}$.

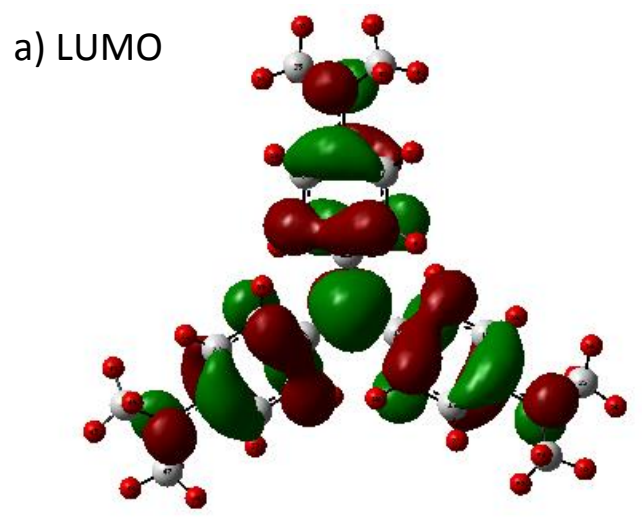

b) HOMO
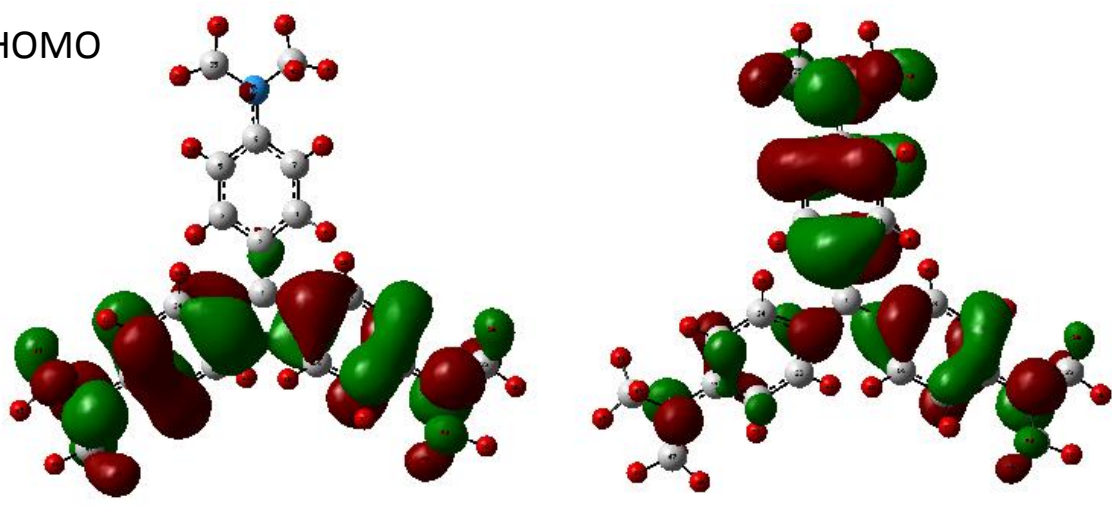

Figure 5: Relevant ground state MOs for CV singlet: a) LUMO b) HOMO

The electronic configuration resulting from the HOMO $\rightarrow$ LUMO transitions affects the geometry of the ES. The MO on Arm A changes phase and there is a notable increase in electron density on the central carbon in the LUMO. There will also be an increase in electron density on Arm A. The most important change is that in the HOMO, there is a bonding interaction between the lobes on C12 and C22. This occurs because although the like phases are on opposite faces of the molecule on each arm, the $33^{\circ}$ angle allows the like phase lobes to interact. This bonding interaction is not present in the LUMO, and it is possible that the loss of this bonding between arms $B$ and $C$ is what allows Arm $B$ to rotate 
to almost $90^{\circ}$ off-plane. This may occur to minimise steric effects between the phenyl rings which were previously compensated for by the bonding interaction.

The optimised geometry of the singlet excited state was also calculated, and it was found that there was a rotation of the arms relative to the molecular plane. This destroyed the $D_{3}$ symmetry of the molecule leaving it with no symmetry, though it is close to being $C_{2}$ along the same axis as the triplet GS (Figure 6). Also, in the excited state geometry, the C1-C12 bond is no longer conjugated to the rest of the molecule and is instead a longer single bond. This bonding causes a change in the charge on the central carbon. Where previously the charge on C1 was 0.25 , it is now - 0.04 , and the charges on C2 and C22 have increased from 0.099 to 0.059 . This means that while in the GS the positive charge was localised on $\mathrm{C} 1$, in the ES it is more diffuse, and shifts primarily towards the phenyl ring on arm $B$, and onto N32 on Arm B. The singlet GS and ES structural data are compared in Table 2. The transition mainly appears to break the conjugation by placing more electron density from Arm B onto the central carbon and withdrawing electron density from the phenyl hydrogens and the nitrogen to compensate. This characterises itself as a form of charge transfer transition.

Table 2: Comparison of singlet ground and first excited state structural data

\begin{tabular}{|l|ll|}
\hline & Singlet GS & Singlet ES \\
\hline Arm Angle & & \\
Arm A & $33^{\circ}$ & $24 / 20^{\circ}$ \\
Arm B & $33^{\circ}$ & $93 / 87^{\circ}$ \\
Arm C & $33^{\circ}$ & $24 / 20^{\circ}$ \\
\hline C1-Ph length & & \\
Arm A & $1.44 \AA$ & $1.44 \AA$ \\
Arm B & $1.44 \AA$ & $1.50 \AA$ \\
Arm C & $1.44 \AA$ & $1.44 \AA$ \\
\hline Ph-N bond Length & & \\
Arm A & $1.36 \AA$ & $1.37 \AA$ \\
Arm B & $1.36 \AA$ & $1.36 \AA$ \\
Arm C & $1.36 \AA$ & $1.37 \AA$ \\
\hline Atomic Charge & & \\
C1 & 0.25 & -0.04 \\
C2 & -0.10 & 0.06 \\
C12 & -0.10 & -0.10 \\
C22 & -0.10 & 0.06 \\
N33 (Arm 1) & -0.40 & -0.40 \\
N32 (Arm 2) & -0.40 & -0.33 \\
N34 (Arm 3) & -0.40 & -0.40 \\
\hline
\end{tabular}




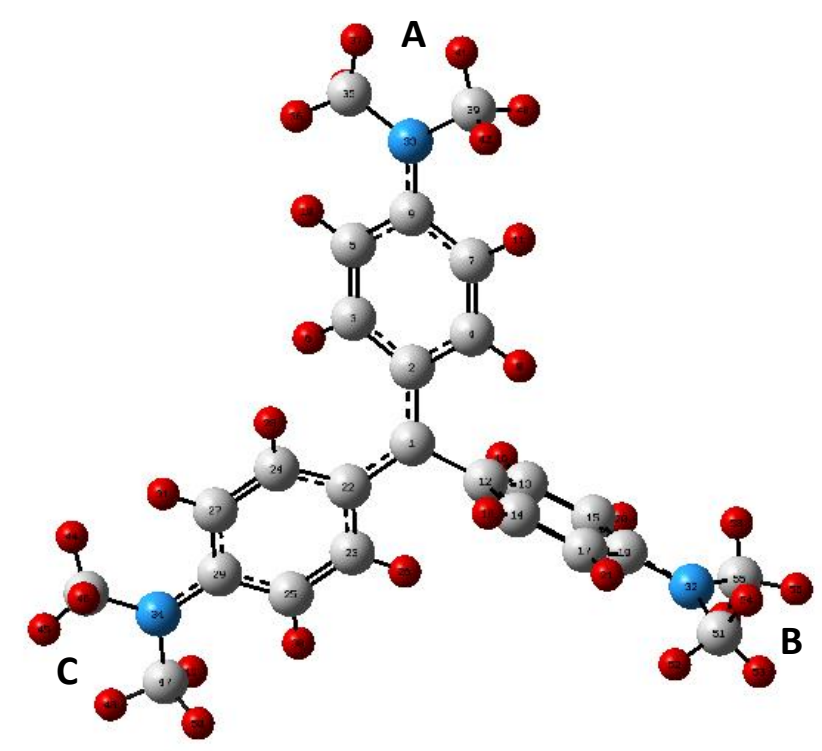

Figure 6: Optimised geometry of singlet first excited state of crystal violet

The vertical excitation energy between the singlet GS and the first excited state is $251 \mathrm{kJmol}^{-1}$ which corresponds to the predicted spectrum peak of $477 \mathrm{~nm}$, but after vibrational relaxation to the optimised excited state geometry this decreases by $87 \mathrm{kJmol}^{-1}$ to $164 \mathrm{kJmol}^{-1}$. This corresponds to a predicted emission at $731 \mathrm{~nm}$ as depicted in Figure $1 \mathrm{c}$.

The triplet first excited state configuration is generated by one of the beta electrons from the doubly occupied HOMO-2 orbital has moving into the HOMO-1 orbital which was previously singly occupied by an alpha electron (Figure 7). This is a minority spin transition between HOMO-2 and HOMO-1 (Figure 8). This leaves the molecule with HOMO and HOMO-2 singly occupied, retaining its triplet state.

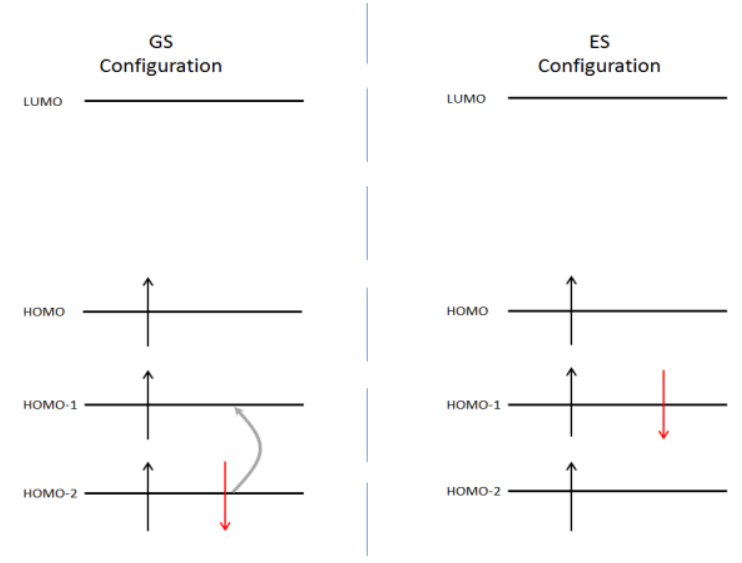

Figure 7: MO diagram of electronic transition for triplet CV first excited state 

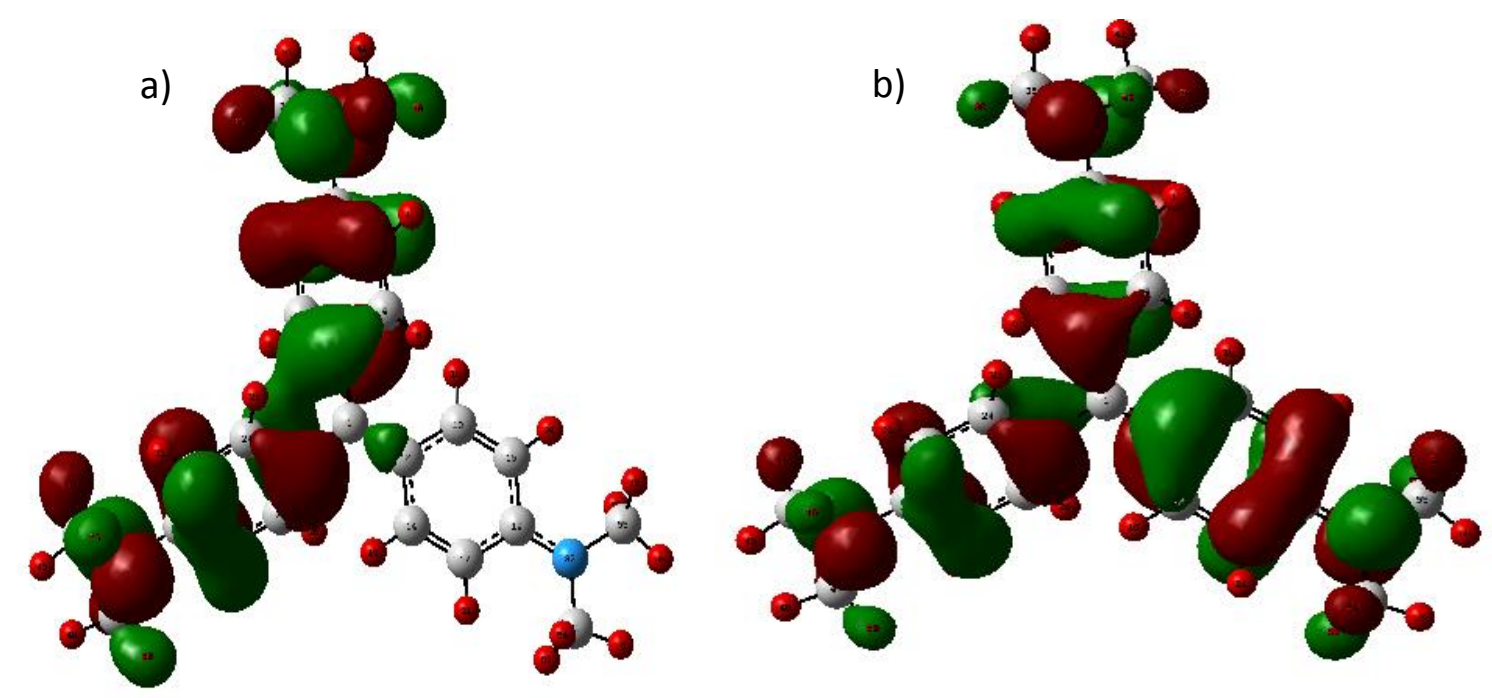

Figure 8: Minority spin MOs for CV lowest lying triplet state: a) HOMO-2 b) HOMO-1

In the HOMO-2 orbital it can be seen that there is a bonding interaction between the 'top' lobe on the central side of the phenyl group of Arm A and the 'bottom' lobe on the central side of the phenyl group on Arm C which circumvents the otherwise largely antibonding interaction between the two arms. This bonding mode arises from the angle of the arms and may be responsible for the distortion observed in these phenyl rings in the lowest lying triplet state. We can also see that there is little electron density on Arm B in HOMO-2, only a small sigma bonding mode between $\mathrm{C} 1$ and $\mathrm{C} 12$. In HOMO-1 we see that the MO looks essentially the same on Arm C, while Arm A is very similar but the phase has swapped. This means that the main interaction between $\operatorname{Arm} A$ and $\operatorname{Arm} C$ is no longer antibonding with a 'twisting' top to bottom bonding mode, but now it is top-top and bottom-bottom bonding and there is no twisting type of bonding interaction present. Arm B shows the largest change, in that it now resembles Arms A and C, but the in opposite phase. Therefore, the phenyl ring on Arm B is antibonding with respect to both other arms, which suggests that the geometric changes we can expect to come about as a result of the excited state electronic configuration are relaxation of the distortion of the phenyl rings on Arms $A$ and $C$, the $\mathrm{C} 1-\mathrm{C} 12$ bond should be elongated and the $\mathrm{C} 1-\mathrm{C} 2$ and $\mathrm{C} 1-\mathrm{C} 22$ bonds should match. We also expect to see a transfer of charge, with the phenyl ring and nitrogen on Arm B becoming more negatively charged.

The geometry of the triplet excited state was also calculated, though it was not possible to converge the forces completely, and the structure was found to be oscillating around a 
stationary point in the potential energy surface. Upon analysis of the total energies of these optimisation steps, it was found that the oscillation was occurring within an energy range of $<1 \mathrm{kJmol}^{-1}$. This was sufficient for the structure of lower energy to be considered as the minimum. The ES optimised structure has no symmetry, but is still approximately propeller shaped and actually resembles the singlet GS more than the triplet GS (Figure 9). The triplet GS and ES structural data are compared in Table 3.

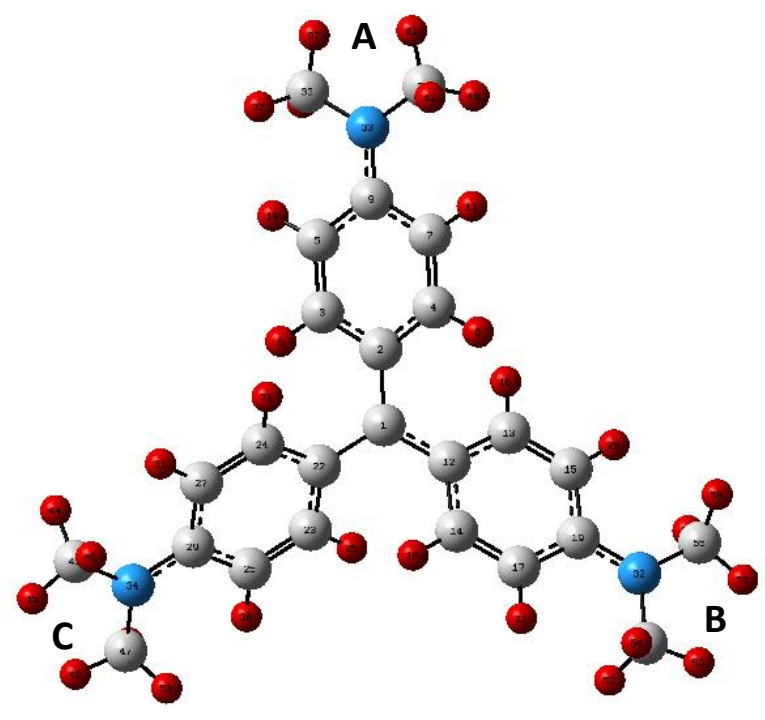

Figure 9: Optimised geometry of triplet first excited state of crystal violet

As predicted from the $\mathrm{MO}$ analysis, there is a slight lengthening of the $\mathrm{C} 1-\mathrm{C} 12$ bond and the $\mathrm{C} 1-\mathrm{C} 2$ and $\mathrm{C} 1-\mathrm{C} 22$ bonds are similar and slightly shorter. The C1-C2 bond is shortened more because there is a slight $\mathrm{C} 1-\mathrm{C} 2$ bonding interaction in the HOMO-1. The main difference between the triplet GS and ES geometries is that the angle between the arms and the molecular plane has altered to more closely match the singlet GS geometry, though it is still very far from being $C_{3}$ symmetric.

The vertical excitation energy between the triplet GS and first excited state is only 20.0 $\mathrm{kJmol}^{-1}$. The vibrational relaxation only lowers the energy by $0.9 \mathrm{kJmol}^{-1}$, so the energy difference between the triplet GS and ES is $19.1 \mathrm{kJmol}^{-1}$. 
Table 3: Comparison of triplet ground and first excited state structural data

\begin{tabular}{|c|c|c|}
\hline & Triplet GS & Triplet ES \\
\hline $\begin{array}{l}\text { Arm Angle } \\
\text { Arm A } \\
\text { Arm B } \\
\text { Arm C }\end{array}$ & $\begin{array}{l}44^{\circ} / 41^{\circ} \\
19^{\circ} \\
44^{\circ} / 41^{\circ}\end{array}$ & $\begin{array}{l}34 / 32^{\circ} \\
28 / 24^{\circ} \\
38 / 36^{\circ}\end{array}$ \\
\hline $\begin{array}{l}\text { C1-Ph length } \\
\text { Arm A } \\
\text { Arm B } \\
\text { Arm C } \\
\end{array}$ & $\begin{array}{l}1.47 \AA \\
1.43 \AA \\
1.47 \AA \\
\end{array}$ & $\begin{array}{l}1.46 \AA \\
1.44 \AA \\
1.46 \AA \\
\end{array}$ \\
\hline $\begin{array}{l}\text { Ph-N bond Length } \\
\text { Arm A } \\
\text { Arm B } \\
\text { Arm C }\end{array}$ & $\begin{array}{l}1.369 \AA \\
1.366 \AA \\
1.369 \AA\end{array}$ & $\begin{array}{l}1.37 \AA ̊ \\
1.36 \AA \\
1.37 \AA\end{array}$ \\
\hline
\end{tabular}




\section{Chromium(III) Compounds}

There are nine chromium(III) complexes which were used in this work, and each was examined in the quartet state, which is the expected ground state for $\mathrm{d}^{3}$ complexes, as well as the doublet state where computationally viable. The compounds are hexafluorochromate (Figure 10a), hexaaminochromate (Figure 10b), hexathiocyanatochromate (Figure 10c), hexaureachromium (Figure 10d), chromium acetylacetonate (Figure 10e), hexacyanochromate (Figure 10f), hexaimidazolidone chromium (Figure 10g), trioxalatochromate (Figure 10h) and tris(ethylenediamine) chromium (Figure 10i). The results for each compound will be presented and discussed separately.

a)
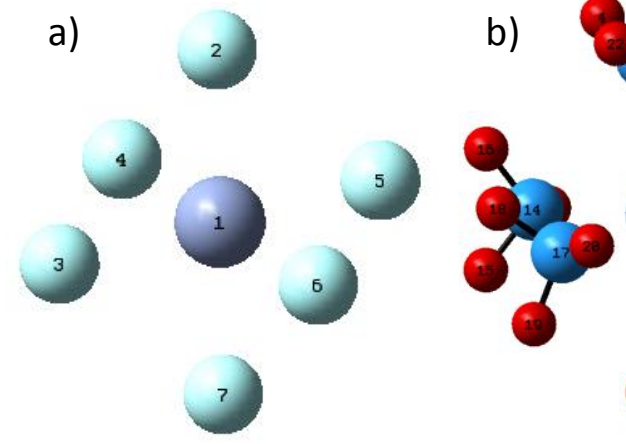

b)
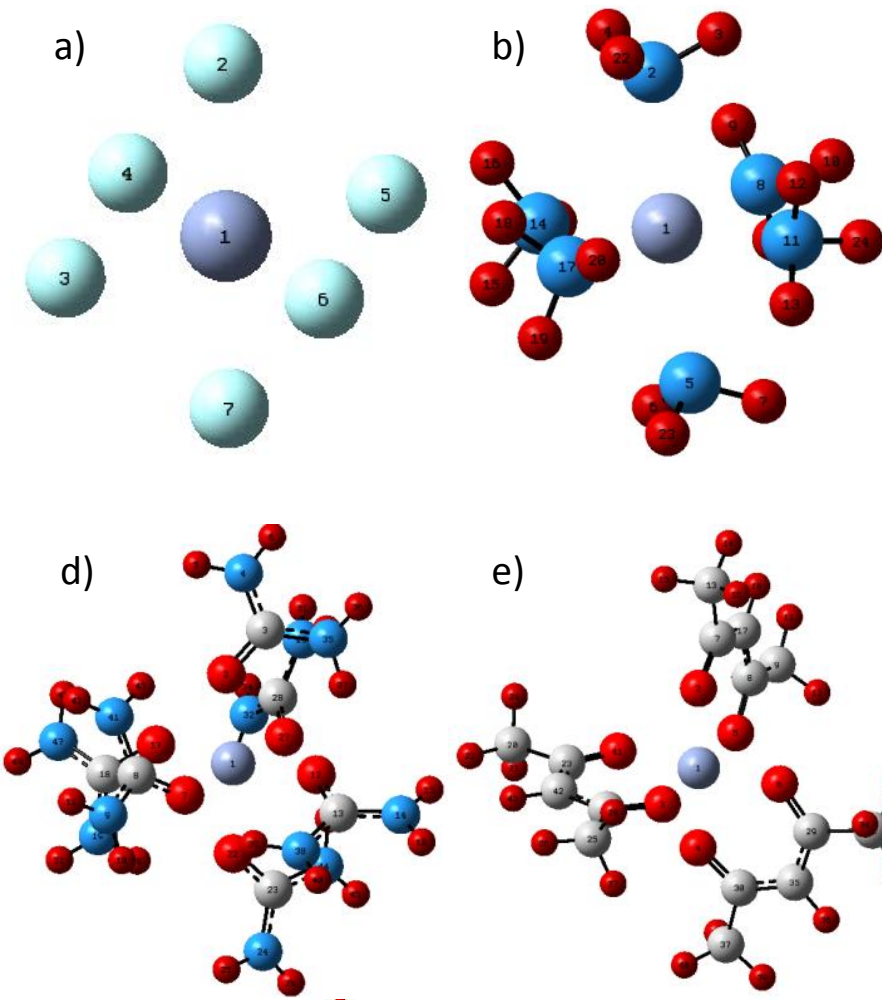

g)

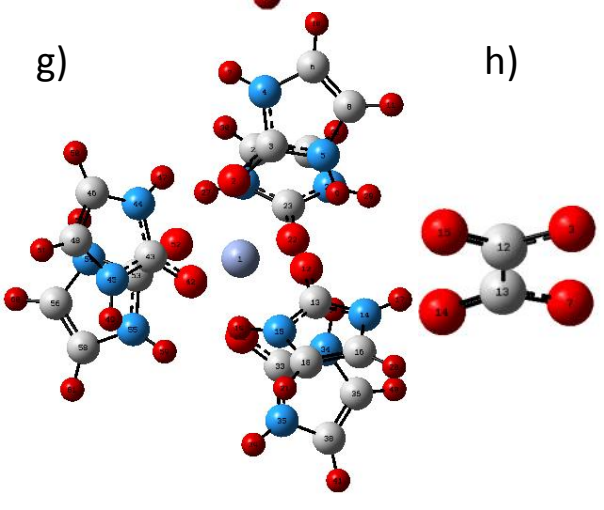

e)

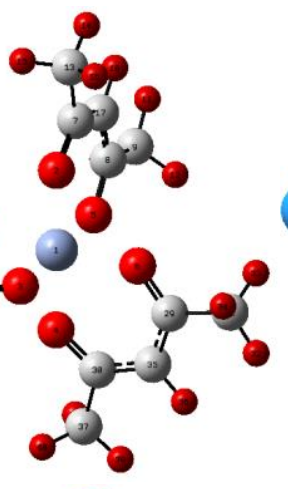

c)

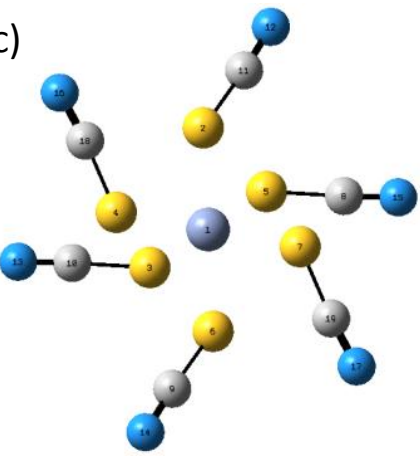

f)
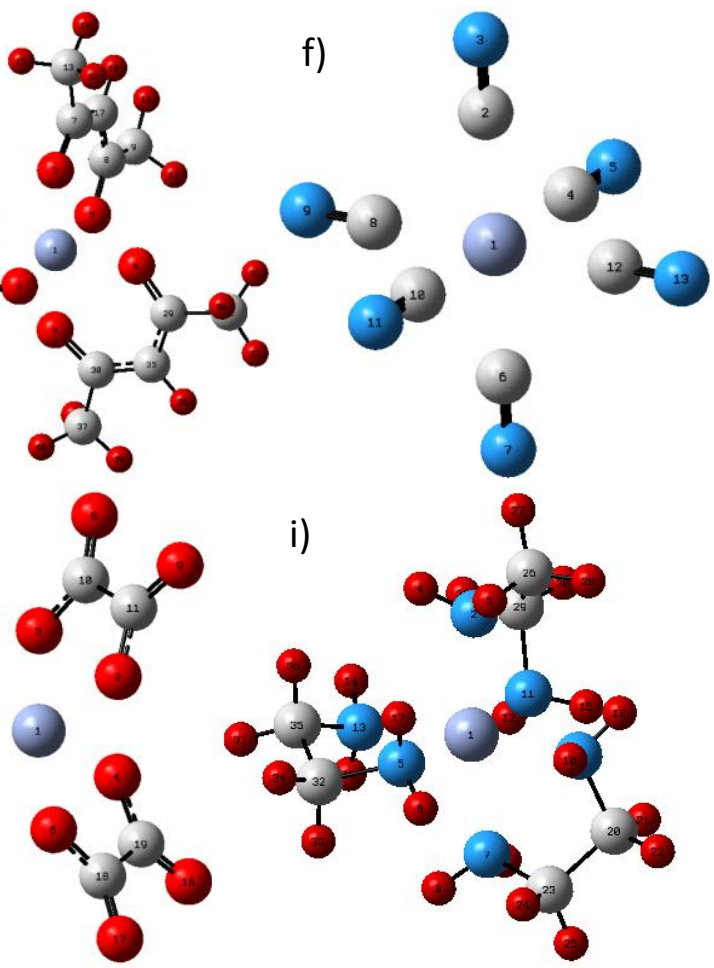

Figure 10: Optimised ground state structures for the examined nine chromium compounds (Grey=C, $C y a n=F, B l u e=N$, Yellow=S, Large Red=0, Small red=H) 
In many cases the experimental spectra are obtained in the visible region only and with very low epsilon values which are due to forbidden transitions. These are, in some cases, not visible in our predicted spectra as the presence of allowed predicted peaks at higher energy makes them lie below the curve. For X3LYP the mean absolute deviation of the error (MAE) is $+0.22 \mathrm{eV}[38]$ so the method is expected to overestimate the energy.

\section{Hexafluorochromate $\left[\mathrm{CrF}_{6}\right]^{3-}$}

The quartet ground state of hexafluorochromate has $O_{h}$ symmetry (Figure 11 ), with a $\mathrm{Cr}-\mathrm{F}$ distance of $1.98 \AA$. The Mulliken charge on the metal is 1.74 while the fluorine ligands each carry a charge of -0.79 , as expected from the highly electronegative fluorine.

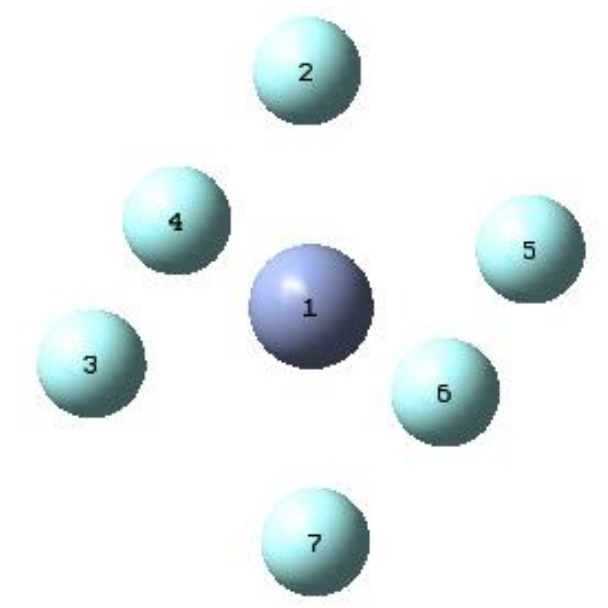

Cr-F distance of $1.97 \AA$. This state is close to being octahedral, only slight bond angle deviations from $90^{\circ}$

Figure 11: Hexafluorochromate distorts it. The Mulliken charges are $\mathrm{Cr}=1.73$ and $F=-0.79$.

The allowed electronic transitions are high in energy, as all the lower energy transitions in the usual UV-Vis range are symmetry forbidden. As a result, although the first 60 excited states were calculated there are only two allowed peaks in the absorbance spectrum of the quartet state, at $200 \mathrm{~nm}$ with an $\varepsilon$ of 2500 and at $181 \mathrm{~nm}$ with $\varepsilon$ of 3100 (Spectrum 2a). The doublet state also has 2 peaks in the absorbance spectrum at $198 \mathrm{~nm}$ with $\varepsilon$ of 2700 and at $176 \mathrm{~nm}$ with $\varepsilon$ of 1500 (Spectrum 2b). The first excited states of both the quartet and the doublet are symmetry forbidden and occur at low energy. The quartet ES is at $591 \mathrm{~nm}$ and the doublet ES is at $3370 \mathrm{~nm}$. This matches closely the theoretical results of Pierloot and Vanquickenborne of the first quartet transition at 598nm, though this was obtained at the $\mathrm{Cl}(3 \mathrm{~d})$ level of theory. At higher levels of theory such as $\mathrm{Cl}(3 \mathrm{~s}+3 p+3 \mathrm{~d})$ it was found to be at $684 \mathrm{~nm}$ [45]. Various hexafluorochromate salts have been synthesised and characterised, however there is little reliable literature on the absorption spectrum of this molecule. The Schlaefer et al paper from 1967 shows some results of absorption by $\left[\left(\mathrm{NH}_{3}\right)_{3}\left(\mathrm{CrF}_{6}\right)\right]$. This 
data shows peaks at $638 \mathrm{~nm}, 439 \mathrm{~nm}$ and $284 \mathrm{~nm}$, with extremely low $\varepsilon$ [46]. These peaks were not found on the predicted spectrum.

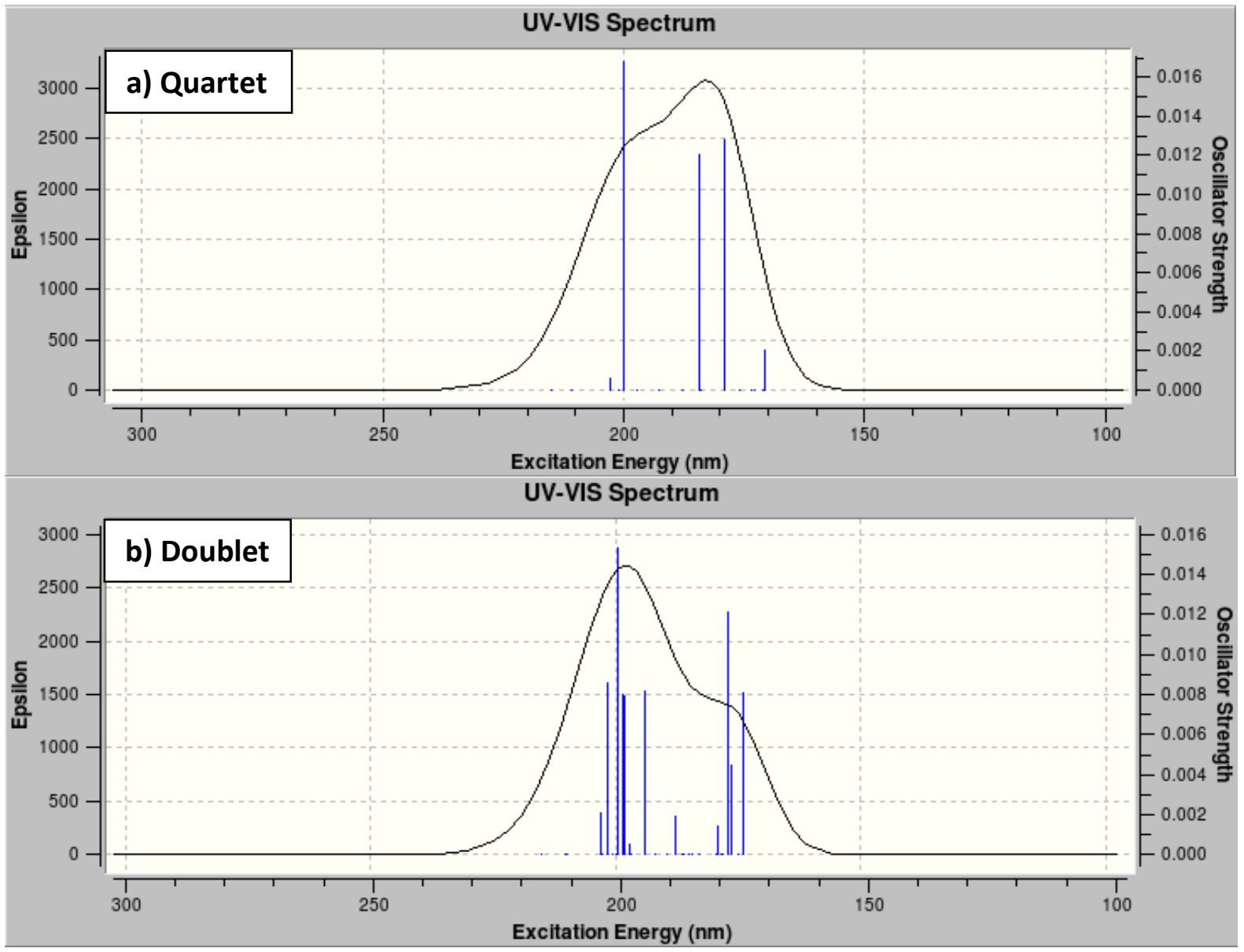

Spectrum 2: UV-Vis spectra of hexafluorochromate

The quartet first excited state configuration arises from two main electronic transitions from the $T_{2 g}$ HOMO to the two degenerate $E_{g}$ molecular orbitals which comprise the LUMO. The first configuration contributes $71 \%$ of the excited state while the second contributes $19 \%$. Visual determination of the nature of the molecular orbitals shows that HOMO is antibonding between the $d_{x y}$ orbital on the metal and the $p$ orbitals on the ligands in the $x y$ plane (Figure 12b), one MO in the LUMO is antibonding between the orbital on the ligand and the $p$ orbitals on the ligands in the $x y$ plane and weakly antibonding to the ligands on the z-axis, and the other MO in the LUMO is antibonding between the metal and the $\mathrm{p}$ orbitals on all 6 ligands, though less strongly to the x-axis ligands (Figure 12a). 
a) LUMO

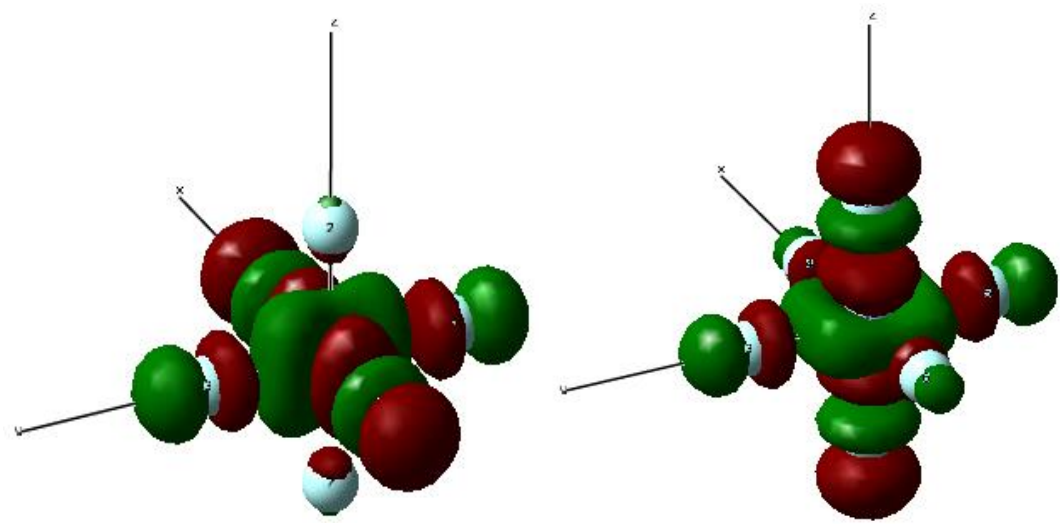

b) HOMO

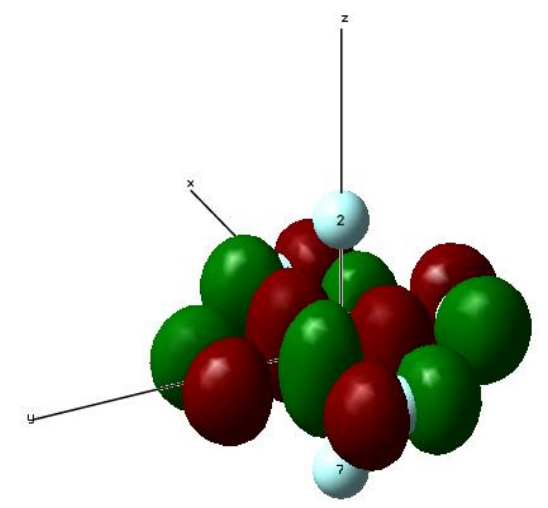

Figure 12: Relevant ground state MOs of $\left[\mathrm{CrF}_{6}\right]^{3-}$ quartet: a) LUMO b) HOMO

This suggests an increase in the antibonding character between the metal and the z-axis ligands in the first excited state, and a slight decrease in the antibonding character between the metal and the $x$-axis ligands while the ligands on the $y$-axis will remain relatively unaffected.

The geometry of the quartet first excited state was calculated and was found to have $D_{2 h}$ symmetry. The $\mathrm{Cr}$ - $\mathrm{F}$ bond lengths were $2.03 \AA$ along the z-axis, $2.10 \AA$ along the $\mathrm{x}$-axis and $1.95 \AA$ along the $y$-axis.

The vertical excitation energy between the quartet ground state and first excited state was found to be $202 \mathrm{kJmol}^{-1}$. There is no appreciable decrease in energy due to vibrational relaxation so it is clear that the transition has excited the molecule into the lowest vibrational state of the first excited state already. Consequently, upon optimisation we find that there are only miniscule geometric differences between the ground state and the excited state. 
The orbital contributions to the doublet first excited state are complex. There are a number of electronic configurations, but the largest contributors to the excited state configuration are the HOMO-11 $\alpha\left(A_{g}\right) \rightarrow L U M O+1 \alpha\left(A_{g}\right)$, the HOMO $\alpha\left(A_{g}\right) \rightarrow L U M O+1 \alpha\left(A_{g}\right)$, the HOMO-13 $\beta\left(A_{g}\right) \rightarrow L U M O+1 \beta\left(A_{g}\right.$, and the $\operatorname{HOMO} \beta\left(A_{g}\right) \rightarrow L U M O+1 \beta\left(A_{g}\right)$. A visual determination of the nature of the MOs shows that the HOMO-11 is sigma bonding between the metal and all ligands except F3 and F5 which are non-bonding (Figure 13c). The HOMO $\alpha$ is pi antibonding between the metal and all ligands (Figure 13b), and the LUMO+1 $\alpha$ is pi antibonding between the metal and all ligands except F3 and F5 which are non-bonding (Figure 13a). The HOMO-13 $\beta$ is sigma bonding between the s-orbital on the metal and $\mathrm{p}$ orbitals on all the ligands (Figure 13d). The HOMO $\beta$ is pi antibonding between the metal and all ligands except F3 and F5 which are non-bonding (Figure 13b). The LUMO+1 $\beta$ is pi antibonding to all the ligands (Figure 13a). The HOMO-11 $\alpha\left(A_{g}\right) \rightarrow L U M O+1 \alpha\left(A_{g}\right)$ transition will have an effect on the structure as the bonding modes changes from a sigma type bonding to a pi type antibonding. The $\mathrm{HOMO} \alpha\left(A_{g}\right) \rightarrow L U M O+1 \alpha\left(A_{g}\right)$ transition will result in a decrease in antibonding character between the metal and F3 and F5 so the $\mathrm{Cr}-\mathrm{F}$ bond distance will increase for these ligands. The HOMO-13ß $\left(A_{g}\right) \rightarrow L U M O+1 \beta\left(A_{g}\right)$ transition will have a large effect on the excited state structure as the interaction between the metal and ligands changes from bonding to antibonding for all ligands, which should have the effect of increasing the $\mathrm{Cr}-\mathrm{F}$ distance for all ligands. The $\mathrm{HOMO}\left(\mathrm{A}_{\mathrm{g}}\right) \rightarrow \mathrm{LUMO}+1 \beta\left(\mathrm{A}_{\mathrm{g}}\right)$ transition will have the opposite effect to the $\operatorname{HOMO}\left(A_{g}\right) \rightarrow L U M O+1 \beta\left(A_{g}\right)$ transition and so will mitigate the effect of that transition. The main overall effect that can be seen is that there is a slight electron density shift in all transitions towards the metal from the ligands. This suggests a ligand to metal charge transfer transition. Structurally, the excited state configuration should cause the equatorial ligands to move further away from the metal, with F3 and F5 moving slightly closer. 
a) LUMO+1 $\alpha$
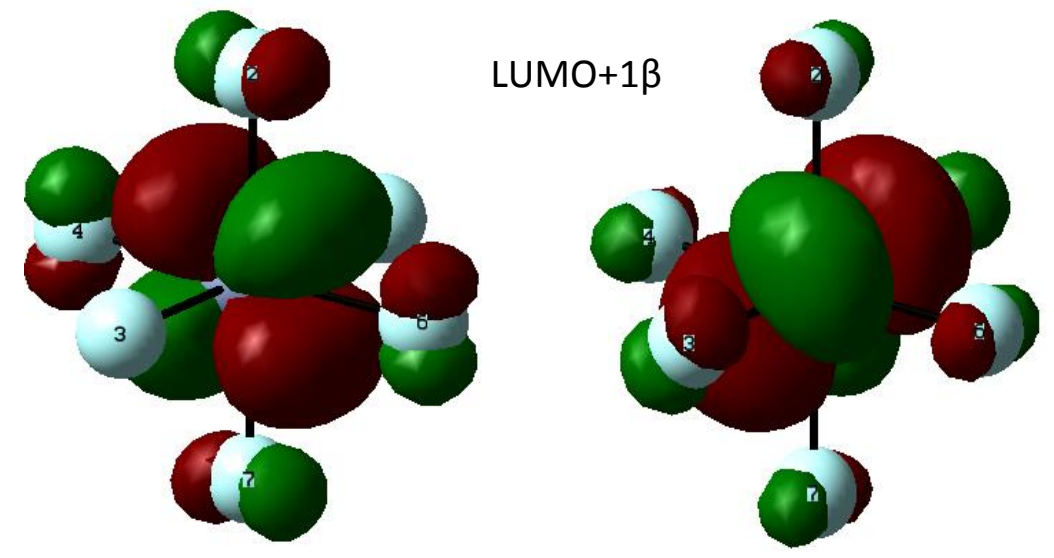

b) $\mathrm{HOMO \alpha}$
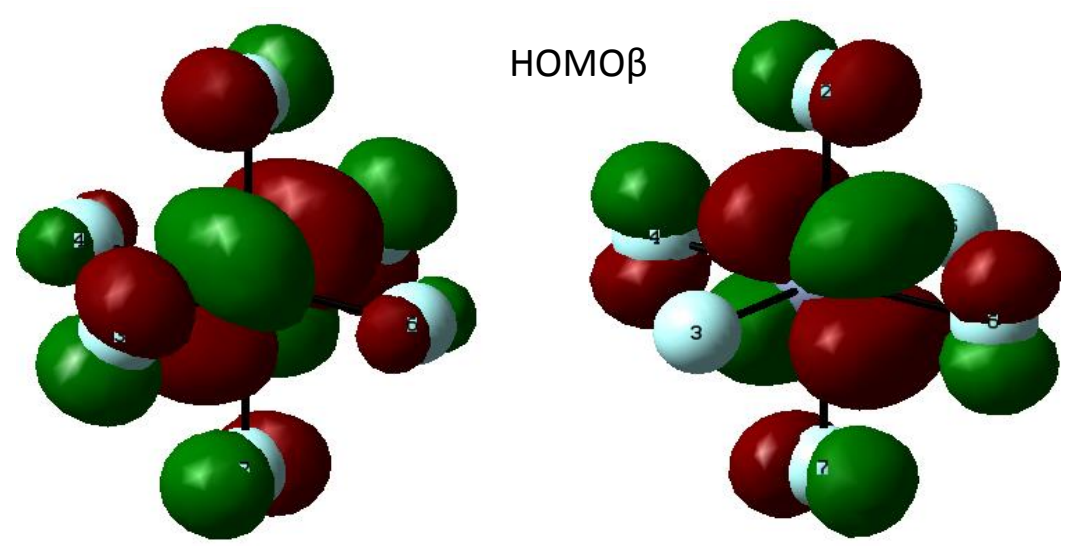

c) HOMO-11 $\alpha$

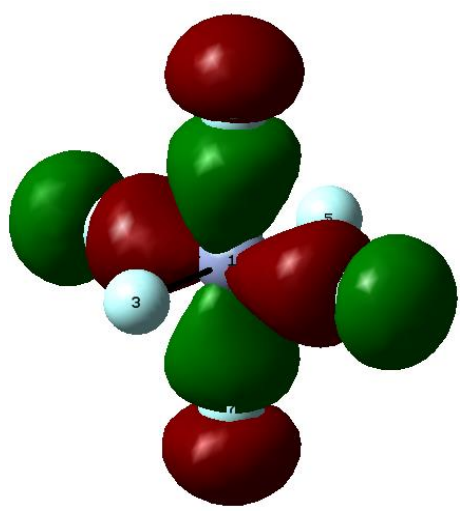

d) HOMO-13 $\beta$

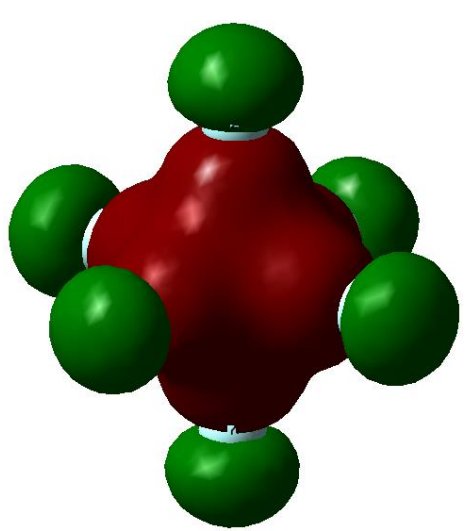

Figure 13: Relevant ground state MOs of $\left[\mathrm{CrF}_{6}\right]^{3-}$ doublet: a) LUMO+1 b) HOMO c) HOMO-11 $\alpha$ d) HOMO-13ß 
The geometry of the doublet first excited state was calculated and was found to have $D_{4 h}$ symmetry. The "axial" Cr-F distance is $1.96 \AA$ while the "equatorial" Cr-F distances are $1.98 \AA$. The Mulliken charges are $\mathrm{Cr}=1.72, \mathrm{~F} 2 / \mathrm{F} 7=-0.78$, other $\mathrm{F}=-0.79$ so only a minor change in charge position is observed. This is similar to the prediction, where ligands on one axis move closer to the metal slightly and the other two sets of ligands move further away. We also see a slight decrease of 0.01 in the positive charge on the metal

The vertical excitation energy between the doublet lowest lying and first excited state was found to be $36 \mathrm{kJmol}^{-1}$, and vibrational relaxation of the excited state only lowered the energy by a small amount $\left(1 \mathrm{kJmol}^{-1}\right)$ due to the minimal geometric changes.

A benchmark study was also performed on the quartet state of this molecule. This study compared the calculated vertical excitation energy of the molecule as determined by the X3LYP method to a benchmark CIS-D calculation using a number of different basis sets, namely 6-311G(d), CC-pVTZ, CC-pVQZ and CC-pV5Z. The results are collected in Table 4 and show that for the $6-311 \mathrm{G}(\mathrm{d})$ basis set used in this experiment the error is $+14.5 \%$ compared to the CISD calculation. Use of a larger coupled-cluster basis set can reduce this error considerably, however there is an increased computational cost associated with more comprehensive basis sets.

Table 4: Comparison of different levels of theory on first excited state absorbance energy

\begin{tabular}{|c|c|c|c|c|c|c|}
\hline Basis set & X3LYP (nm) & X3LYP (kJmol-1) & $\mathrm{CISD}(\mathrm{nm})$ & CISD (kJmol-1) & $\Delta \mathrm{E}(\mathrm{kJmol}-1)$ & $\%$ error \\
\hline $6-311 G(d)$ & 590.93 & 202.44 & 691.05 & 173.11 & 29.33 & 14.5 \\
\hline cc-pVTZ & 602.59 & 198.52 & 657.94 & 181.82 & 16.70 & 8.4 \\
\hline cc-pVQZ & 610.56 & 195.93 & 650.05 & 184.03 & 11.90 & 6.1 \\
\hline$c c-p V 5 Z$ & 613.16 & 195.10 & --- & --- & --- & --- \\
\hline
\end{tabular}




\section{Hexaaminochromate $\left[\mathrm{Cr}\left(\mathrm{NH}_{3}\right)_{6}\right]^{3+}$}

The quartet ground state of hexaaminochromate has $C_{2}$ symmetry, which bisects the $\mathrm{N} 2-\mathrm{Cr}-\mathrm{N} 14$ angle (Figure 14). The $\mathrm{Cr}-\mathrm{N}$ distances are the same at $2.15 \AA$, and the $\mathrm{N}-\mathrm{Cr}-\mathrm{N}$ angles are distorted slightly from $90^{\circ}$ which is the main source of the $C_{2}$ symmetry. The Mulliken charge on the metal is 1.69 while the nitrogens each carry a charge of -1.10 and the hydrogens each carry around 0.44 .

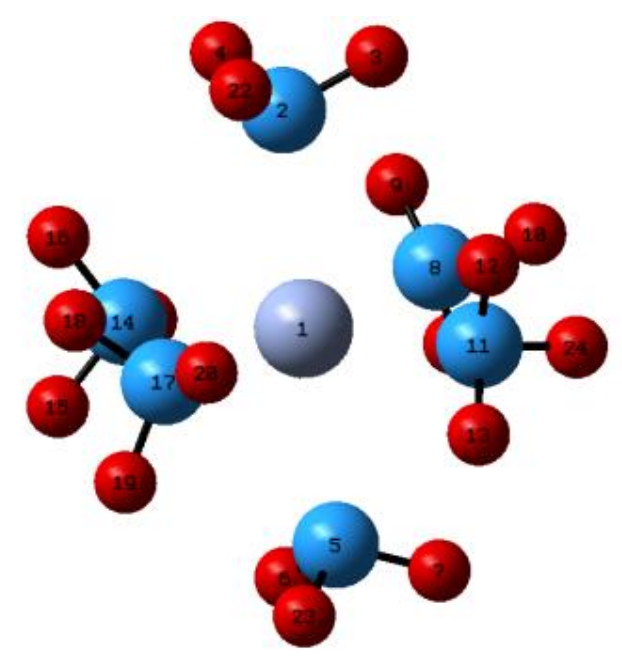

Figure 14: Hexaaminochromate

For the lowest lying doublet state, the $\mathrm{Cr}-\mathrm{N}$ bonds are all $2.14 \AA$ and the $\mathrm{N}-\mathrm{Cr}-\mathrm{N}$ neighbouring bond angles are all within a few degrees of $90^{\circ}$. This molecule therefore has $C_{3}$ symmetry. The Mulliken charges are $\mathrm{Cr}=1.68, \mathrm{~N}=-1.10$ and $\mathrm{H}=\mathrm{about} 0.44$.

The quartet has electronic transitions which are in the visible range but have epsilon values of less than 10 , corresponding to forbidden transitions, so there are 3 observed peaks in the UV region, which are at $198 \mathrm{~nm}$ with an $\varepsilon$ of $12500,151 \mathrm{~nm}$ with $\varepsilon$ of 40000 , and $133 \mathrm{~nm}$ with $\varepsilon$ of 10000 (Spectrum 3a).

The doublet also has electronic transitions in the very low energy region (around $3300 \mathrm{~nm}$ ) as well as in the visible region, which are symmetry forbidden and so it has two visible peaks in the UV region at $193 \mathrm{~nm}$ with $\varepsilon$ of 4000 and at $158 \mathrm{~nm}$ with $\varepsilon$ of 48000 (Spectrum 3b). This set of spectra shows an interesting feature as the two are very similar except for the small peak in the quartet spectrum at $133 \mathrm{~nm}$ which does not occur at all in the doublet spectrum. 


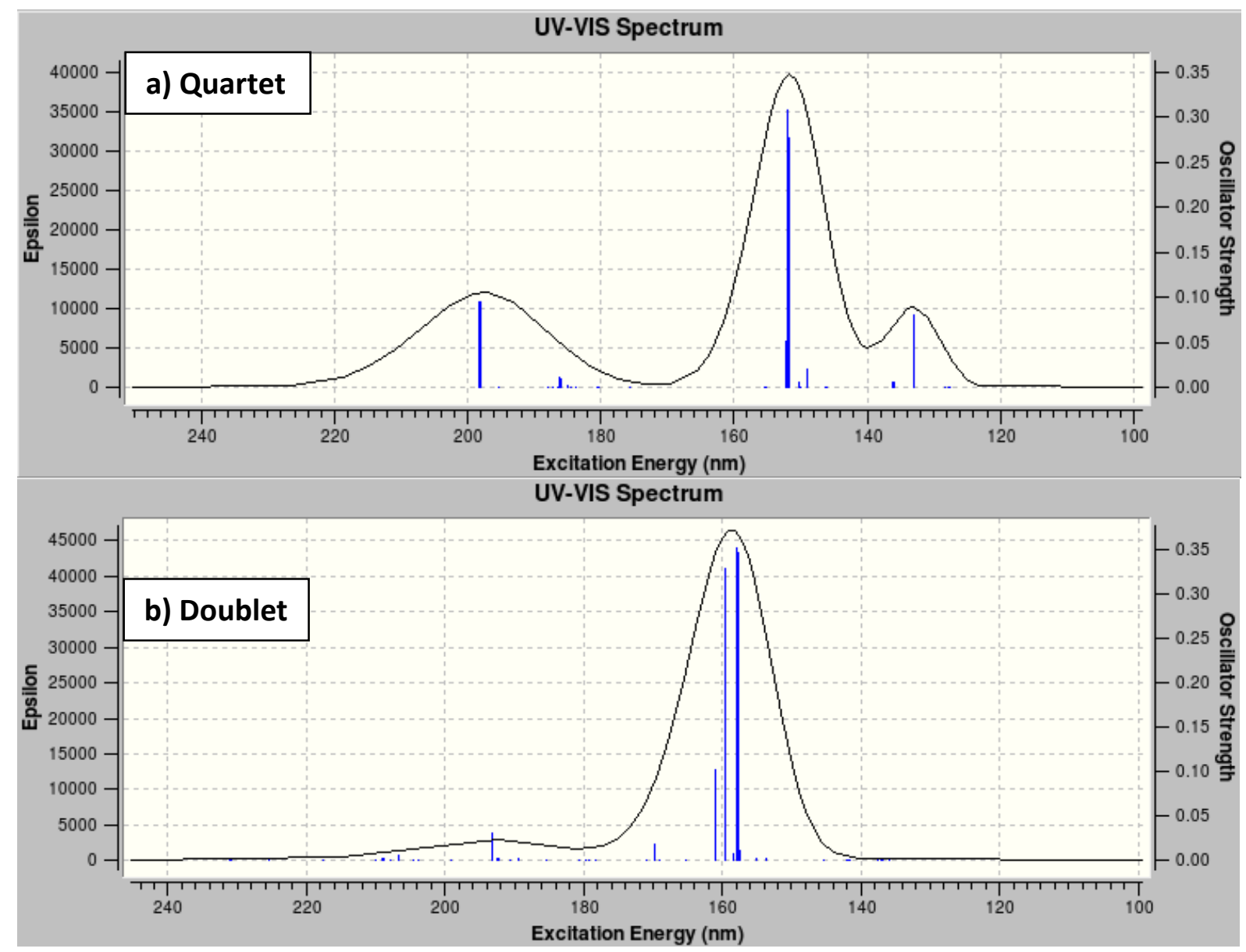

Spectrum 3: UV/Vis spectrum for hexaaminochromate

The quartet first excited state configuration arises from two main electronic transitions from the two degenerate MOs which comprise the HOMO to the two degenerate MOs which comprise the LUMO (Figure 15). Visual determination of the nature of the molecular orbitals shows that one MO in the HOMO has a $d_{y z}$ type orbital on the metal, which is slightly bonding towards the p-orbital on N8 and N17. The metal is weakly antibonding with respect to the other 4 ligands, and each ligand shows some $\mathrm{N}-\mathrm{H}$ bonding to either 1 or 3 of the $\mathrm{H}$. The other $\mathrm{MO}$ in the $\mathrm{HOMO}$ is non-bonding between the metal and the N8 and N17 ligands, and weakly bonding with respect to the other 4, again with some $\mathrm{N}-\mathrm{H}$ bonding (Figure 15b). One $\mathrm{MO}$ in the LUMO is non-bonding between the metal and ligands N8 and N17, and antibonding with respect to all other ligands. The other MO in the LUMO is antibonding between the metal and all ligands, but slightly more strongly towards N8 and N17 (Figure 15a). 
a) LUMO

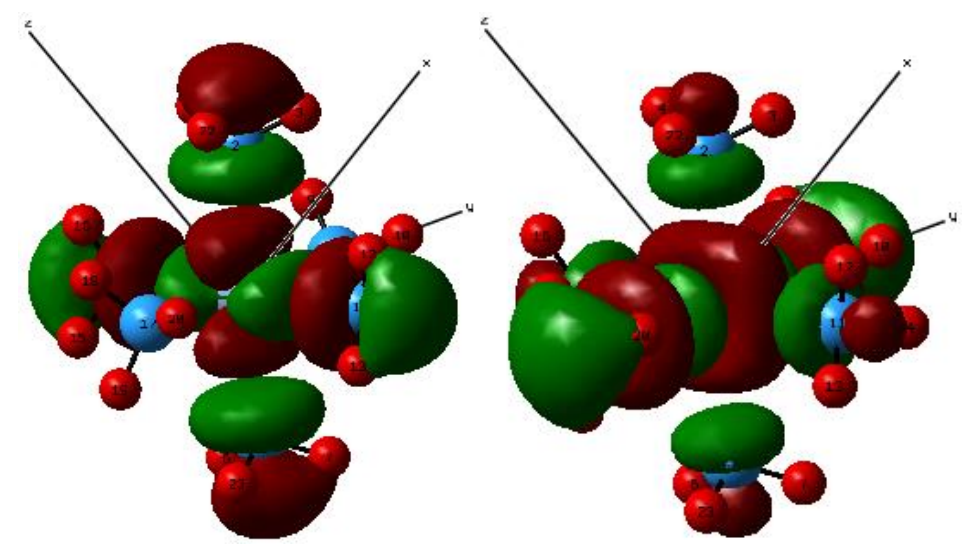

b) HOMO
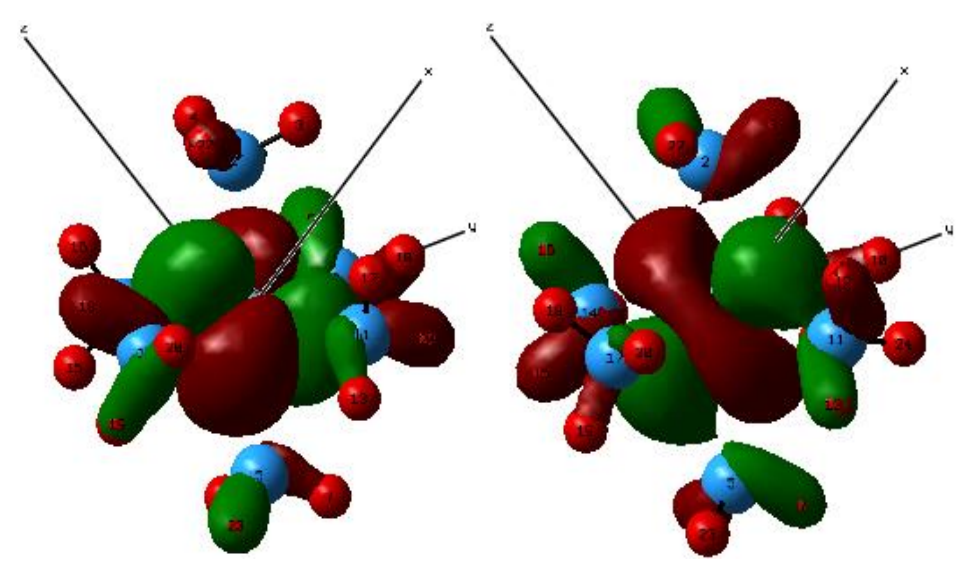

Figure 15: Relevant ground state MOs of $\left[\mathrm{Cr}\left(\mathrm{NH}_{3}\right)_{6}\right]^{3+}$ quartet: a) LUMO b) HOMO

The major ES configuration (65\%) shows that N8 and N17 lose all their MO density while there is a shift of density onto the other four ligands. The change in interaction from the HOMO, being weakly bonding between the metal orbital and small transverse p-orbitals on the $\mathrm{N}$, to the LUMO, being strongly antibonding between the metal $\mathrm{d}_{\mathrm{xz}}$ orbital and the $\mathrm{xz}-$ plane ligands' parallel p-orbitals, will distort the molecule, whereby the y-axis ligands are closer to the metal than the others. The minor ES configuration (29\%) will have a similar effect, but in this case will be more intense with respect to the $y$-axis ligands than the others, mitigating the effect of the major configuration somewhat. Also, as the slight bonding interactions observed in the HOMO were between the metal orbital and one side of the p-orbital on the nitrogen only, the release of this constraining bonding interaction may cause greater distortion of the angles in the molecule than observed in the GS.

The geometry of the quartet first excited state was calculated and it was found to retain its $C_{2}$ symmetry along the same axis. The metal to ligand distances changed to $\mathrm{Cr}-\mathrm{N} 2 / \mathrm{N} 14=2.21$ $\AA$, $\mathrm{Cr}-\mathrm{N} 8 / \mathrm{N} 17=2.12 \AA$ and $\mathrm{Cr}-\mathrm{N} 5 / \mathrm{N} 11=2.35 \AA$. As expected, we have seen an increase in $\mathrm{Cr}-\mathrm{N}$ bond lengths for all ligands but N8 and N17, which have shortened slightly. The angles 
between ligands in the GS were all within a few degrees of $90^{\circ}$, but in the excited state this is significantly distorted as was predicted above, with $\mathrm{N} 2-\mathrm{Cr}-\mathrm{N} 14=122^{\circ}$ and $\mathrm{N} 5-\mathrm{Cr}-\mathrm{N} 11=74^{\circ}$ (Figure 16). This distortion exaggerates the $C_{2}$ symmetry of the molecule by increasing the $\mathrm{N}-\mathrm{Cr}-\mathrm{N}$ angle by a considerable amount in a $C_{2}$ symmetrical manner. The Mulliken charges were $\mathrm{Cr}=1.74, \mathrm{~N} 2 / \mathrm{N} 14=-1.12, \mathrm{~N} 8 / \mathrm{N} 17=-1.10, \mathrm{~N} 11 / \mathrm{N} 5=-1.10, \mathrm{H}$ range between 0.42-0.45. This shows that there is some movement of charge density from the metal to the ligands suggesting a metal-ligand charge transfer transition.

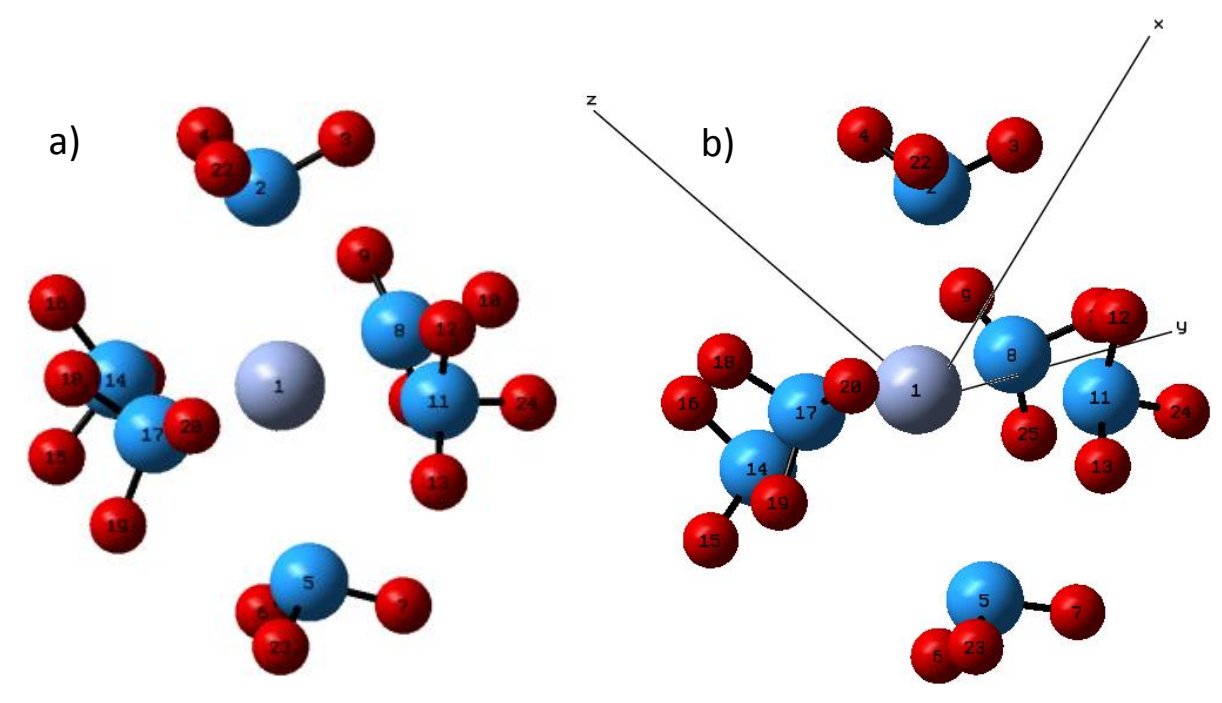

Figure 16: Optimised geometries of quartet hexaaminochromate: a) GS b) ES

The vertical excitation energy of the quartet was found to be $266 \mathrm{kJmol}^{-1}$, however due to the structural differences between the GS and ES, vibrational relaxation lowers the energy by $33 \mathrm{kJmol}^{-1}$, leaving the difference between quartet ground and first excited states as 233 $\mathrm{kJmol}^{-1}$. This corresponds to a predicted emission of $515 \mathrm{~nm}$. The vertical excitation energy for doublet hexaaminochromate was found to be $36 \mathrm{kJmol}^{-1}$. 


\section{Hexathiocyanatochromate $\left[\operatorname{Cr}(\mathrm{SCN})_{6}\right]^{3-}$}

The quartet ground state of hexathiocyanatochromate has $S_{6}$ symmetry (Figure 17 ). The molecule has $\mathrm{Cr}$-S distance of $2.51 \AA$, S-C distance of $1.69 \AA$ and C-N distance of $1.17 \AA$. The S-Cr-S angles are $97^{\circ}$ and $83^{\circ}$, so the molecule is distorted from an octahedral positioning of the $\mathrm{S}$ atoms. The ligands sit at a $114^{\circ} \mathrm{Cr}-\mathrm{S}-\mathrm{C}$ angle and are slightly bent with a $174^{\circ}$ S-C-N angle. The Mulliken charges are $\mathrm{Cr}=1.21, \mathrm{~S}=-0.30, \mathrm{C}=-0.03$ and $\mathrm{N}=-0.38$.

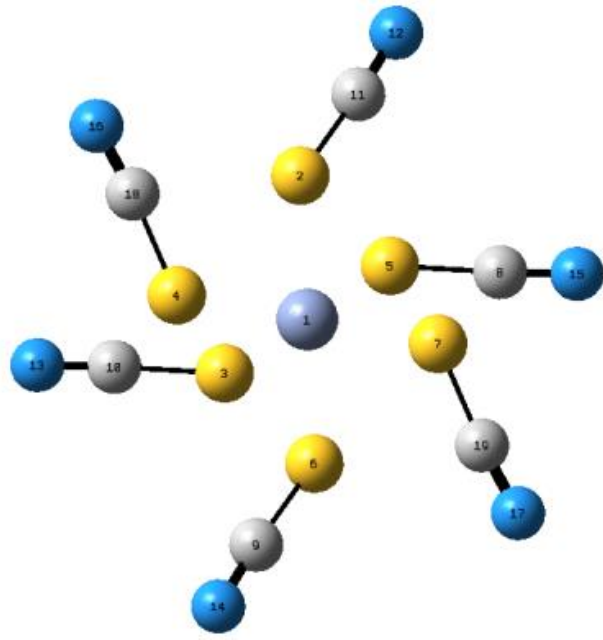

Figure 17: Hexathiocyanatochromate The quartet has symmetry forbidden electronic transitions in the low energy end of the visible region and two peaks are visible in the UV/Vis spectrum at $390 \mathrm{~nm}$ with an $\varepsilon$ value of 12000 , and at $300 \mathrm{~nm}$ with an $\varepsilon$ value of 9000 . The peaks are comprised of a number of electronic transitions which are close together in energy (Spectrum 4). There are also transitions which are hidden by the larger peaks, but are significant enough to note. These can be seen on the left of the first peak at about $459 \mathrm{~nm}$ with an $\varepsilon$ of 5500 (Spectrum 4). The first excited state is at lower energy at $739 \mathrm{~nm}$ but is symmetry forbidden. The quartet hexathiocyanate is expected to be coloured red/yellow as it absorbs in the blue/violet region.

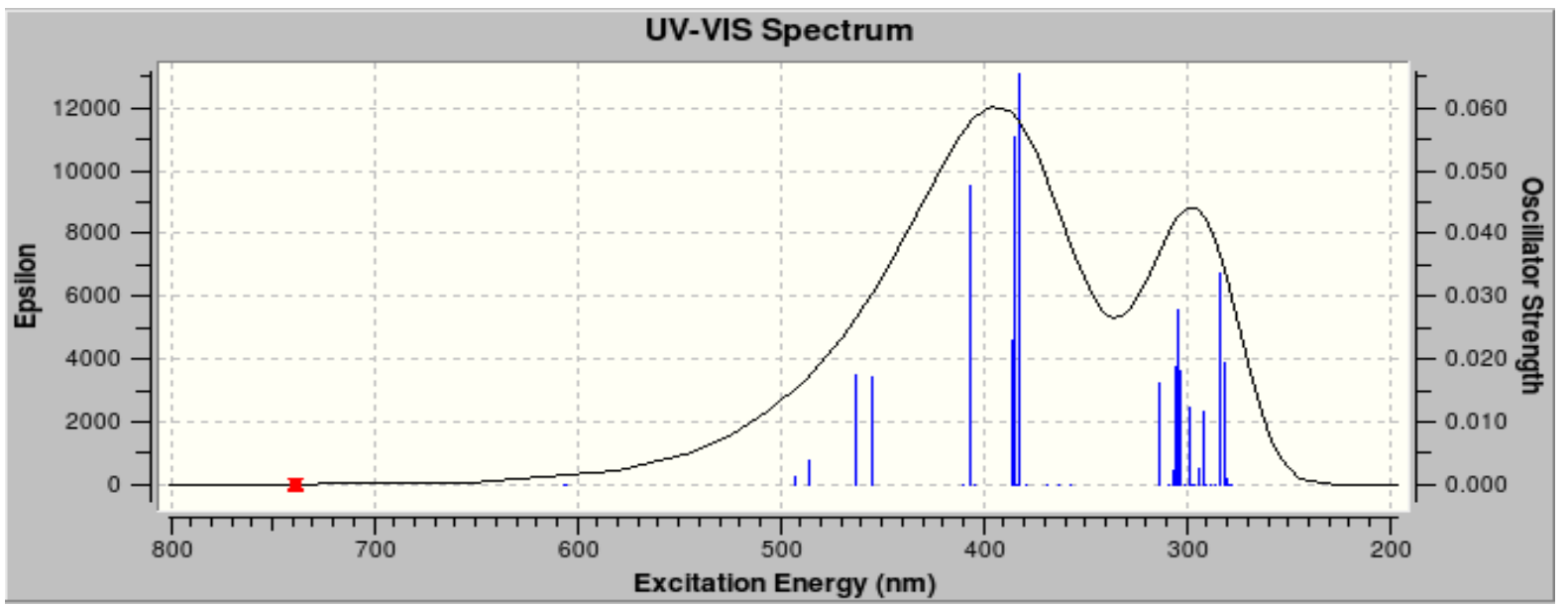

Spectrum 4: UV/Vis spectrum of quartet hexathiocyanatochromate

Experimental data for hexathiocyanatochromate in aqueous solution shows peaks at 564 $\mathrm{nm}, 419 \mathrm{~nm}, 309 \mathrm{~nm}, 234 \mathrm{~nm}$ and $218 \mathrm{~nm}$ [47], with the three highest energy peaks being attributed to charge transfer bands which are clearly higher energy than the 60 states which 
were calculated. When the spectrum is recalculated in aqueous solution, the peaks shift to $401 \mathrm{~nm}$ and $294 \mathrm{~nm}$. When these are adjusted for the $0.22 \mathrm{eV}$ error the peaks should actually be at $432 \mathrm{~nm}$ and $322 \mathrm{~nm}$. This is closer but clearly still quite far off the experimental data. However, as there are transitions with low epsilon values lying below the theoretical curve it is possible that the experimental data actually related to one of these. There are transitions visible in aqueous solution at $483 \mathrm{~nm}, 477 \mathrm{~nm}$ and $453 \mathrm{~nm}$. These adjust for error to $528 \mathrm{~nm}, 521 \mathrm{~nm}$ and $492 \mathrm{~nm}$. If these are taken into account the picture looks better, with a good correlation between the experimental peaks at $564 \mathrm{~nm}, 419 \mathrm{~nm}$ and $309 \mathrm{~nm}$, and the adjusted theoretical peaks at around $525 \mathrm{~nm}, 432 \mathrm{~nm}$ and $322 \mathrm{~nm}$. The other possibility is that the experimental data is for thiocyanate bonded through the $\mathrm{N}$, rather than the $\mathrm{S}$. This bonding type was not explored in this work due primarily to time constraints.

The first excited state configuration arises from two main electronic configurations which contribute $84 \%$ of the state between them. They are $\mathrm{HOMO}-8\left(E_{g}\right) \rightarrow$ LUMO(Eg $(32 \%)$ and $\mathrm{HOMO}\left(E_{g}\right) \rightarrow L U M O\left(E_{g}\right)(52 \%)$ with two transitions occurring from each of the HOMO-8 and the HOMO. Visual determination of the nature of the MOs shows that one of the degenerate MOs in the HOMO-8 is weakly bonding between the metal and S2 and S6, but essentially non-bonding with respect to the other ligands. Also all the ligands show a C-N bonding interaction between $\mathrm{p}$ orbitals. The other $\mathrm{MO}$ in HOMO-8 shows non-bonding interactions between the metal and S2 and S6, but is bonding with respect to the other ligands. All ligands show the same $\mathrm{C}-\mathrm{N}$ bonding as in the other MO (Figure 18c). One of the MOs in the HOMO shows mainly antibonding interaction between the metal and the ligands but there is some bonding interaction between the ligands directly through the sulfur $p$ orbitals. The S-C interaction shows an antibonding mode. Also, most of the electron density is on the ligands. The other $\mathrm{MO}$ in the HOMO is antibonding between the metal and ligands and also shows bonding interaction between sulphur atoms through their p-orbitals (Figure 18b). One of the MOs in the LUMO is antibonding between the metal and S3, S4, S5, and S7 and non-bonding with respect to S2 and S6. It also shows some bonding interaction between $\mathrm{S}$ and $\mathrm{C}$ and antibonding between $\mathrm{C}$ and $\mathrm{N}$. The other $\mathrm{MO}$ in the LUMO is antibonding between the metal and all ligands, but more strongly towards S2 and S6, and only weakly towards S4 and S7. There is a bonding interaction between S and C and an 
antibonding interaction between $\mathrm{C}$ and $\mathrm{N}$ (Figure 18a). The transitions from the first degenerate pair (HOMO-8) (32\%) both result in a shift in electron density from being more concentrated on the metal to being spread more evenly across the molecule, and also shows a change in nature as the metal to ligand interaction goes from bonding to antibonding and the intra-ligand bonding shifts from mostly $\mathrm{C}-\mathrm{N}$ to a stronger $\mathrm{S}-\mathrm{C}$ bond. The transitions from the second degenerate pair (HOMO) (52\%) are expected to have a larger effect. The bonding interaction between the p-orbitals on the sulfur atoms is lost, which may destroy the $S_{6}$ symmetry as it is reasonable that this bonding interaction is responsible for the unusual angle at which the ligands sit in the GS. We also see that the antibonding interaction between the metal and the ligands is generally retained in the ES configuration, except that in the LUMO the antibonding interaction is a sigma rather than pi interaction.

a) LUMO
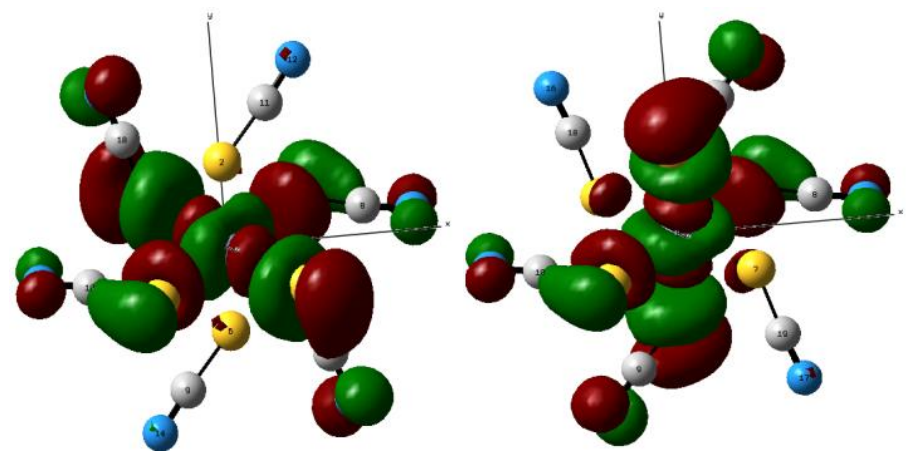

b) HOMO
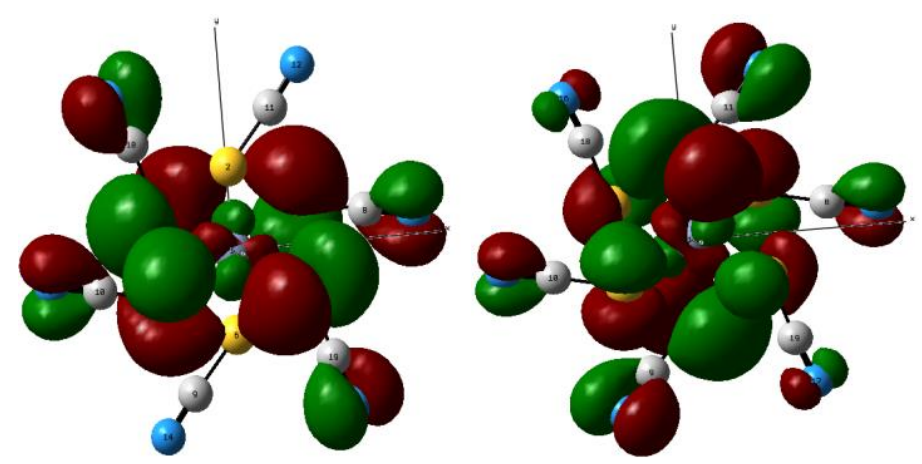

c) $\mathrm{HOMO}-8$
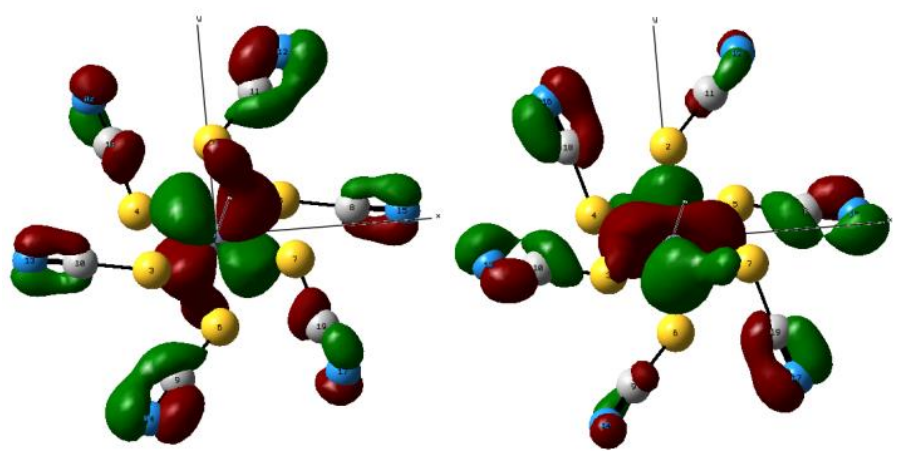

Figure 18: Relevant ground state MOs for $\left[\mathrm{Cr}(\mathrm{SCN})_{6}\right]^{3-}$ quartet: a) LUMO b) HOMO-8 c) HOMO 
The geometry of the quartet excited state was also calculated, though it was not possible to converge the forces completely, and the calculation was found to be oscillating around a stationary point in the potential energy surface. Upon analysis of the total energies of the relevant optimisation steps, it was found that the oscillation was occurring within an energy range of $<3 \mathrm{kJmol}^{-1}$. This was sufficient for the structure of lower energy to be considered as the minimum. Also, a calculation of the vibrational frequencies showed that there were no negative vibrational modes in this structure. The structure was found to have no symmetry, although it is very close to being of $C_{i}$ symmetry. It has a $\mathrm{Cr}$-S2/S5 distance of $2.80 \AA$, CrS3/S5 distance of $2.45 \AA$ and a Cr-S4/S7 distance of $2.50 \AA$. The S2-C11 bond length is $1.70 \AA$, S3-C10 bond length is $1.70 \AA$ and the S4-C18 bond length is $1.69 \AA$. The C-N bond lengths are all $1.16 \AA$. The ligands sit at a $125^{\circ}$ angle for ligands $C 2 / C 6,116^{\circ}$ angle for ligands $C 3 / C 5$ and a $117^{\circ}$ angle for ligands $\mathrm{C} 4 / \mathrm{C} 7$. They are all slightly bent with a $173^{\circ} \mathrm{S}-\mathrm{C}-\mathrm{N}$ angle (Figure 19 ).

a)

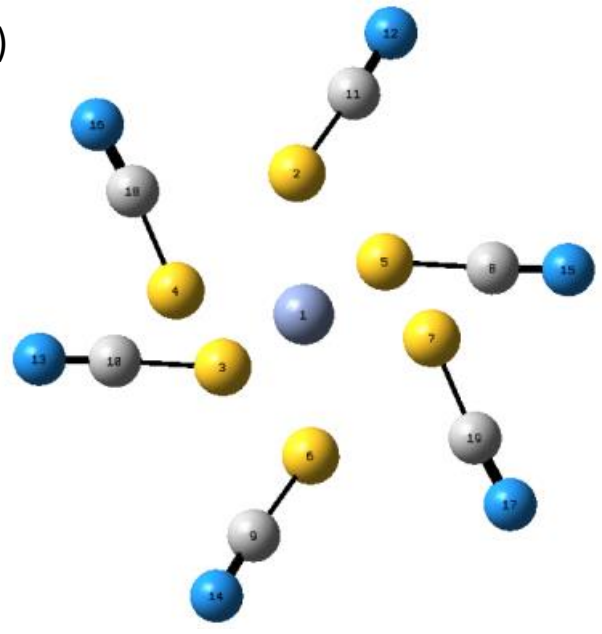

b)

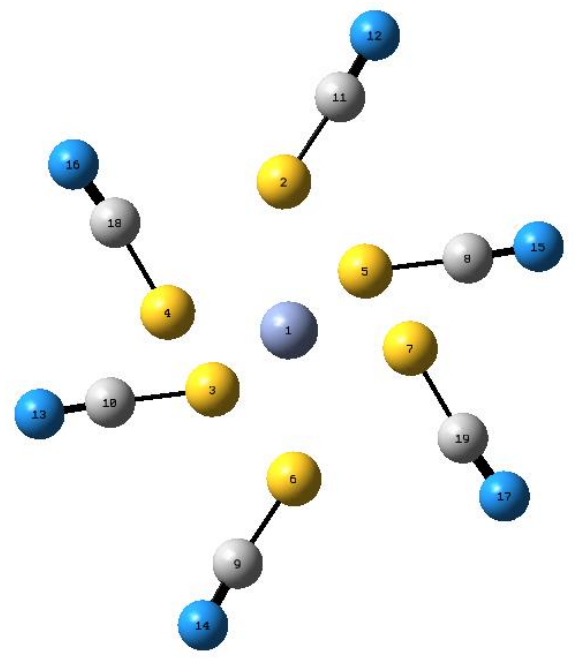

Figure 19: Optimised geometries of quartet hexthiocyanatochromate: a) GS b) ES

The vertical excitation energy for quartet hexathiocyanatochromate was found to be $162 \mathrm{kJmol}^{-1}$. Vibrational relaxation lowered the energy by $26 \mathrm{kJmol}^{-1}$ which means that the energy difference between the GS and ES is $136 \mathrm{kJmol}^{-1}$. The lowest lying doublet state of hexathiocyanatochromate was unable to be obtained as it also reached an oscillation in the optimisation steps which was too large to consider being near the minimum. 


\section{Hexaureachromate $\left[\mathrm{Cr}\left(\mathrm{CONH}_{2}\right)_{2}\right]^{3+}$}

The quartet ground state of hexaureachromate has no discernable symmetry (Figure 20). The $\mathrm{Cr}$-O bond distance varies from $1.97 \AA$ to $2.02 \AA$, and the $\mathrm{C}=\mathrm{O}$ bonds vary from $1.27 \AA$ to $1.28 \AA$. The $\mathrm{C}-\mathrm{N}$ conjugated bonds vary from $1.33 \AA$ to $1.35 \AA$. The $\mathrm{Cr}-\mathrm{O}-\mathrm{C}$ bond angle is between $135^{\circ}$ and $138^{\circ}$. The Mulliken charge on $\mathrm{Cr}$ is 1.68, and the charge on the other atoms varies.

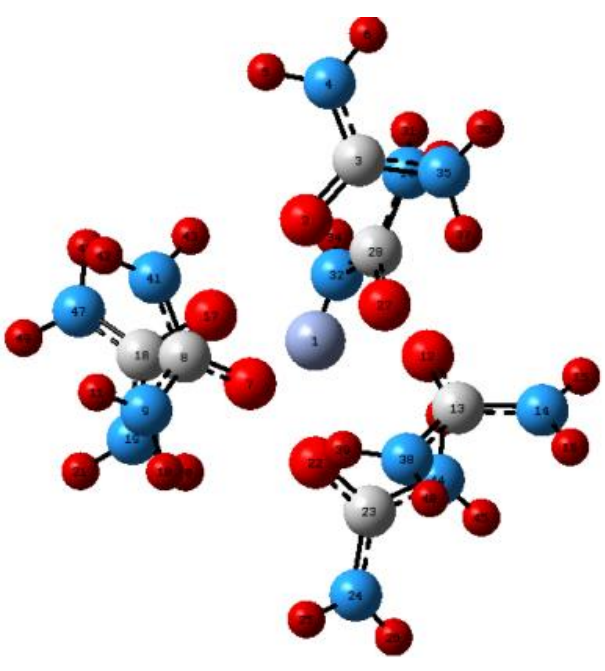
$\mathrm{O}=-0.60$ to $-0.64, \mathrm{C}=0.80$ to 0.84 and $\mathrm{N}=-0.77$ to -0.82 .

The doublet lowest lying state also has no symmetry.

Figure 20: Hexaureachromate The $\mathrm{Cr}-\mathrm{O}$ bond distance varies from $1.96 \AA$ to $2.01 \AA$, and the $\mathrm{C}=\mathrm{O}$ bonds vary from $1.27 \AA$ to $1.28 \AA$. The $\mathrm{C}-\mathrm{N}$ conjugated bonds vary from $1.33 \AA$ to $1.35 \AA$. The $\mathrm{Cr}-\mathrm{O}-\mathrm{C}$ bond angle is between $135^{\circ}$ and $139^{\circ}$. There is almost no difference between the quartet and doublet ground states in terms of geometrical disparity. The Mulliken charge on $\mathrm{Cr}$ is 1.66 and the charge on the other atoms varies. $\mathrm{O}=-0.59$ to $-0.64, \mathrm{C}=0.80$ to 0.84 and $\mathrm{N}=-0.77$ to -0.82 , so little difference of charge density is observed compared to the quartet state.

The predicted UV-Vis spectrum for quartet hexaureachromate is unusual as it contains a number of very weak transitions, as can be seen in the lower energy region at $558 \mathrm{~nm}$, which appear with $\varepsilon$ values less than 1000 . The main peak is at $230 \mathrm{~nm}$ and has an $\varepsilon$ of 6000 . This peak is made up of a large number of transitions within a relatively small range of energies as can be seen in Spectrum 5a. The first excited state appears at $603 \mathrm{~nm}$ but has a very low $\varepsilon$ value. The UV/Vis spectrum of the doublet state is similar in appearance to the quartet state, again having a few lower energy transitions with non-zero but low $\varepsilon$ values in the $420-580 \mathrm{~nm}$ range. The main peak is at $245 \mathrm{~nm}$ with $\varepsilon 6000$. It is also made up of a large number of transitions within a relatively small range of energies, and so the peak appears nonsymmetrical (Spectrum 5b). The first excited state appears at $3396 \mathrm{~nm}$ and so is very low energy, but has a very low $\varepsilon$ value. 


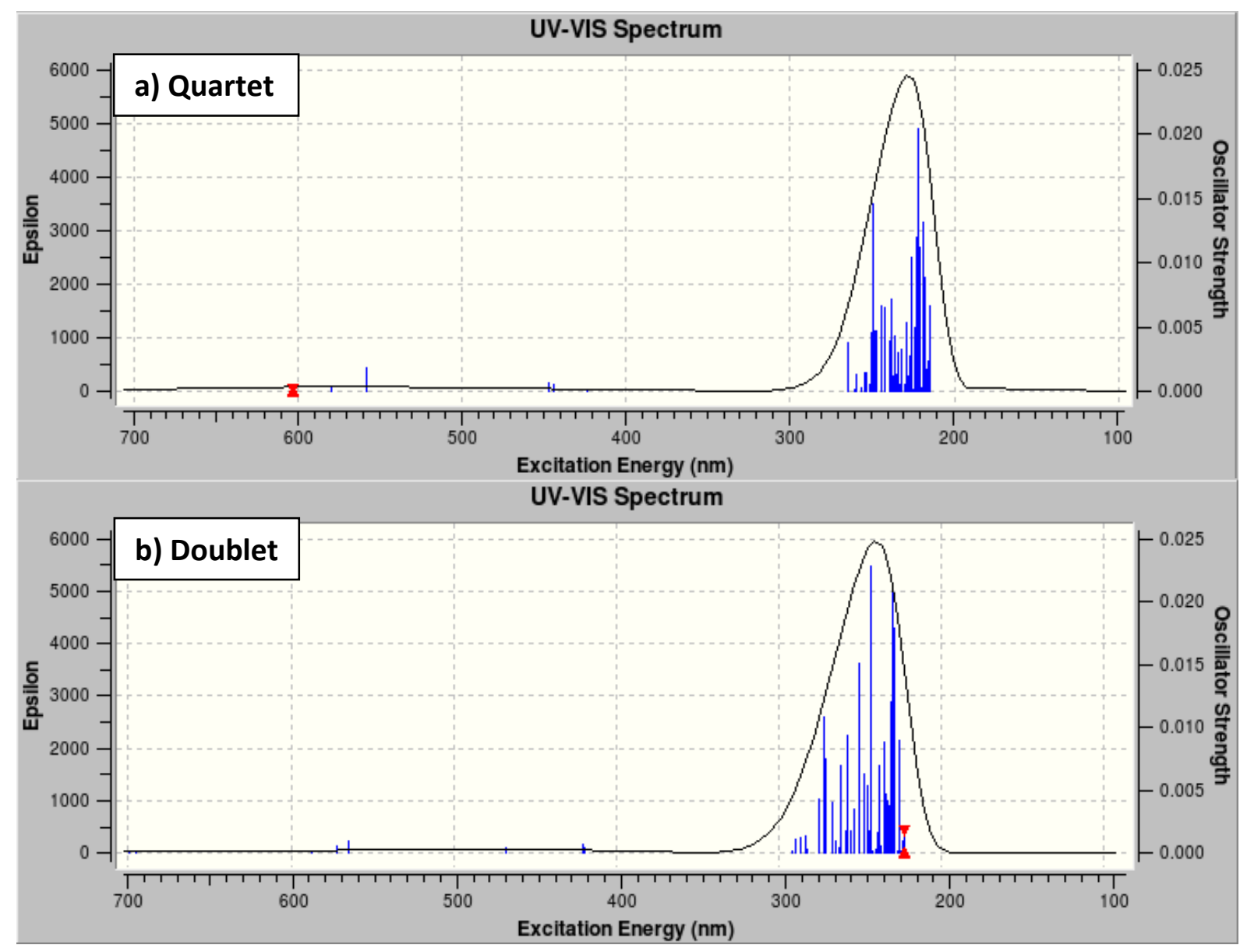

Spectrum 5: UV/Vis spectrum hexaureachromate

The experimental data for hexaureachromate in aqueous solution shows peaks at $700 \mathrm{~nm}$, $625 \mathrm{~nm}, 444 \mathrm{~nm}$ and $289 \mathrm{~nm}$ but with extinction coefficients below 75 [33]. This appears completely different to the predicted spectrum until you zoom in on the visible region at which point you can see small peaks (Spectrum 6). These small peaks have low epsilon values and so are not clearly visible in Spectrum 5, but transitions can be seen at $603 \mathrm{~nm}$, $580 \mathrm{~nm}, 557 \mathrm{~nm}, 444 \mathrm{~nm}$ and $423 \mathrm{~nm}$. When adjusted for the $0.22 \mathrm{eV}$ error, these transitions shift to $708 \mathrm{~nm}, 646 \mathrm{~nm}, 618 \mathrm{~nm}, 482 \mathrm{~nm}$ and $458 \mathrm{~nm}$. This is a much closer general match as the first three transitions form one peak and the second two form a second peak. The $700 \mathrm{~nm}$ peak observed in experiment was present as a shoulder on the $625 \mathrm{~nm}$ peak similar to what is observed here. Also, the experimental peak observed at 289 $\mathrm{nm}$ may be present in the prediction but hidden under the main peak. Remodelling in aqueous solution caused negligible change to the position of the transitions. 


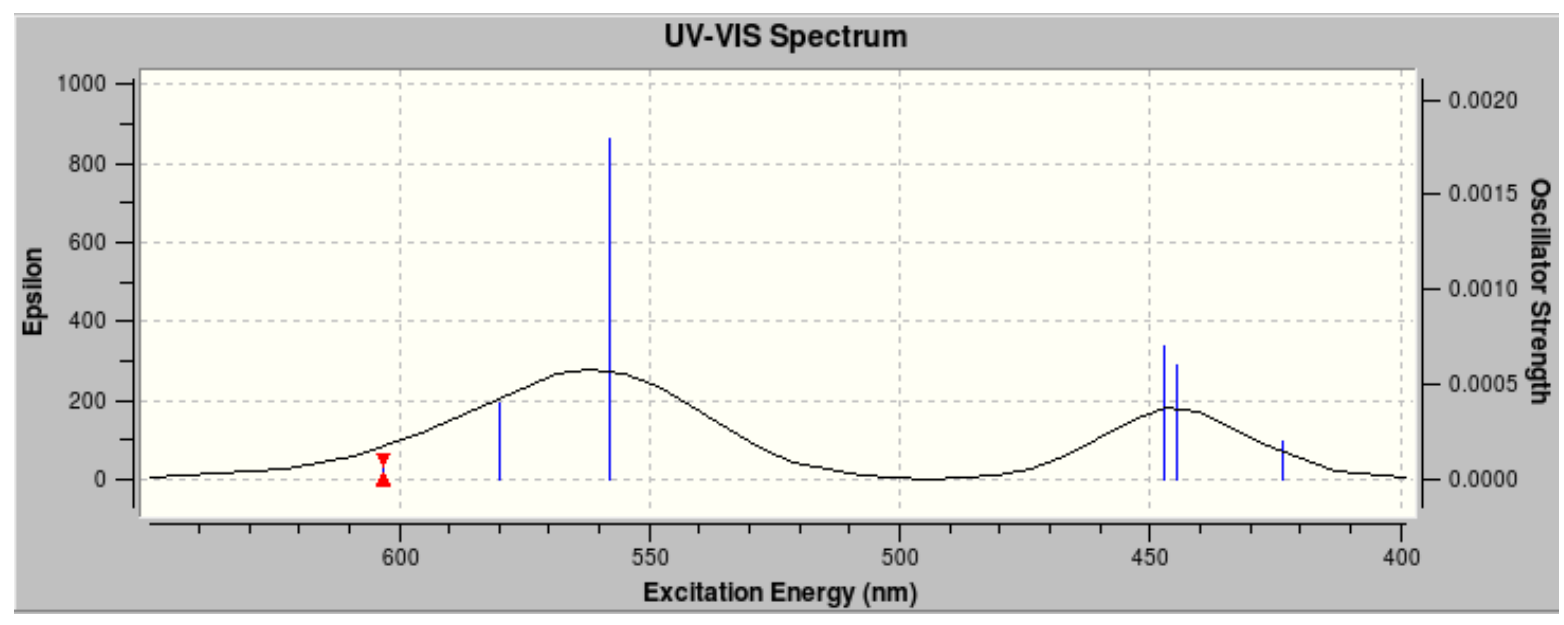

Spectrum 6: Zoom in on visible region of quartet absorbance spectrum of hexaureachromate

The quartet first excited state arises from 13 individual excited configurations, which means that our simple model of an excited state arising from a single excited determinant truly breaks down. To simplify the situation, only the 4 configurations which contribute the most towards the excited state configuration are considered. These are HOMO-20 $\rightarrow$ LUMO (11\%), HOMO-7 $\rightarrow$ LUMO (19\%), HOMO-6 $\rightarrow$ LUMO (9\%) and HOMO $\rightarrow$ LUMO (31\%) so the largest single contribution to the first excited state configuration comes from the HOMO->LUMO transition. Visual determination of the nature of the MOs shows that HOMO-20 is bonding between metal and all ligands except for 017, which is non-interacting. HOMO-20 also contains significant $\mathrm{O}-\mathrm{C}-\mathrm{N}$ and N-C-N bonding character within the ligands (Figure 21e). HOMO-7 is weakly bonding between metal and ligands $02,012,017$ and 022 , and antibonding with respect to 07 and 027 . The internal bonding nature of the ligands is unclear for HOMO-7 as some ligands appear antibonding between $\mathrm{p}$ orbitals on the $\mathrm{N}$ 's and the $\mathrm{O}$, while some are bonding due to favourable orientation of the p-orbitals (Figure 21d). HOMO-6 is weakly bonding between the metal and ligands 07, 012, 017 and 027, and antibonding to $\mathrm{O} 2$ and $\mathrm{O} 22$. The ligands have a similar internal bonding nature as in $\mathrm{HOMO}-$ 7 (Figure 21c). The HOMO is antibonding between the metal and all ligands, and mostly antibonding between the nitrogens and oxygens within the ligands (Figure 21b). HOMO+1 is antibonding between metal and all ligands, but more weakly for 012 and 017 . There is $\mathrm{C}=\mathrm{O}$ bonding on some ligands and C-N antibonding on some ligands (Figure 21a). 

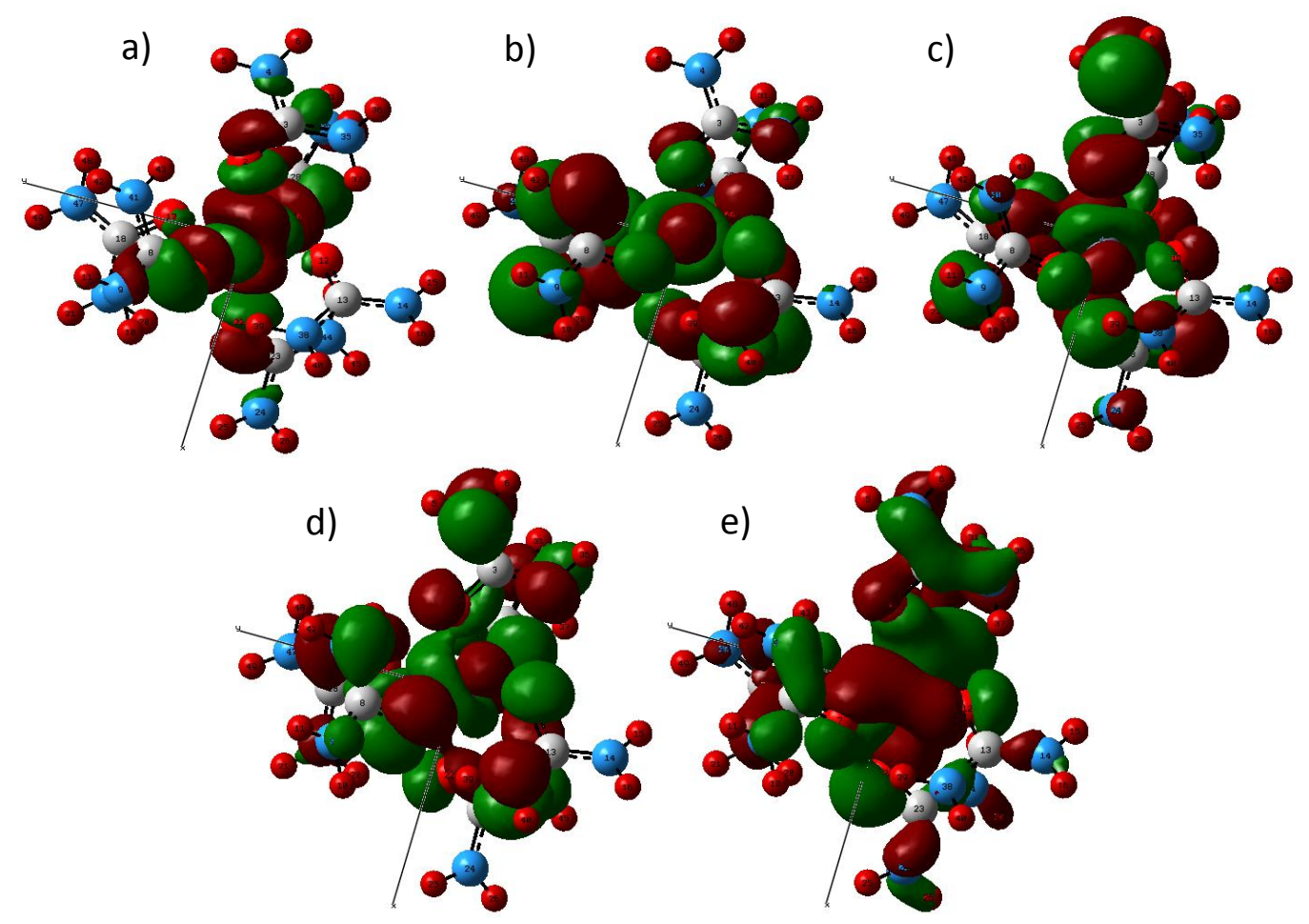

Figure 21: Relevant ground state MOs for $\left[\mathrm{Cr}\left(\mathrm{CONH}_{2}\right)_{2}\right]^{3+}$ : a) LUMO b) HOMO c) HOMO-6 d) HOMO-7 e) HOMO-20

The optimised geometry of the quartet excited state was calculated and found to also have no symmetry. The $\mathrm{Cr}-\mathrm{O}$ bond distance varies from $1.92 \AA$ to $2.47 \AA$, and the $\mathrm{C}=\mathrm{O}$ bonds vary from $1.25 \AA$ to $1.30 \AA$. The $\mathrm{C}-\mathrm{N}$ conjugated bonds vary from $1.32 \AA$ to $1.38 \AA$. The $\mathrm{Cr}-\mathrm{O}-\mathrm{C}$ bond angle is between $135^{\circ}$ and $150^{\circ}$. This means that in general the molecule looks the same as in the GS, but the bond angles and lengths are more widely spread, the longer bonds are slightly longer and the shorter ones are slightly shorter (Figure 22). There is a small increase of 0.04 in Mulliken charge on the metal.

a)

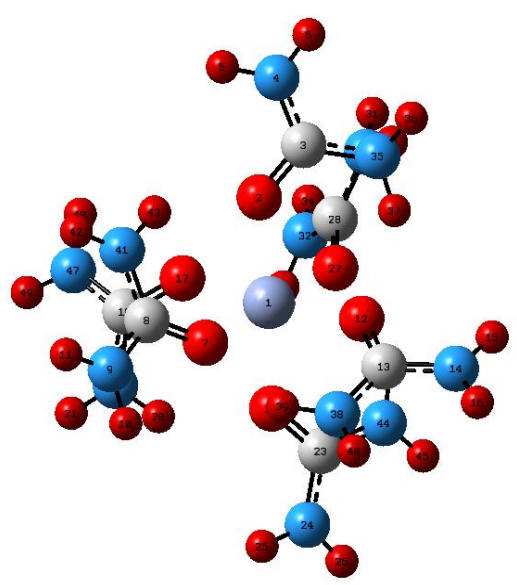

b)

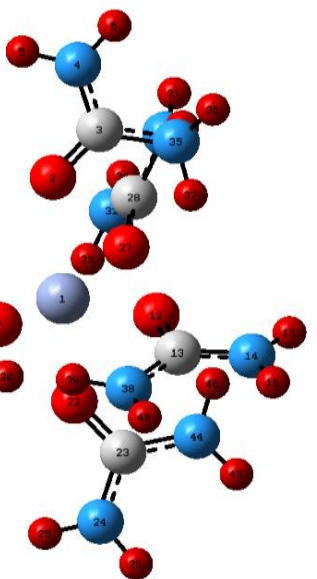

Figure 22: Optimised geometries of quartet hexaureachromate: a) GS b) ES 
The vertical excitation energy for quartet hexaureachromate was calculated to be $198 \mathrm{kJmol}^{-1}$. This is lowered by $27 \mathrm{kJmol}^{-1}$ due to vibrational relaxation of the excited state geometry, so the energy difference between the GS and ES is $171 \mathrm{kJmol}^{-1}$.

The vertical excitation energy for doublet hexaureachromate was calculated to be $35 \mathrm{kJmol}^{-1}$, however the optimised doublet excited state could not be obtained due to high computational cost. 


\section{Chromium Acetylacetonate [Cr(acac) 3 ]}

The quartet ground state of chromium acetylacetonate was calculated to have $C_{3}$ symmetry (Figure 23). The $\mathrm{Cr}=\mathrm{O}$ bond distances are $1.97 \AA$, the $\mathrm{C}=\mathrm{O}$ are formal double bonds at $1.27 \AA$. The central three $\mathrm{C}$ in each ligand are conjugated together with a C-C bond at $1.40 \AA$ and the terminal C-C bonds are all $1.51 \AA$ A. The Mulliken charges are $\mathrm{Cr}=1.56, \mathrm{O}=-0.52$, carbonyl carbons are 0.38 , central carbon is -0.47 and methyl carbons are -0.67 .

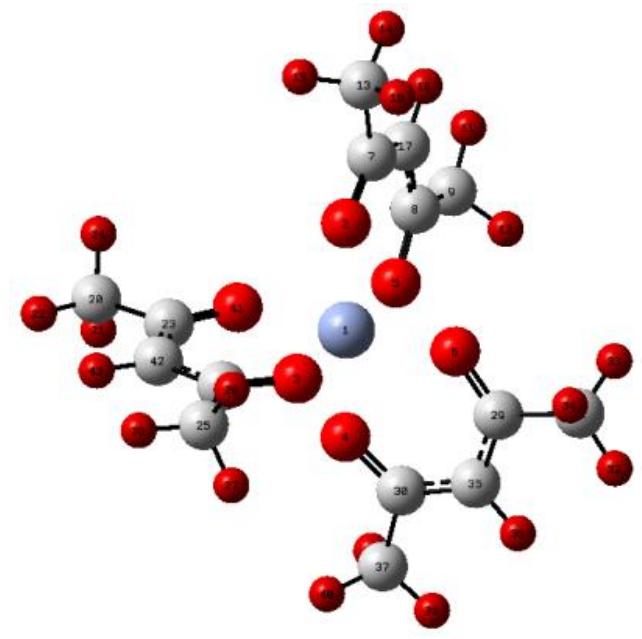

Figure 23: Chromium acetylacetonate

The doublet lowest lying state has no symmetry. $\mathrm{The} \mathrm{Cr}=\mathrm{O}$ bond distances are $1.97 \AA$ for $\mathrm{O} 2$ and $04,1.96 \AA$ for 05 and 06 , and $1.97 \AA$ for 03 and 041 . The $\mathrm{C}=\mathrm{O}$ are formal double bonds at $1.27 \AA$. The central three $C$ in each ligand are conjugated together with the $C-C$ bonds at $1.40 \AA$ and the terminal $\mathrm{C}-\mathrm{C}$ bonds are all $1.51 \AA$. The Mulliken charges are $\mathrm{Cr}=1.54$, $\mathrm{O}=-0.52$, carbonyl $\mathrm{C}=0.38$, central carbon $=-0.47$ and methyl carbons $=-0.67$.

The predicted UV-Vis spectrum for quartet chromium acetylacetonate shows two peaks at $275 \mathrm{~nm}$ with $\varepsilon$ of 9000 and at $229 \mathrm{~nm}$ with $\varepsilon$ of 9800 . There are also a number of weaker transitions which are hidden by the main peaks such as the one at $320 \mathrm{~nm}$ with $\varepsilon$ of 2100 (Spectrum 7a). The transition corresponding to the first excited state appears at $493 \mathrm{~nm}$ but has a very small $\varepsilon$. The doublet UV/Vis spectrum shows 2 main peaks at $304 \mathrm{~nm}$ with $\varepsilon$ of 6300 and at $253 \mathrm{~nm}$ with $\varepsilon$ of 4000 . There is also a few notable transitions on the lower energy side of the peak such as the one at $343 \mathrm{~nm}$ with an $\varepsilon$ of 5000, and a small low energy transition at $423 \mathrm{~nm}$ which has an $\varepsilon$ of 800 (Spectrum $7 \mathrm{~b}$ ). The doublet state peaks are comprised of many more allowed transitions than the quartet peaks, where each is mostly made up of a just few major transitions. The doublet first excited state appears at $3844 \mathrm{~nm}$ but has a very small $\varepsilon$. 


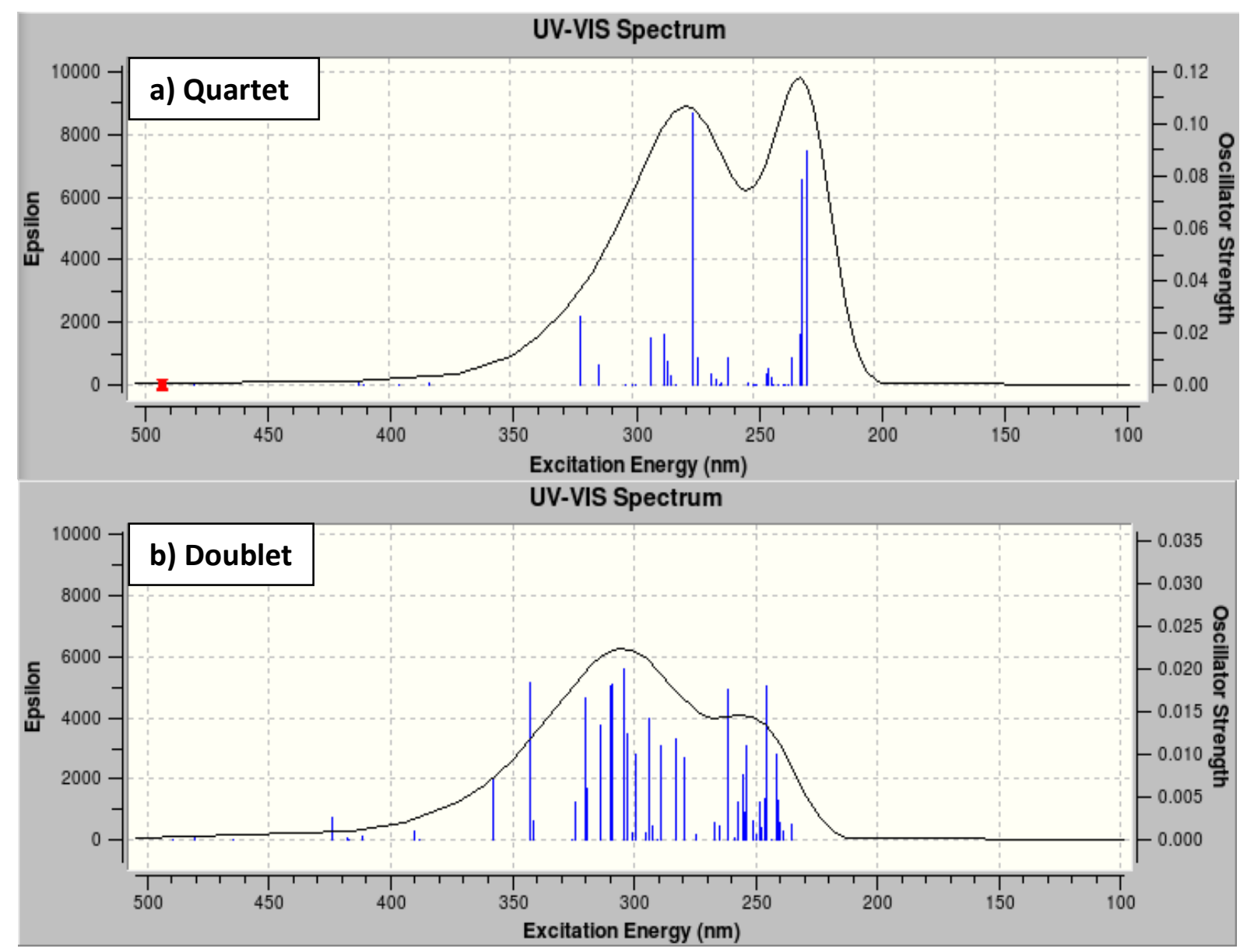

Spectrum 7: UV-Vis spectrum of quartet chromium acetylacetonate

The experimental data for quartet chromium acetylacetonate shows peaks at $253 \mathrm{~nm}$, $274 \mathrm{~nm}, 296 \mathrm{~nm}, 340 \mathrm{~nm}, 380 \mathrm{~nm}$, and $543 \mathrm{~nm}$ with low extinction coefficients [32]. The predicted peaks are at $229 \mathrm{~nm}, 275 \mathrm{~nm}$, and there are also weaker transitions at $320 \mathrm{~nm}$ and at about $490 \mathrm{~nm}$. When adjusted for the $0.22 \mathrm{eV}$ error, these peaks shift to $264 \mathrm{~nm}$, $289 \mathrm{~nm}, 340 \mathrm{~nm}, 537 \mathrm{~nm}$. Comparing these adjusted values to the experimental data we get a remarkably good fit. Remodelling in aqueous solution caused negligible change to the position of the transitions.

The quartet first excited state configuration arises from 12 excited configurations, however for simplicity only the 4 main configurations have been considered as they contribute $80 \%$ of the first excited state configuration between them. There are two from HOMO-4(A) $\rightarrow$ LUMO+2(E) (38\%), and two from HOMO(E) $\rightarrow$ LUMO+2(E) (42\%). Visual determination of the nature of the MOs shows that one of the degenerate MOs in the HOMO-4 is bonding between the metal and all ligands and reinforces the conjugation in the 
centre of the ligand. There is also a bonding mode which extends from the metal, through one of the oxygens on each ligand, across the carbonyl carbon and onto the terminal carbon on one side of the ligand only for $\mathrm{O} 3$ and $\mathrm{O} 4$ ligands, but extends across both sides of the ligand for the 02/O5 ligand (Figure 24c). The other $\mathrm{MO}$ in HOMO-4 shows the same bonding, but extending across the opposite side of each ligand as was observed in the other MO. Ligand $02 / 05$ still shows equal and $C_{2}$ symmetric bonding modes on both sides of the ligand (Figure 24c). One of the MOs in the HOMO is weakly bonding between the metal and ligand $02 / 05$, but is antibonding to the other two ligands. The ligands show strong bonding between the central conjugated carbons and a strong $\mathrm{C}=\mathrm{O}$ antibonding mode, but there is little density on ligand 02/O5 (Figure 24b). The other MO in the HOMO shows the same bonding patterns, but this time is weakly bonding to 03/041 and 04/06 and is antibonding to $02 / 05$. The ligands still show strong conjugated bonding in the central carbons and a strong $\mathrm{C}=\mathrm{O}$ antibonding mode (Figure $24 \mathrm{~b}$ ). One of the $\mathrm{MOs}$ in the $\mathrm{LUMO}+2$ is antibonding between the metal and ligating atoms $\mathrm{O} 2, \mathrm{O} 3, \mathrm{O} 4$ and $\mathrm{O} 5$ and is non-interacting with $\mathrm{O} 6$ and 041. There is some $\mathrm{C}=\mathrm{O}$ bonding in $\mathrm{O} 2, \mathrm{O} 4, \mathrm{O} 6$ and $\mathrm{O} 41$ (Figure 24a). The other $\mathrm{MO}$ in the LUMO+2 is antibonding between the metal and all ligating atoms, but more strongly towards $\mathrm{O} 4$ and $\mathrm{O} 6$ (Figure 24a).

As the LUMO+2 is antibonding between the metal and the ligands we expect to see all the $\mathrm{Cr}-\mathrm{O}$ bond distances increase. There will also be a charge density shift onto the metal as the bulk of the electron accepting molecular orbitals is on the metal, whereas the donating MO's have a lot of density on the ligands. This may also affect the strength of the C-C bonds in the ligands as the conjugated carbon to carbon bonding modes aren't present in the LUMO+2.

The vertical excitation energy for quartet chromium acetylacetonate was found to be $243 \mathrm{kJmol}^{-1}$ and the vertical excitation energy for doublet chromium acetylacetonate was found to be $31 \mathrm{kJmol}^{-1}$. The optimised excited states were unable to be calculated due to time constraints and computational expense. 

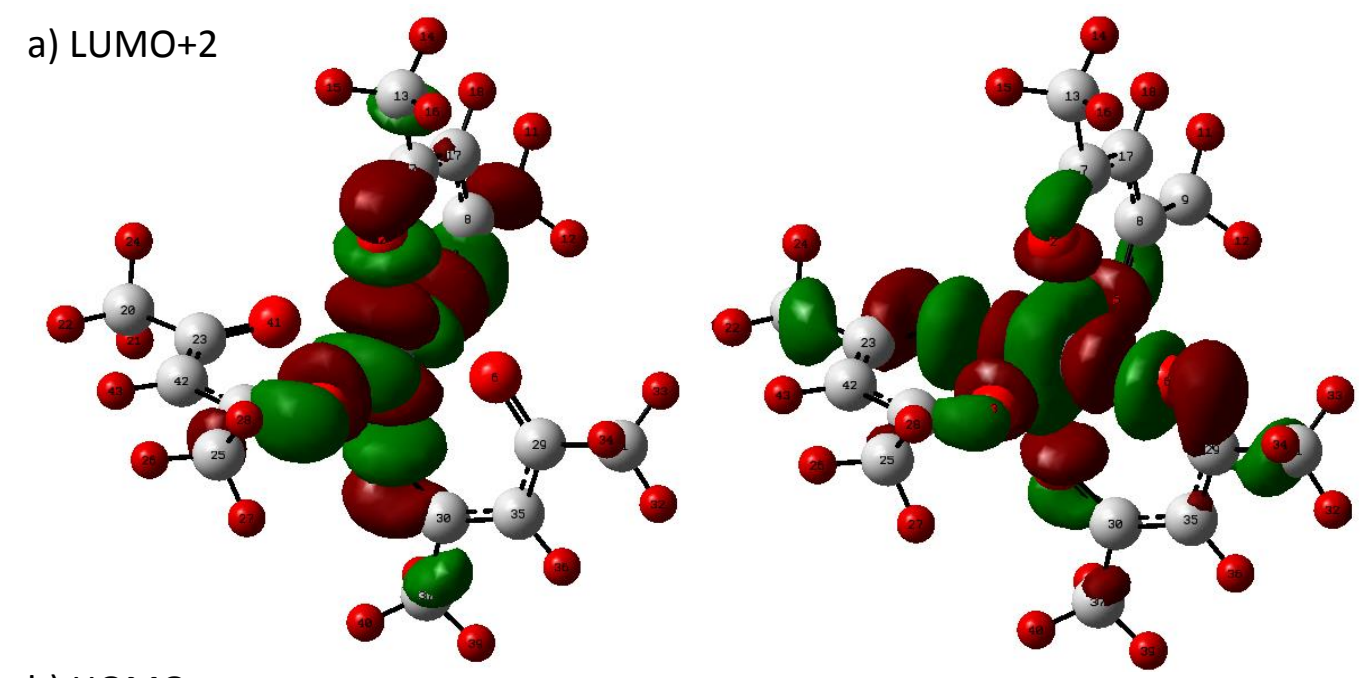

b) $\mathrm{HOMO}$
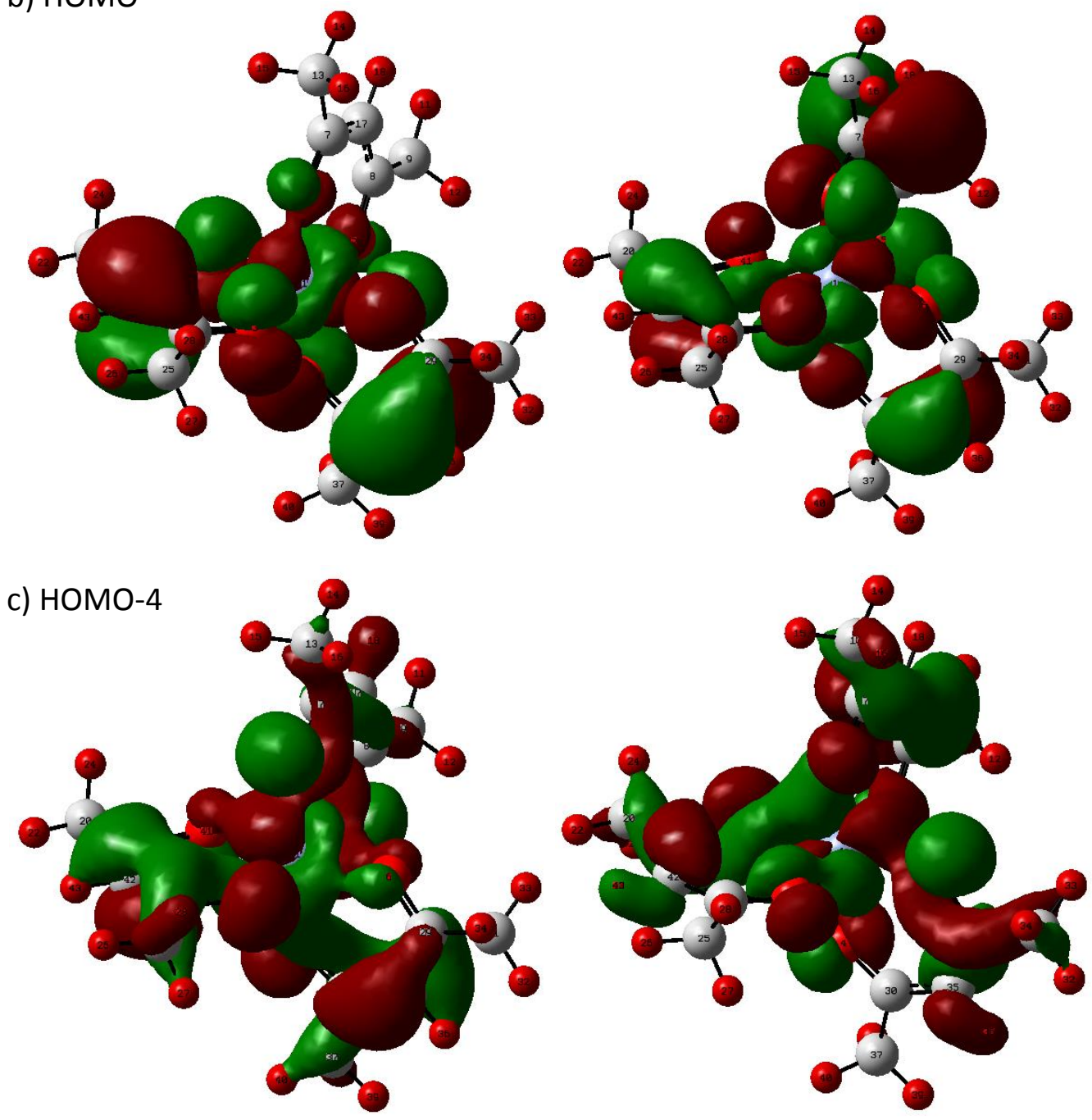

Figure 24: Relevant ground state MOs for $\left[\mathrm{Cr}(\mathrm{acac})_{3}\right]$ : a) LUMO+2 b) HOMO c) HOMO-4 


\section{Hexacyanochromate $\left[\mathrm{Cr}(\mathrm{CN})_{6}\right]^{3-}$}

The quartet ground state of hexacyanochromate has $\mathrm{O}_{\mathrm{h}}$ symmetry (Figure 25). The $\mathrm{Cr}$-C bond distance is $2.14 \AA$ and the $\mathrm{C}-\mathrm{N}$ is triply bonded at a distance of $1.17 \AA$. The Mulliken charges are $\mathrm{Cr}=1.05, \mathrm{C}=-0.22$ and $\mathrm{N}=-0.45$.

The doublet lowest lying state has $D_{4 h}$ symmetry. The $\mathrm{Cr}-\mathrm{C}$ bond distances are $2.12 \AA$ on the yz plane and 2.13 $\AA$ along the $\mathrm{x}$ axis. The $\mathrm{C}-\mathrm{N}$ bond distance is $1.17 \AA$ for all ligands. The $\mathrm{C}-\mathrm{Cr}-\mathrm{C}$ angles are all $90^{\circ}$ so only the

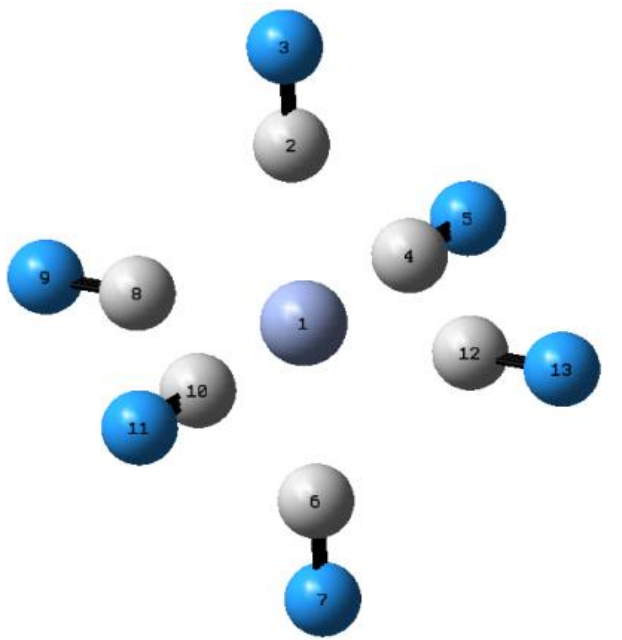

Figure 25: Hexacyanochromate slight $\mathrm{x}$-axis extension prevents this molecule from being octahedral. The Mulliken charges are $\mathrm{Cr}=1.02, \mathrm{C}=-0.22$ and $\mathrm{N}=-0.45$.

The predicted UV/Vis spectrum for quartet hexacyanochromate has two main peaks at 243 $\mathrm{nm}$ with $\varepsilon$ of 4100 and at $202 \mathrm{~nm}$ with $\varepsilon$ of 6300 . There is also a smaller peak between these two at $220 \mathrm{~nm}$ with $\varepsilon$ of 3500 (Spectrum 8a). The first excited state appears at $382 \mathrm{~nm}$ but is symmetry forbidden. The doublet UV/Vis spectrum is quite similar, with two main peaks at $267 \mathrm{~nm}$ with $\varepsilon$ of 3000 and at $233 \mathrm{~nm}$ with $\varepsilon$ of 3500 . There is also a notable allowed transition between the two peaks which is hidden by the overlap which is at $250 \mathrm{~nm}$ with epsilon 1500, and a shoulder at $297 \mathrm{~nm}$ with $\varepsilon$ of 1500 (Spectrum 8b). The doublet first excited state appears at $3706 \mathrm{~nm}$ but is symmetry forbidden.

The quartet first excited state configuration arises from two main excited configurations which between them contribute $91 \%$ of the excited state; both configurations arise from HOMO( $\left.T_{2 g}\right) \rightarrow$ LUMO(Eg) (91\%). Visual determination of the nature of the MOs shows that the $\mathrm{HOMO}$ is antibonding between the metal and the ligands on the xz plane, and non-bonding with respect to the $\mathrm{y}$-axis ligands. There is also a strong $\mathrm{C}-\mathrm{N}$ bonding pi interaction in the $\mathrm{xy}$ plane ligands (Figure 26b). One of the MOs in the LUMO is sigma antibonding between the metal and all ligands, but more strongly on the yz plane. There is also a $\mathrm{C}-\mathrm{N}$ bonding sigma interaction in all ligands. LUMO+1 is the same as the LUMO but oriented along the xy plane instead of the yz plane (Figure 26a). 


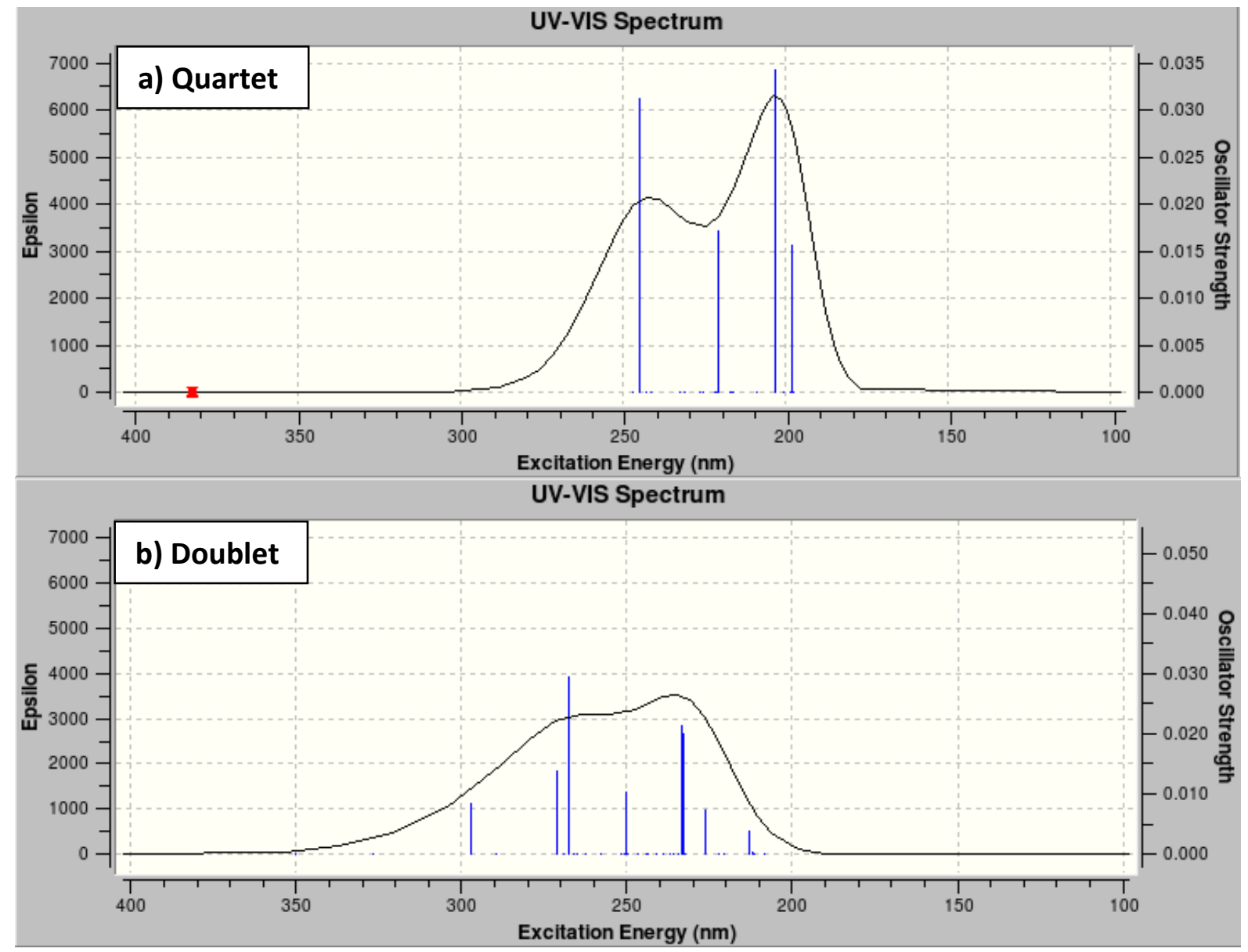

Spectrum 8: UV/Vis spectrum of hexacyanochromate

The experimental data for quartet hexacyanochromate in aqueous solution shows peaks at $308 \mathrm{~nm}$ and $377 \mathrm{~nm}$ [48]. The predicted spectrum has the peaks at $202 \mathrm{~nm}$ and $243 \mathrm{~nm}$. When adjusted for the $0.22 \mathrm{eV}$ error these shift to $210 \mathrm{~nm}$ and $254 \mathrm{~nm}$ which is not great agreement with experiment being about $100 \mathrm{~nm}$ off. Remodelling in aqueous solution caused negligible change to the position of the transitions. 
a) LUMO

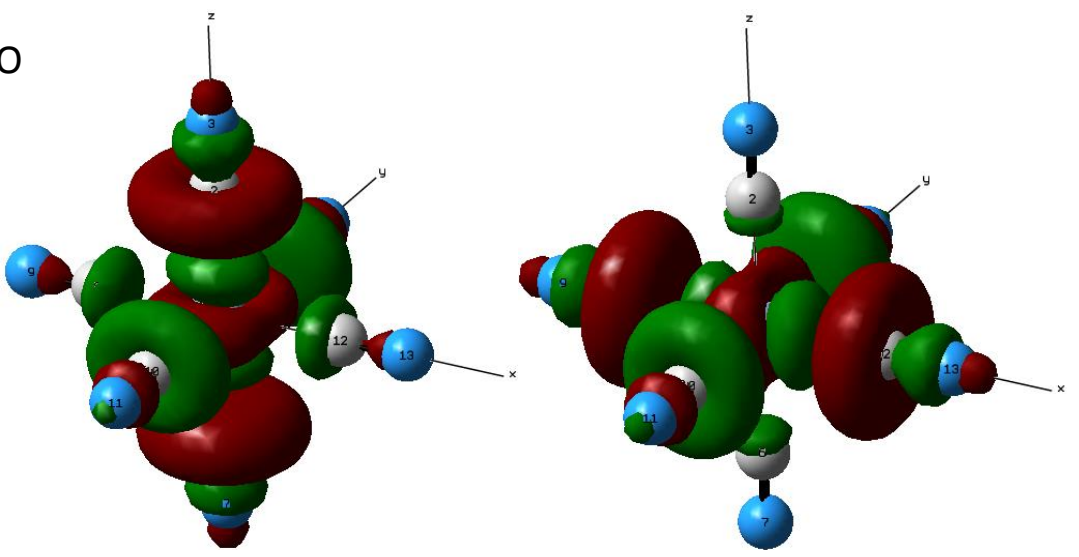

b) HOMO

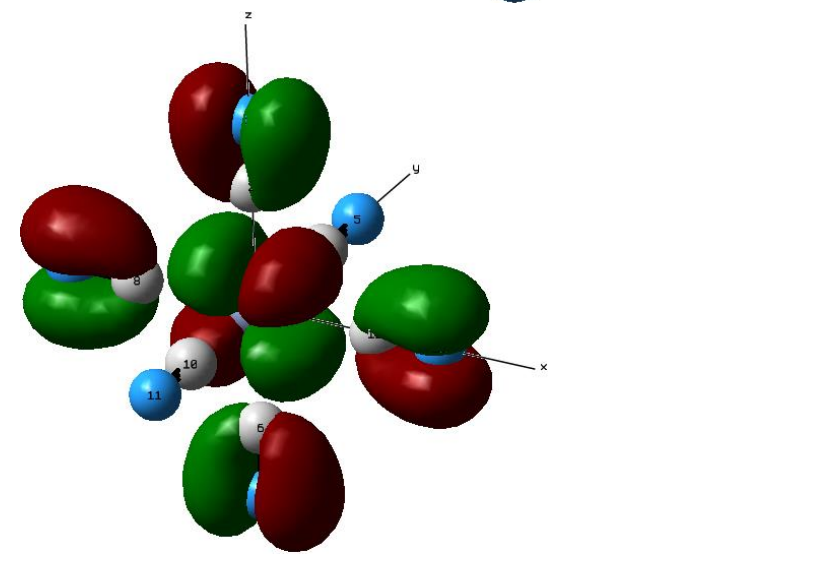

Figure 26: Relevant ground state MOs for $\left[\mathrm{Cr}(\mathrm{CN})_{6}\right]^{3-}$ quartet: a) LUMO b) HOMO

One of the HOMO $\rightarrow$ LUMO configurations contributes about 3 times as much towards the excited state configuration as the other and so has a much larger effect on the excited state structure. In general this transition should cause an elongation of the $\mathrm{y}$-axis $\mathrm{Cr}-\mathrm{C}$ bond and a contraction of the $\mathrm{y}$-axis $\mathrm{C}-\mathrm{N}$ triple bond. The reverse should be seen along the z-axis with a decrease in $\mathrm{Cr}-\mathrm{C}$ antibonding character and a decrease in $\mathrm{C}-\mathrm{N}$ bonding character. The $\mathrm{x}$-axis will only see a small effect from the transition as the only difference is that the $\mathrm{Cr}-\mathrm{C}$ antibonding, and the $\mathrm{C}-\mathrm{N}$ bonding is sigma instead of pi, so will vary only by the size of the interaction, which appears comparable. The other HOMO $\rightarrow$ LUMO configuration is less contributing and so will have a smaller effect on the structure of the excited state, but in general this configuration should cause an elongation of the $\mathrm{y}$-axis $\mathrm{Cr}-\mathrm{C}$ bond and a contraction of the $\mathrm{y}$-axis $\mathrm{C}-\mathrm{N}$ triple bond. The reverse should be seen along the $\mathrm{x}$-axis with a decrease in $\mathrm{Cr}-\mathrm{C}$ antibonding character and a decrease in $\mathrm{C}-\mathrm{N}$ bonding character. The z-axis will only see a small effect as the only difference is that the $\mathrm{Cr}-\mathrm{C}$ antibonding, and the $\mathrm{C}-\mathrm{N}$ bonding is sigma instead of pi, so will vary only by the size of the interaction, which appears comparable. This means that both configurations will have similar effects on the $y$-axis ligands, while the other two axes have a proportional reverse effect. Z-axis ligands should 
see a larger effect than $\mathrm{x}$-axis ligands because the changes to $\mathrm{z}$-axis ligands are due to the HOMO-1 $\rightarrow$ LUMO+1 transition. Overall, the $y$-axis ligands should move much further away from the metal and have a shorter $\mathrm{C}-\mathrm{N}$ bond, the $\mathrm{x}$-axis ligands should move slightly closer to the metal with a slightly longer $\mathrm{C}-\mathrm{N}$ bond, and z-axis ligands should move closer to the metal and have a longer $\mathrm{C}-\mathrm{N}$ bond. This will give the excited state $D_{2 h}$ symmetry. The charge density should not be affected greatly as there is no major shift of $\mathrm{MO}$ density between the metal and the ligands.

The optimised geometry of the quartet first excited state was calculated and it was found to have $D_{2 h}$ symmetry as predicted. The bond lengths are $\mathrm{Cr}-\mathrm{C} 8 / \mathrm{C} 12=2.45 \AA, \mathrm{Cr}-\mathrm{C} 2 / \mathrm{C} 5=2.19 \AA$ and $\mathrm{Cr}-\mathrm{C} 4 / \mathrm{C} 10=2.12 \AA$ and $\mathrm{C}-\mathrm{N}$ are $1.17 \AA$, $1.16 \AA$ and $1.17 \AA$ for $\mathrm{x}, \mathrm{y}$ and $\mathrm{z}$ axes respectively. The Mulliken charges are $\mathrm{Cr}=1.27, \mathrm{C}_{\mathrm{x} \text {-axis }}=-0.34, \mathrm{C}_{\mathrm{y} \text {-axis }}=-0.26, \mathrm{C}_{\mathrm{z} \text {-axis }}=-0.22, \mathrm{~N}_{\mathrm{x} \text {-axis }}=-0.46$, $\mathrm{N}_{\mathrm{y} \text {-axis }}=-0.43$ and $\mathrm{N}_{\mathrm{z} \text {-axis }}=-0.43$. This is just as was predicted in the orbital analysis above. As the ground state was $\mathrm{O}_{h}$, the axis assignments are arbitrary, and so in general we see one axis lengthen considerably, one lengthen slightly and one shorten slightly.

The vertical excitation energy for quartet hexacyanochromate was calculated to be $313 \mathrm{kJmol}^{-1}$. The energy was lowered by $50 \mathrm{~kJ} \mathrm{~mol}^{-1}$ due to vibrational relaxation, so the energy difference between the GS and ES is $263 \mathrm{kJmol}^{-1}$.

The vertical excitation energy for doublet hexacyanochromate was calculated to be $32 \mathrm{kJmol}^{-1}$. The doublet optimised excited state was calculated but the data was contradictory, stating that the vibrational relaxation lowered the energy by $60 \mathrm{kJmol}^{-1}$ which is almost double the $E_{\text {vert }}$. It is possible that the initial calculation converged to the excited state and that the TD-DFT calculation has found the true lowest lying state, except that the calculated excitation energy from lowest lying to excited triplet state is a positive number. This suggests that the vertical excitation excites to a higher energy state which then relaxes down to a lower energy minimum than the lowest lying state which is unphysical. 


\section{Chromium Hexaimidazolidone $\left[\operatorname{Cr}\left(\mathrm{C}_{3} \mathrm{H}_{5} \mathrm{~N}_{2} \mathrm{O}\right)_{6}\right]^{3+}$}

The quartet ground state of chromium hexaimidazolidone was calculated to have $C_{3}$ symmetry (Figure 27). The $\mathrm{Cr}-\mathrm{O}$ bond distances are all $2.00 \AA$ and the carbonyl lengths are $1.29 \AA$ A. The OC-N bond distances are $1.35 \AA$. The $\mathrm{C}=\mathrm{C}$ bond at the back of each ligand is also $1.35 \AA$. The $\mathrm{O}-\mathrm{Cr}-\mathrm{O}$ angles are within $5^{\circ}$ of $90^{\circ}$, and the $\mathrm{Cr}-\mathrm{O}-\mathrm{C}$ angle is $131^{\circ}$. The Mulliken charges are $\mathrm{Cr}=1.65, \mathrm{O}=-0.64$, carbonyl $\mathrm{C}=0.70, \mathrm{~N}=-0.55$ or -0.56 and other $\mathrm{C}$ in each ligand are 0.05 and -0.07 . The $\mathrm{H}$ bonded to the nitrogens have a

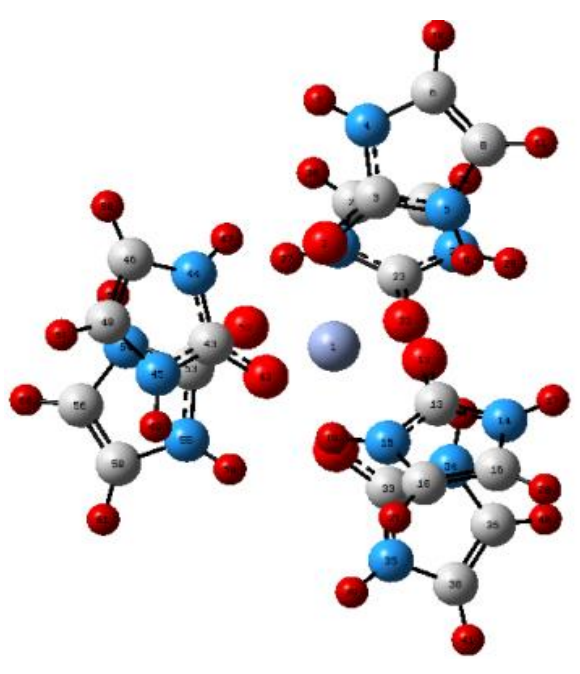

Figure 27: Chromium hexaimidazolidone charge of 0.40 and the $\mathrm{C}=\mathrm{C}$ hydrogens have a charge of 0.29 and 0.30 .

The doublet lowest lying state also has $C_{3}$ symmetry. The $\mathrm{Cr}$-O bond distances are all $2.00 \AA$ and the carbonyl lengths are $1.28 \AA$. The OC-N bond distances $1.35 \AA$. The $\mathrm{C}=\mathrm{C}$ bond at the back of each ligand is also $1.35 \AA$. The $\mathrm{O}-\mathrm{Cr}-\mathrm{O}$ angles are within $5^{\circ}$ of $90^{\circ}$, and the $\mathrm{Cr}-\mathrm{O}-\mathrm{C}$ angle is $131^{\circ}$. The Mulliken charges are $\mathrm{Cr}=1.62, \mathrm{O}=-0.64$, carbonyl $\mathrm{C}=0.70, \mathrm{~N}=-0.55$ or -0.56 and other $C$ in each ligand are -0.05 and -0.07 . The $H$ bonded to nitrogen have a charge of 0.40 and the $\mathrm{C}=\mathrm{C}$ hydrogens have a charge of 0.29 and 0.30 . This means that the lowest lying doublet state is almost identical to the quartet GS.

The predicted UV-Vis spectrum for quartet chromium hexaimidazolidone has 2 main peaks at $336 \mathrm{~nm}$ with $\varepsilon$ of 10000 and at $250 \mathrm{~nm}$ with $\varepsilon$ of 2200 . There is also a smaller transition at $381 \mathrm{~nm}$ with $\varepsilon$ of 2000 which is hidden in the main peak (Spectrum 9a). The quartet first excited state appears at $620 \mathrm{~nm}$ but is symmetry forbidden. The doublet UV-Vis spectrum has one main peak. The main peak is at $392 \mathrm{~nm}$ with $\varepsilon$ of 8000 and there are two notable transitions at 449 and $458 \mathrm{~nm}$ with $\varepsilon$ of 4800 which form a shoulder on the peak. There is also a tail at the higher energy side of the $392 \mathrm{~nm}$ peak made up of transitions with $\varepsilon$ values below 1000 (Spectrum 9b). This means that chromium hexaimidazolidone is one of the few molecules investigated which clearly absorbs into the visible region. The doublet first excited state appears at $3512 \mathrm{~nm}$ but is symmetry forbidden. 


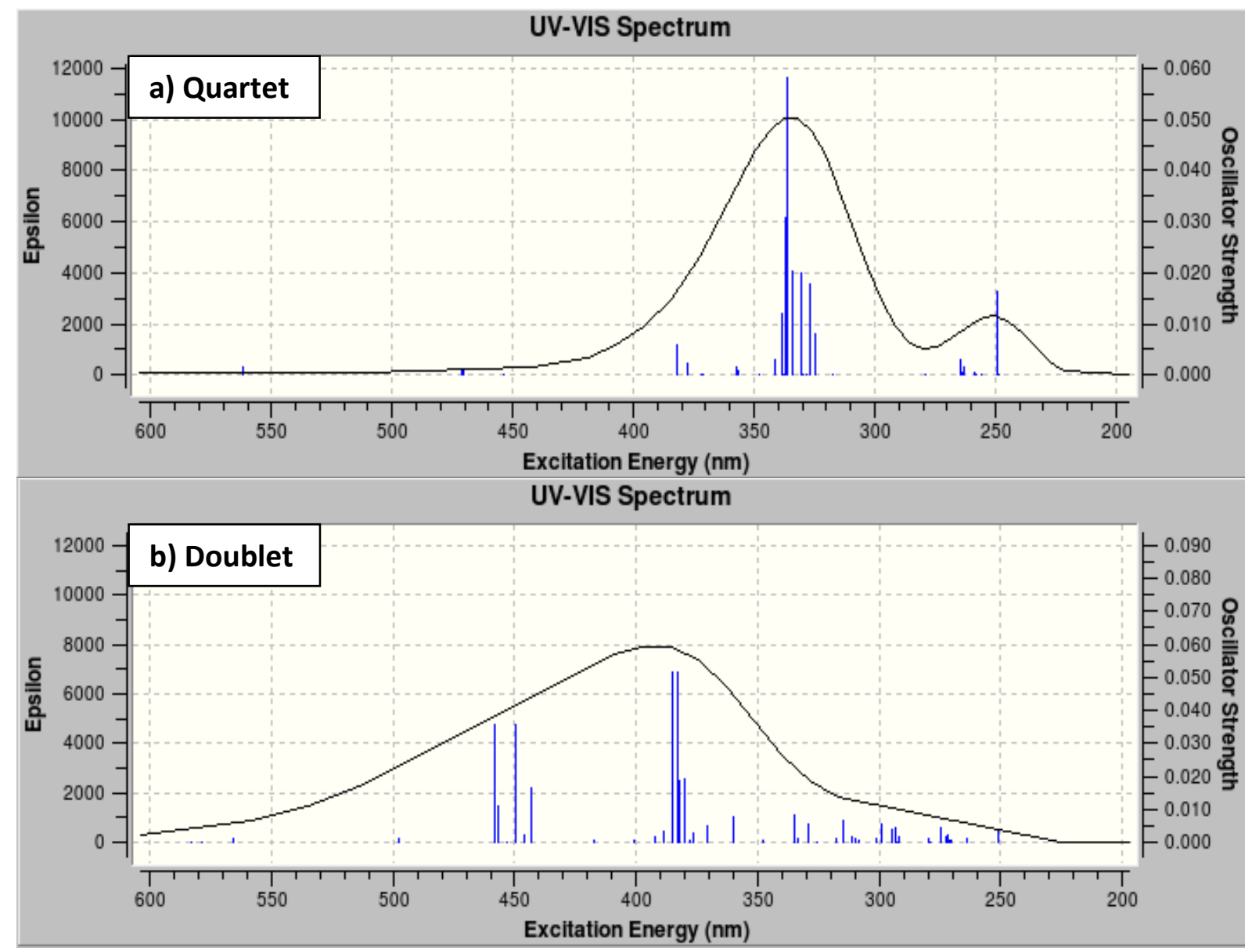

Spectrum 9: UV-Vis spectrum of chromium hexaimidazolidone

The experimental data for quartet chromium hexaimidazolidone shows peaks at $443 \mathrm{~nm}$ and $620 \mathrm{~nm}$ [49]. The predicted peaks are at $250 \mathrm{~nm}$ and $336 \mathrm{~nm}$, however the experimental data shows very small extinction coefficients so the theoretical peaks of interest may in fact be the ones which are barely visible or hidden under the main peak on Spectrum 9. These appear at $382 \mathrm{~nm}, 470 \mathrm{~nm}$ and $561 \mathrm{~nm}$ which when adjusted for the $0.22 \mathrm{eV}$ error are shifted to $409 \mathrm{~nm}, 512 \mathrm{~nm}$ and $623 \mathrm{~nm}$. Remodelling in aqueous solution caused a notable change to the positions of these peaks, shifting them to $357 \mathrm{~nm}, 461 \mathrm{~nm}$ and $556 \mathrm{~nm}$. These new values adjust to $381 \mathrm{~nm}, 502 \mathrm{~nm}$ and $617 \mathrm{~nm}$. This is interesting as the lowest energy peaks are in good agreement, but the higher energy peaks are not as close.

The quartet first excited state configuration arises from 11 electronic configurations, most of which contribute less than $5 \%$ to the excited state. There are, however, 4 main configurations which together contribute $73 \%$ of the excited state. These are HOMO$5 \rightarrow$ LUMO+1 (31\%), two from HOMO-4 $\rightarrow$ LUMO (12\%) and HOMO $\rightarrow$ LUMO (30\%). Visual 
determination of the nature of the MOs shows that HOMO-5 is antibonding between the metal $d_{z^{2}}$ orbital and all ligands. There is a strong pi bonding mode on the carbonyl bond and also some conjugation visible across the $\mathrm{C}=\mathrm{C}$ and one of the nitrogens in each ligand (Figure 28d). One of the MOs in HOMO-4 shown the metal is antibonding with respect to 022 and $\mathrm{O} 42$ but bonding with respect to the other four ligands. The other $\mathrm{MO}$ in the HOMO-4 shows the metal is antibonding with respect to $\mathrm{O} 2$ but bonding with respect to the other ligands. It also has some carbonyl bonding character (Figure 28c). HOMO has a small $\mathrm{d}_{\mathrm{z}^{2}}$ orbital on the metal which is antibonding to all ligands. The bulk of this MO lies on the ligands, where there is a $\mathrm{C}=\mathrm{O}$ antibonding interaction, an $\mathrm{N}-\mathrm{C}-\mathrm{N}$ bonding interaction, a $\mathrm{C}=\mathrm{C}$ bonding interaction and an $\mathrm{N}-\mathrm{C}=\mathrm{C}$ antibonding interaction (Figure 28b). One of the $\mathrm{MOs}$ in the LUMO shows an antibonding interaction between the metal and all ligands, except for $\mathrm{O} 22$ and $\mathrm{O} 42$ which are non-bonding. There is also a weak $\mathrm{C}=\mathrm{O}$ bonding interaction. The other MO in the LUMO is antibonding between the metal and all ligands, but more strongly with respect to $\mathrm{O} 22$ and 042 (Figure 28a).

The HOMO-6 $\rightarrow$ LUMO transition will have a destabilising effect on the ligands, as the intraligand bonding modes in HOMO-6 are not present in the LUMO. The metal to ligand interaction is antibonding for both configurations, except for $\mathrm{O} 22$ and $\mathrm{O} 42$ which are nonbonding in the LUMO. The HOMO-4 $\rightarrow$ LUMO transition will shift the ligands away from the metal as the metal-ligand interaction changes from bonding to antibonding. The HOMO $\rightarrow$ LUMO transition will definitely see a structural change in the ligands. The $\mathrm{C}=\mathrm{C}$ bonding mode is gone, as is the N-C-N bonding mode. Corollary to this, the carbonyl and N$\mathrm{C}=$ antibonding interactions are also gone, and there is a weak carbonyl bonding interaction instead. This means two things: there will be an overall shift of electron density from ligands to the metal and the oxygens, and the ligands will undergo internal shifts, with the $\mathrm{C}=\mathrm{O}$ and $\mathrm{N}-\mathrm{C}=$ bonds shortening and the $\mathrm{C}=\mathrm{C}$ and $\mathrm{N}-\mathrm{C}-\mathrm{N}$ bonds getting longer. All 3 molecular orbitals are antibonding between the metal and the ligands, so there should only be minor changes to the $\mathrm{Cr}-\mathrm{O}$ distances. As the LUMO has the bulk of the electron density on the metal and the coordinating oxygens, we should see an increase in negative charge at these positions. This suggests that this is a ligand to metal charge transfer transition. 
a) LUMO
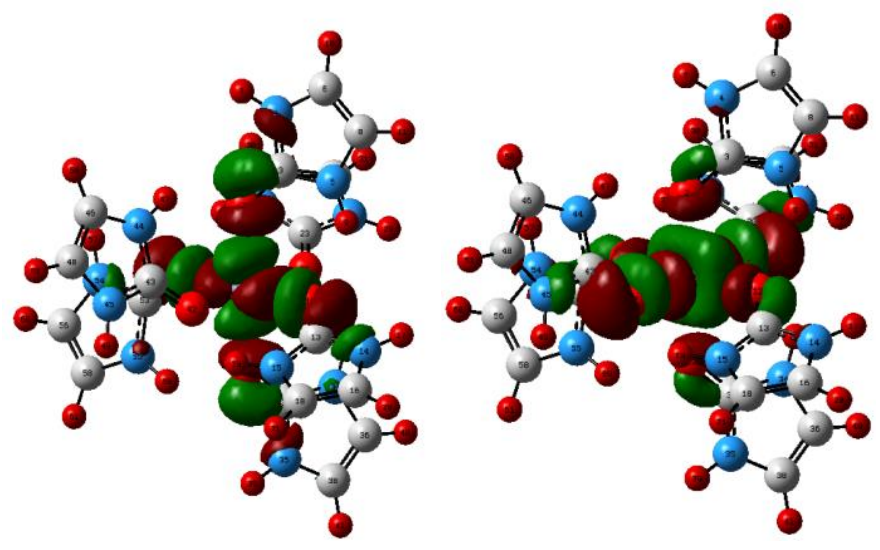

b) HOMO

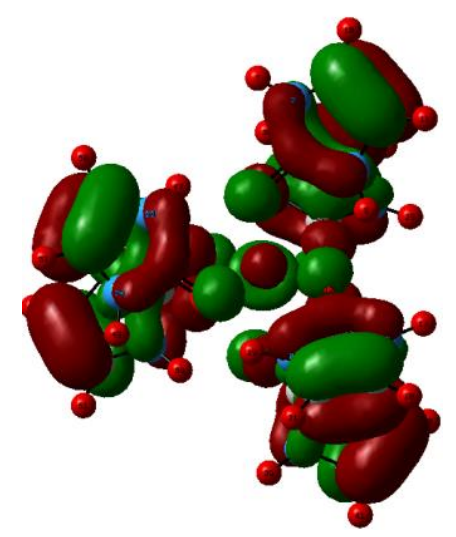

c) HOMO-5
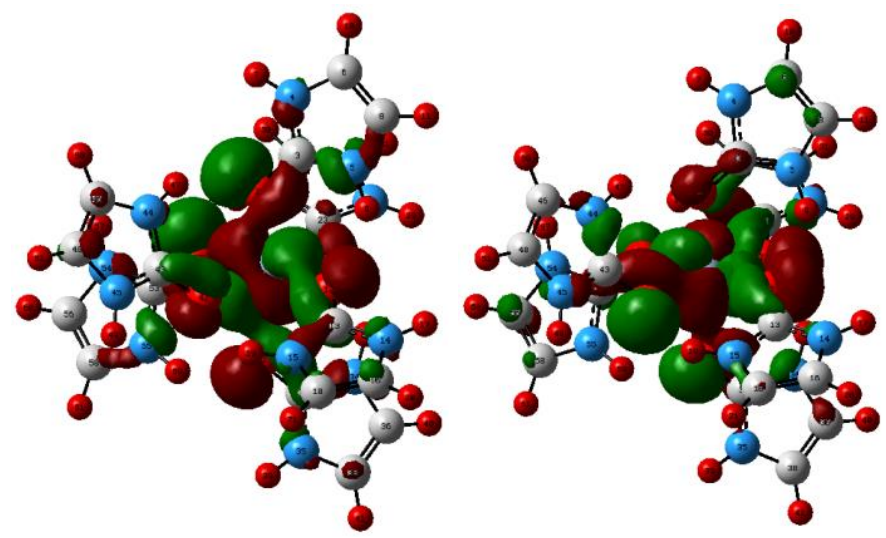

d) HOMO-6

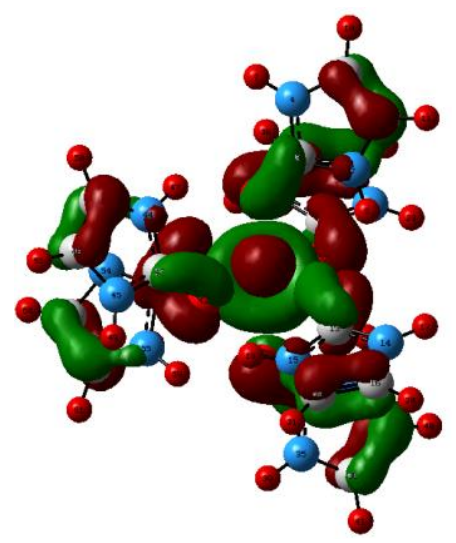

Figure 28: Relevant ground state MOs for $\left[\mathrm{Cr}\left(\mathrm{C}_{3} \mathrm{H}_{5} \mathrm{~N}_{2} \mathrm{O}\right)_{6}\right]^{3-}$ quartet: a) LUMO b) HOMO c) HOMO-5 d) HOMO-6 
The vertical excitation energy for quartet chromium hexaimidazolidone was calculated to be $193 \mathrm{kJmol}^{-1}$ and the vertical excitation energy for doublet chromium hexaimidazolidone was calculated to be $34 \mathrm{kJmol}^{-1}$. The optimised excited state of quartet and doublet chromium hexaimidazolidone were unable to be obtained in a reasonable time frame as the size and complexity of the molecule makes it very computationally expensive. 


\section{Trioxalatochromate $\left[\mathrm{Cr}\left(\mathrm{C}_{2} \mathrm{O}_{4}\right)_{3}\right]^{3-}$}

The quartet ground state of trioxalatochromate was found to have $D_{3}$ symmetry (Figure 29). The $\mathrm{Cr}$-O bond distance is $2.00 \AA$, the coordinated $\mathrm{O}-\mathrm{C}$ is $1.29 \AA$, the $\mathrm{C}-\mathrm{C}$ is $1.58 \AA$ and the non-coordinated $\mathrm{O}-\mathrm{C}$ is $1.23 \AA$. The Mulliken charges are $\mathrm{Cr}=1.50$, coordinating $\mathrm{O}=-0.55, \mathrm{C}=0.27$, other $\mathrm{O}=-0.47$.

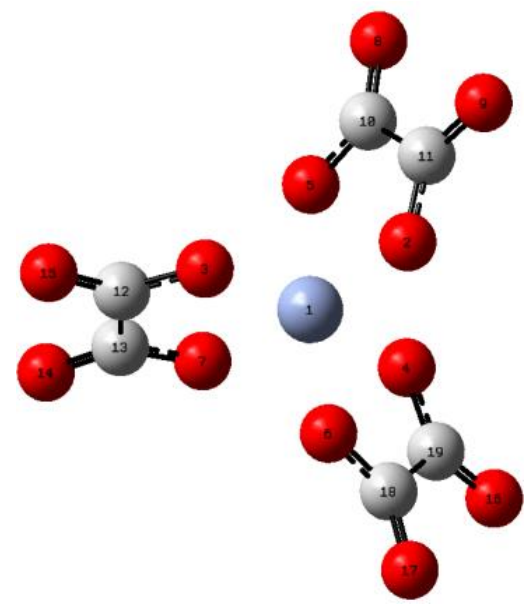

Figure 29: Trioxalatochromate symmetry. The $\mathrm{Cr}$-O bond distance is $2.00 \AA$, the coordinated $\mathrm{O}-\mathrm{C}$ is $1.29 \AA$, the $\mathrm{C}-\mathrm{C}$ is $1.58 \AA$ and the non-coordinated $\mathrm{O}-\mathrm{C}$ is $1.23 \AA$. The Mulliken atomic charges are $\mathrm{Cr}=1.48$, coordinating $\mathrm{O}=-0.55, \mathrm{C}=0.27$, other $\mathrm{O}=-0.47$. So the quartet and doublet lowest lying states are almost identical.

The predicted UV/Vis spectrum of quartet trioxalatochromate has one main peak at $226 \mathrm{~nm}$ with $\varepsilon$ of 8000 . There are also two notable transitions at around $270 \mathrm{~nm}$ which have $\varepsilon$ of 800 and form a tail on the left side of the peak. There is a very small transition at $409 \mathrm{~nm}$ with $\varepsilon$ of 250 which makes a small 'bump' in the visible region (Spectrum 9a). The quartet first excited state appears at $519 \mathrm{~nm}$ but is forbidden and so has a very small $\varepsilon$. The doublet UV/Vis spectrum has one main peaks which appears to actually be two peaks which overlap such that the lower energy peak forms a high shoulder on the higher energy peak. The lower energy peak is at $280 \mathrm{~nm}$ with $\varepsilon$ of 2000 and the higher energy peak is at $246 \mathrm{~nm}$ with $\varepsilon$ of 3200. There is also a pair of transitions at $434 \mathrm{~nm}$ which have $\varepsilon$ of 400 and form a bump in the visible region of the spectrum (Spectrum 9b). The doublet first excited state appears at $3766 \mathrm{~nm}$ but is forbidden.

The experimental data for quartet trioxalatochromate shows peaks at $417 \mathrm{~nm}$ and $575 \mathrm{~nm}$ with very low extinction coefficients [50]. The predicted peak is observed at $226 \mathrm{~nm}$ but there are very weak transitions at lower energy which are a better match for the experimental data. These can be seen at $409 \mathrm{~nm}$ and $517 \mathrm{~nm}$ if one zooms in on the visible region (Spectrum 11). When adjusted for the $0.22 \mathrm{eV}$ error these two peaks are shifted to $441 \mathrm{~nm}$ and $585 \mathrm{~nm}$. This is a reasonable agreement with experimental data. Remodelling in aqueous solution caused a negligible change to the position of the transitions. 


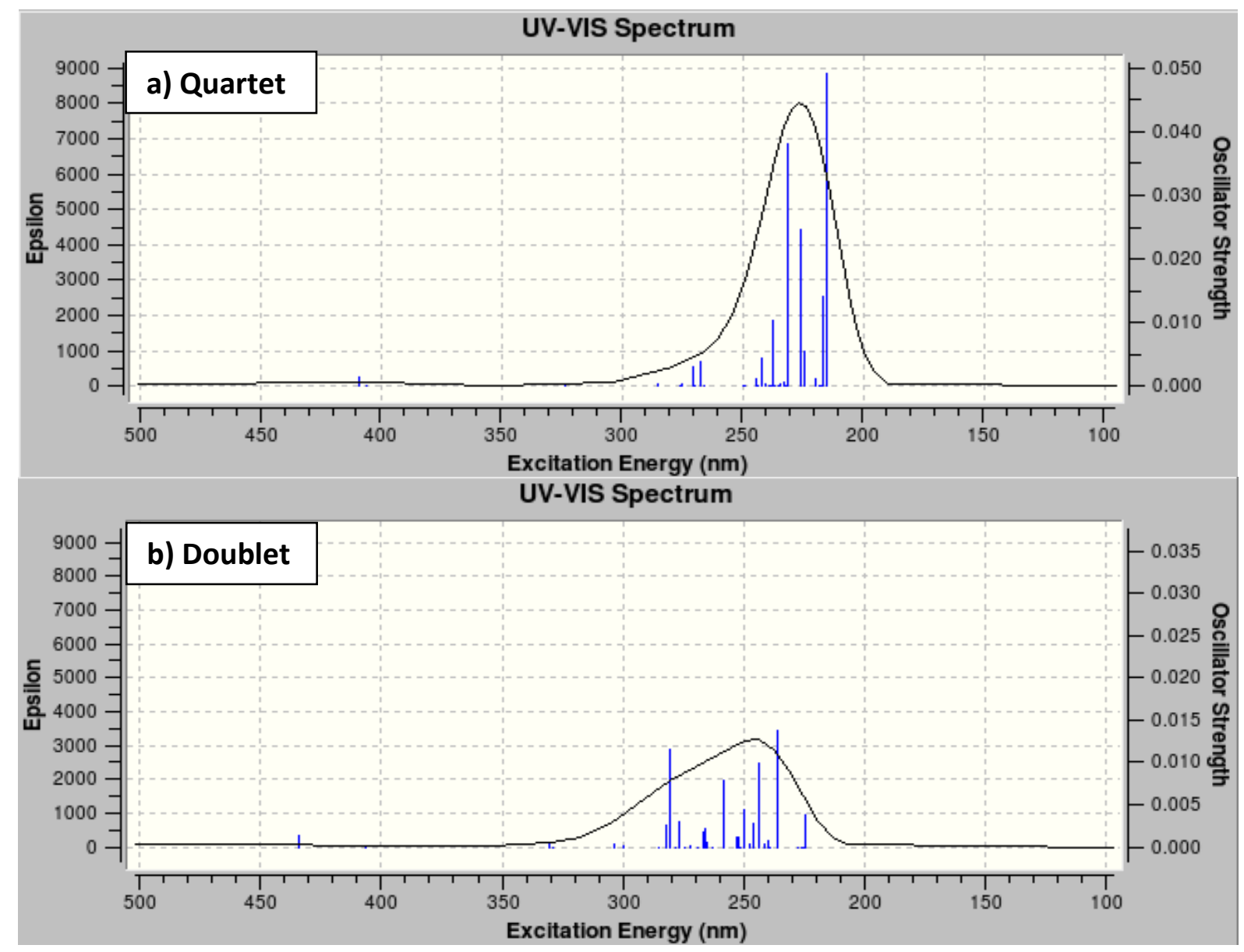

Spectrum 10: UV/Vis spectrum of trioxalatochromate

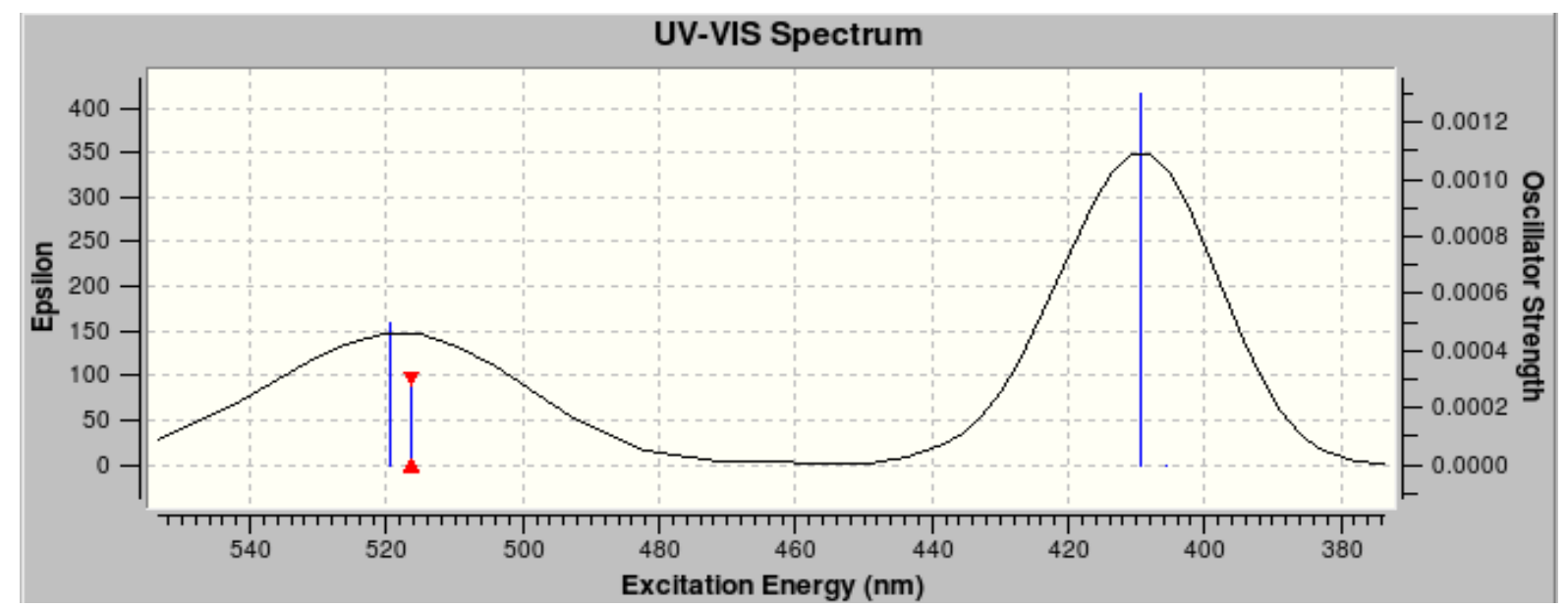

Spectrum 11: Zoom in on visible region of Spectrum 10a

The quartet first excited state configuration arises from 12 electronic configurations, but to simplify only the 8 configurations which contribute the most to the excited state are analysed. These contribute $84 \%$ of the excited state. There are two from HOMO-2(E) $\rightarrow$ LUMO+1(E) (12\%), two from HOMO-2(E) $\rightarrow$ LUMO+2(E) (14\%), two from $\mathrm{HOMO}(\mathrm{E}) \rightarrow \mathrm{LUMO}+1(\mathrm{E}) \quad(28 \%)$ and two from HOMO(E) $\rightarrow$ LUMO $+2(E) \quad(30 \%)$. 
Visual determination of the nature of the orbitals shows that one of the MOs in the HOMO-2 is bonding between the metal and all ligands. It is also bonding between C-C and between the non-coordinating oxygens within each ligand. The MO is bonding with respect to the coordinating $\mathrm{C}=\mathrm{O}$ bond but antibonding with respect to the non-coordinating $\mathrm{C}=\mathrm{O}$ bond. There is more MO density on the 02/O5 ligand than the other two (Figure 30d). The other $\mathrm{MO}$ in the HOMO-2 is similar but in this case there is more $\mathrm{MO}$ density on the other two ligands and much less on the $02 / 05$ ligand. Also in this $\mathrm{MO}$ the bonding mode between the non-coordinating oxygens and between the carbons is not present in the 02/05 ligand (Figure $30 \mathrm{~d}$ ). One of the MOs in the HOMO is antibonding between the metal and $\mathrm{O} 2, \mathrm{O}$, $\mathrm{O} 6$ and 07 , but is bonding between the metal and 03 and 04 . The 02/05 ligand has little MO density on it, and only displays a weak antibonding mode between the coordinating and non-coordinating oxygens. The other ligands show bonding between the carbons, and between the coordinating and non-coordinating oxygens through the p-orbital on the carbon, which also means that all $\mathrm{C}=\mathrm{O}$ modes are bonding (Figure $30 \mathrm{c}$ ). The other $\mathrm{MO}$ in the $\mathrm{HOMO}$ is bonding between metal and all coordinated oxygens except for $\mathrm{O} 3$ and $\mathrm{O} 4$, which are antibonding. The 02/05 ligand shows bonding between the carbons, and between the coordinating and non-coordinating oxygens through the p-orbital on the carbon, which also means that all $\mathrm{C}=\mathrm{O}$ modes are bonding in this ligand, while the $03 / 07$ and the $04 / 06$ ligands have the same bonding pattern but much more weakly (Figure 30c). One of the MOs in the LUMO+1 is antibonding between the metal and $02,03,04$ and 05 , but due to the angle of the p-orbitals on $\mathrm{O} 6$ and $\mathrm{O} 7$ there appears to be a very weak bonding interaction mitigating the antibonding interaction. There is almost no $\mathrm{MO}$ density on the $\mathrm{O} 2 / \mathrm{O} 5$ ligand, but plenty on the other two ligands. These two ligands show a strong C-C bonding interaction, and a twisted bonding interaction between the coordinating oxygens and the carbons due to the angle of the oxygen p-orbitals. The non-coordinating oxygens are antibonding to the carbons (Figure 30b). The other $\mathrm{MO}$ in the LUMO+1 is antibonding between the metal all ligands, but again there is a weak bonding interaction with respect to the $02 / 05$ ligand. The $02 / 05$ ligand shows a strong $\mathrm{C}-\mathrm{C}$ bonding interaction, and a twisted bonding interaction between the coordinating oxygens and the carbons due to the angle of 
a) $\mathrm{LUMO}+2$
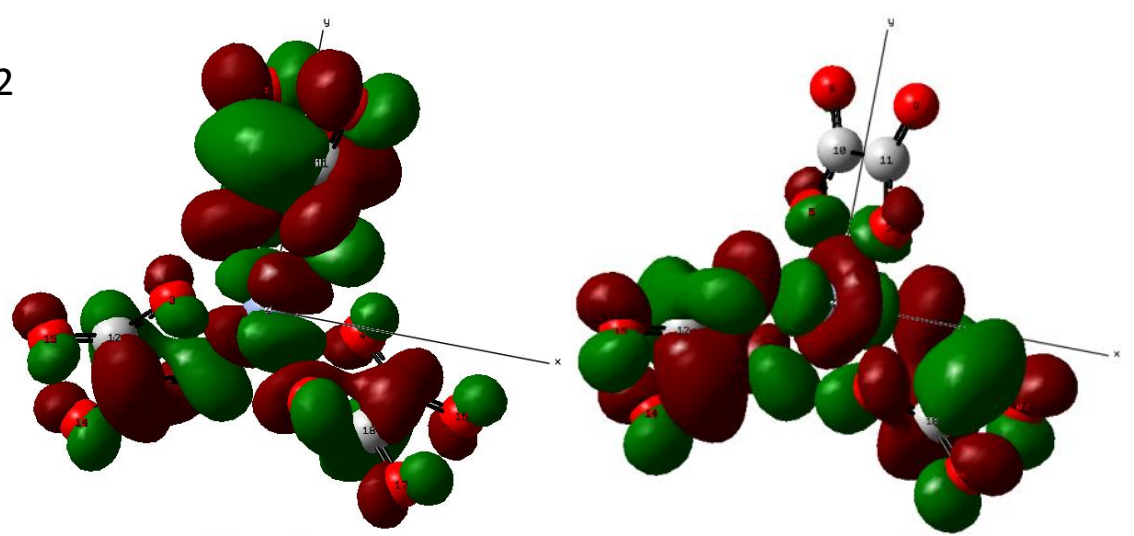

b) LUMO+1
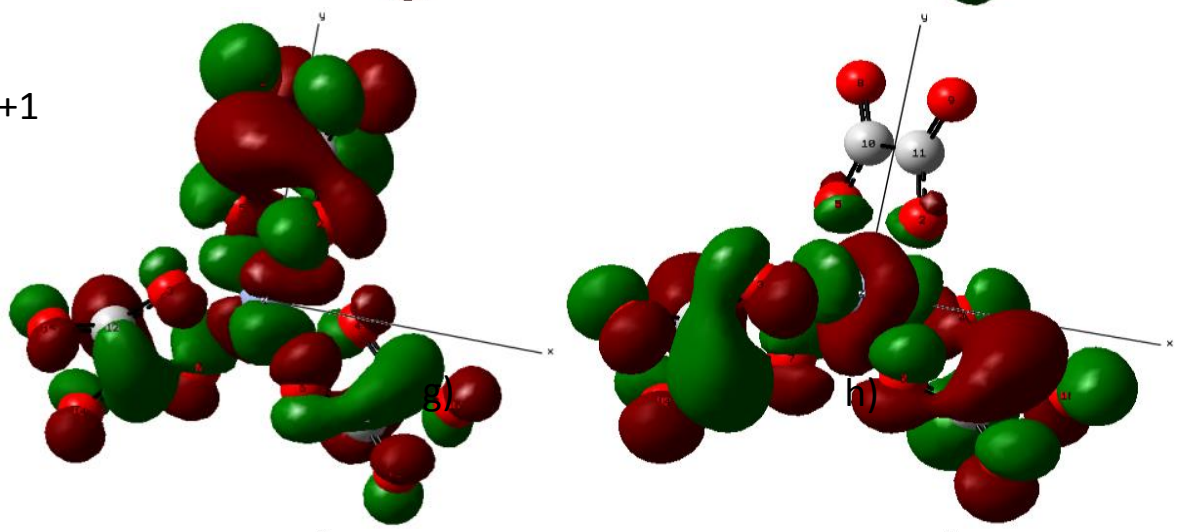

c) HOMO
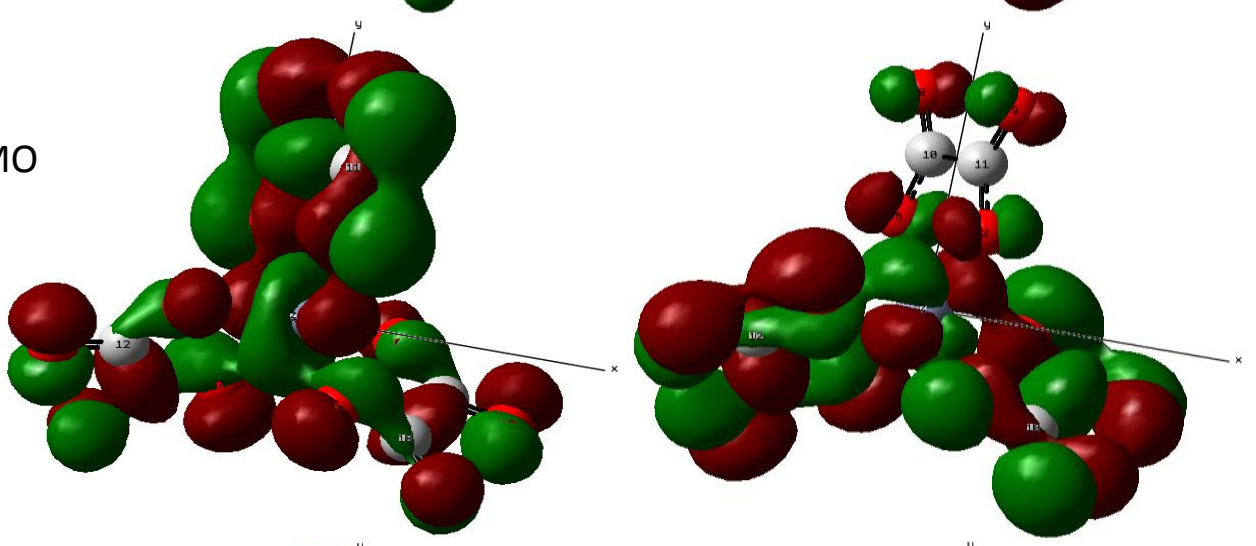

d) HOMO-2

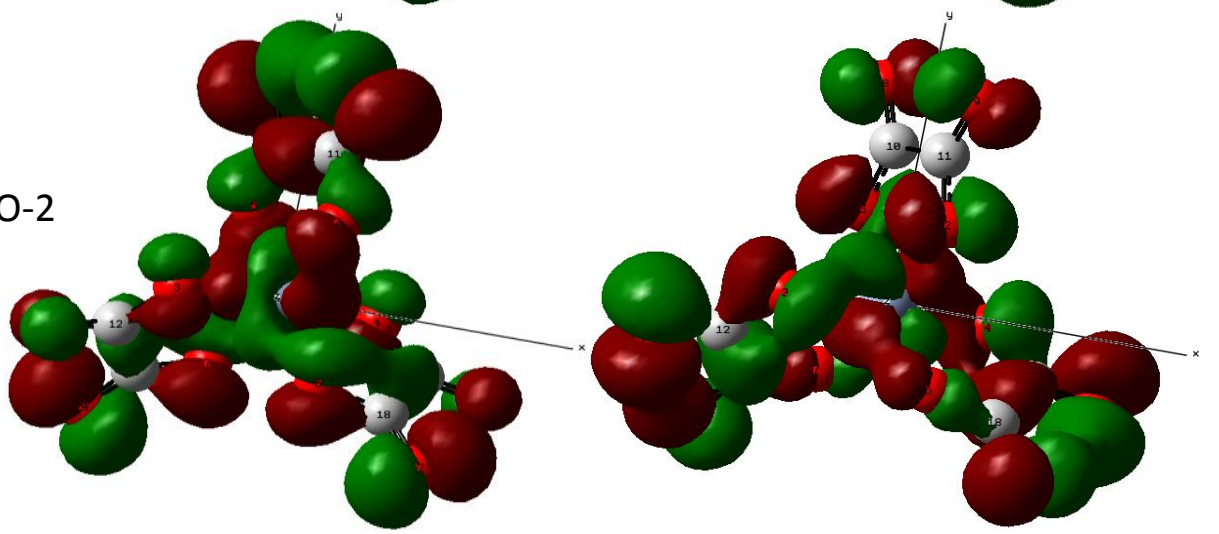

Figure 30: Relevant ground state MOs for $\left[\mathrm{Cr}\left(\mathrm{C}_{2} \mathrm{O}_{4}\right)_{3}\right]^{3-}$ quartet: a) LUMO+2 b) LUMO+1 c) HOMO d) HOMO-2 
the oxygen p-orbitals. The non-coordinating oxygens are antibonding to the carbons, and the other two ligands look the same but have much less density so will have weaker interactions (Figure 30b). LUMO+2 has an identical bonding pattern to LUMO+1 with the phases of the lobes reversed (Figure 30a).

In general, the excited state configuration has an antibonding interaction between the metal and ligands as this is the prevalent interaction observed in the LUMO+1 and LUMO+2. This will result in a physical change as the ground state configuration had mostly bonding metal-ligand interactions, and it is expected the ligands to shift further away from the metal centre in the ES. There will also be minor shifts in the intraligand bonding as the strong conjugated bonding modes observed in the HOMO-2 and HOMO are replaced with less conjugated bonding modes or antibonding interactions. Thus it is likely that the noncoordinating carbonyl bond will lengthen.

The optimised geometry of the quartet first excited state was calculated and found to have no symmetry. The $\mathrm{Cr}-\mathrm{O}$ bond distance varies from $1.91 \AA$ to $2.52 \AA$, the coordinated $\mathrm{O}-\mathrm{C}$ varies from 1.26 to $1.30 \AA$, the $\mathrm{C}$ - $\mathrm{C}$ varies from $1.57 \AA$ to $1.59 \AA$ and the non-coordinated $\mathrm{O}-\mathrm{C}$ varies from $1.23 \AA$ to $1.25 \AA$. The Mulliken charges are $\mathrm{Cr}=1.54$, coordinating $\mathrm{O}$ vary from 0.52 to -0.59 , C vary from 0.22 to 0.29 , and the other $O$ vary from -0.45 to -0.51 . This is close to predicted, as many of the ligands have moved further away from the metal, and we also see the non-coordinating carbonyl bond lengthen.

The vertical excitation energy for quartet trioxalatochromate was found to be $230 \mathrm{kJmol}^{-1}$. Vibrational relaxation decreased the energy by $45 \mathrm{kJmol}^{-1}$ so the energy difference between the quartet GS and ES was $185 \mathrm{kJmol}^{-1}$.

The vertical excitation energy for doublet trioxalatochromate was found to be $32 \mathrm{kJmol}^{-1}$. The optimised excited state for doublet trioxalatochromate could not be calculated as it repeatedly converged to an oscillation which was of too high an energy difference to consider a minimum. 


\section{Tris(ethylenediamine)chromate $\left[\operatorname{Cr}(e n)_{3}\right]^{3+}$}

The quartet ground state of tris(en)chromate was found to have $C_{2}$ symmetry (Figure 31 ). The $\mathrm{Cr}-\mathrm{N}$ bond distance was $2.14 \AA$. The $\mathrm{N}-\mathrm{C}$ bond distance was $1.51 \AA$ and the $\mathrm{C}-\mathrm{C}$ bond distance was $1.51 \AA$. The Mulliken charges are $\mathrm{Cr}=1.64$, $\mathrm{N}=-0.94$, and $\mathrm{C}=-0.30$.

The doublet lowest lying state was also found to have $C_{2}$ symmetry. This $C_{2}$ axis bisects the $\mathrm{C} 26-\mathrm{C} 29$ bond. The $\mathrm{Cr}-\mathrm{N}$ bond distance was $2.12 \AA$ for N9/N13, $2.13 \AA$ for N2/N11 and $2.14 \AA$ for N5/N7. The N-C bond distance is $1.51 \AA$ and the $\mathrm{C}-\mathrm{C}$ bond distance is $1.51 \AA$. The Mulliken charges are

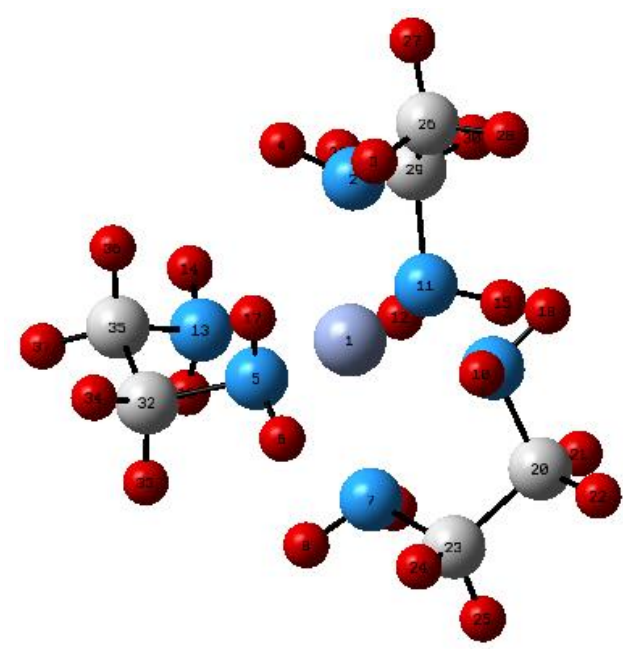

Figure 31: Tris(en)chromate $\mathrm{Cr}=1.62, \mathrm{~N}=-0.94, \mathrm{C}=-0.30$.

The predicted UV-Vis spectrum for tris(en)chromate has two main peaks at $221 \mathrm{~nm}$ with $\varepsilon$ of 9900 and at $166 \mathrm{~nm}$ with $\varepsilon$ of 18000 (Spectrum 12). The first excited state appears at 436 $\mathrm{nm}$ but is forbidden and so have a very low $\varepsilon$.

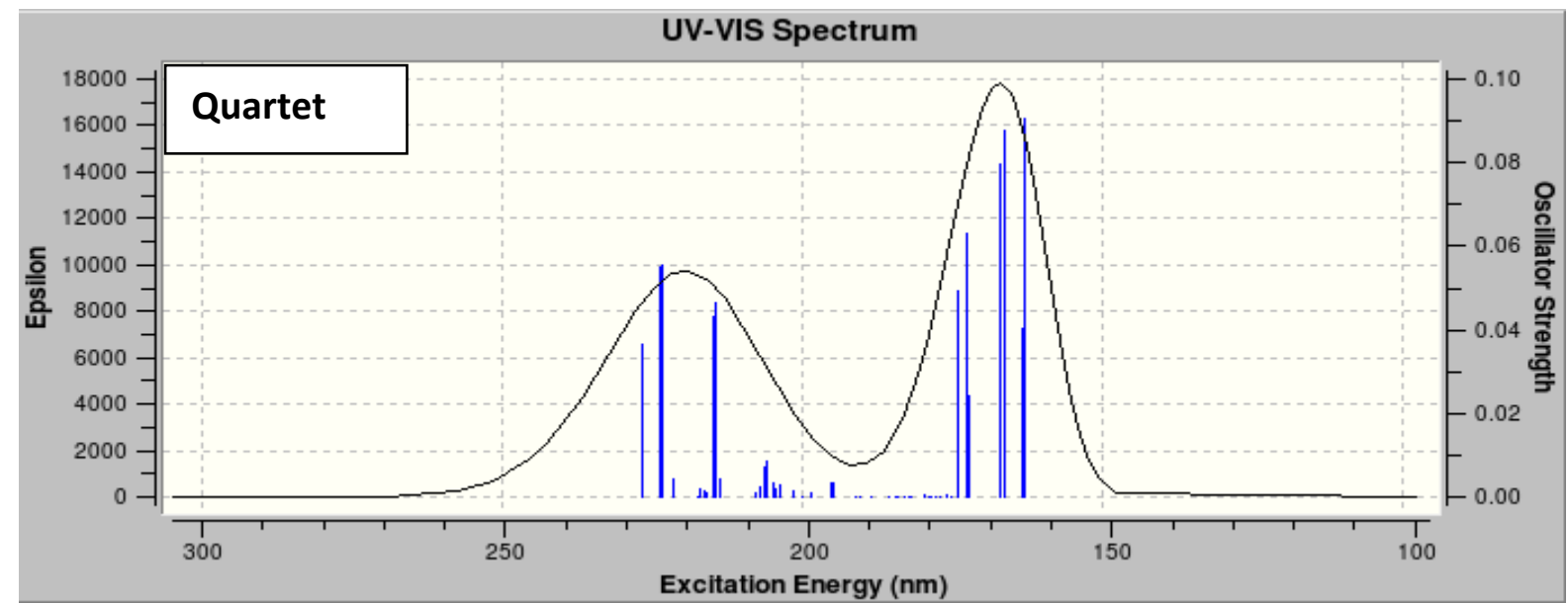

Spectrum 12: UV-Vis spectrum of tris(en)chromate

The experimental data for tris(en)chromate shows peaks at $350 \mathrm{~nm}$ and $457 \mathrm{~nm}$ with very small extinction coefficients [50]. The predicted peaks are at $166 \mathrm{~nm}$ and $221 \mathrm{~nm}$ but there are lower energy theoretical peaks with very low epsilon values which are a better match to experiment. These peaks appear at $356 \mathrm{~nm}$ and $435 \mathrm{~nm}$ and can be seen if one zooms in on the relevant area (Spectrum 13). When adjusted for the $0.22 \mathrm{eV}$ error these two peaks are 
shifted to $380 \mathrm{~nm}$ and $471 \mathrm{~nm}$ which is reasonable agreement with experiment. Remodelling in aqueous solution only causes a negligible change in the position of the transitions.

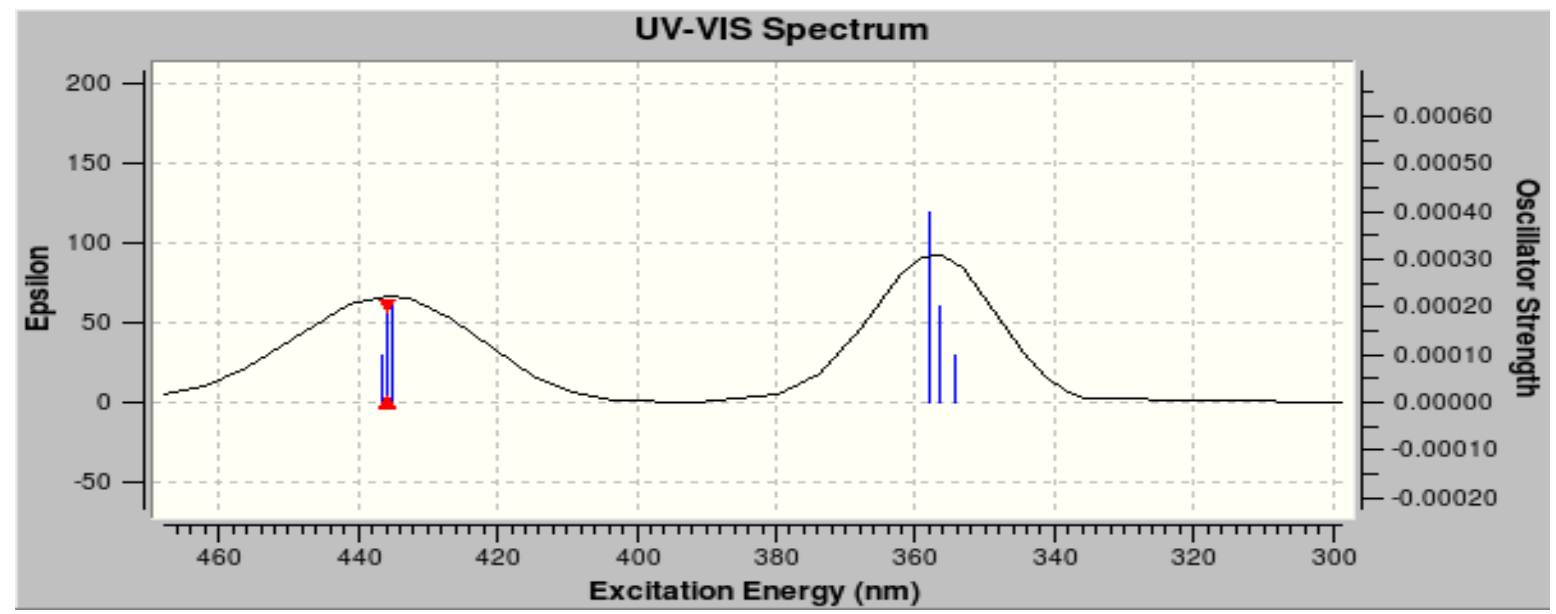

Spectrum 13: Zoom in on the UV-Vis spectrum of tris(en)chromate

The quartet first excited state configuration arises from 7 electronic configurations, of which there are three main configurations which together contribute $88 \%$ of the excited state.

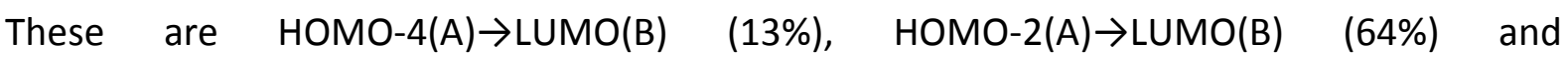
HOMO(A) $\rightarrow$ LUMO(B) (11\%). Visual determination of the nature of the molecular orbitals shows that the HOMO-4 is bonding between the metal and all ligands, through nitrogens N2, N5, N7 and N11, and non-bonding to N9 and N13. The N2/N11 ligand shows a bonding mode running the length of the $\mathrm{H}-\mathrm{N}-\mathrm{C}-\mathrm{C}-\mathrm{N}-\mathrm{H}$ chain, and is antibonding between the carbons and one of the methylene hydrogens. The other two ligands both show $\mathrm{H}-\mathrm{N}-\mathrm{C}-\mathrm{C}$ bonding on the side of the ligands which is coordinated, while the other $N$ in the ligand shows no bonding nature. These ligands also show the $\mathrm{C}-\mathrm{H}$ antibonding mode (Figure $32 \mathrm{~d}$ ). $\mathrm{HOMO}-2$ is weakly bonding between the metal and N2 and N11, but antibonding to the other nitrogens. The N2/N11 ligand shows $\mathrm{C}-\mathrm{C}$ bonding and $\mathrm{C}-\mathrm{N}$ bonding, and $\mathrm{N}-\mathrm{H}$ bonding while the other two ligands show $\mathrm{C}-\mathrm{N}$ bonding and $\mathrm{C}-\mathrm{C}$ antibonding as well as the same $\mathrm{C}-\mathrm{H}$ and $\mathrm{N}-\mathrm{H}$ bonding (Figure 32c). HOMO is bonding between the metal and all ligands through nitrogens $\mathrm{N} 2, \mathrm{~N} 5, \mathrm{~N} 7$ and $\mathrm{N} 11$ and weakly antibonding to $\mathrm{N} 9$ and N13. The N2/N11 ligand has N-H bonding, $\mathrm{C}-\mathrm{H}$ bonding and $\mathrm{C}-\mathrm{C}$ bonding but has $\mathrm{C}-\mathrm{N}$ antibonding. The other two ligands are $\mathrm{C}-\mathrm{N}$ bonding on the coordinated nitrogen and antibonding on the non-coordinated nitrogen, $\mathrm{C}-\mathrm{C}$ bonding and also shows some $\mathrm{C}-\mathrm{H}$ and $\mathrm{N}-\mathrm{H}$ bonding (Figure $32 \mathrm{~b}$ ). LUMO is antibonding 
between the metal and N2, N5, N7 and N11, and non-bonding to N9 and N13. It is also weakly bonding between N2, N5, N7 and N11 and the respective carbon atoms (Figure 32a).
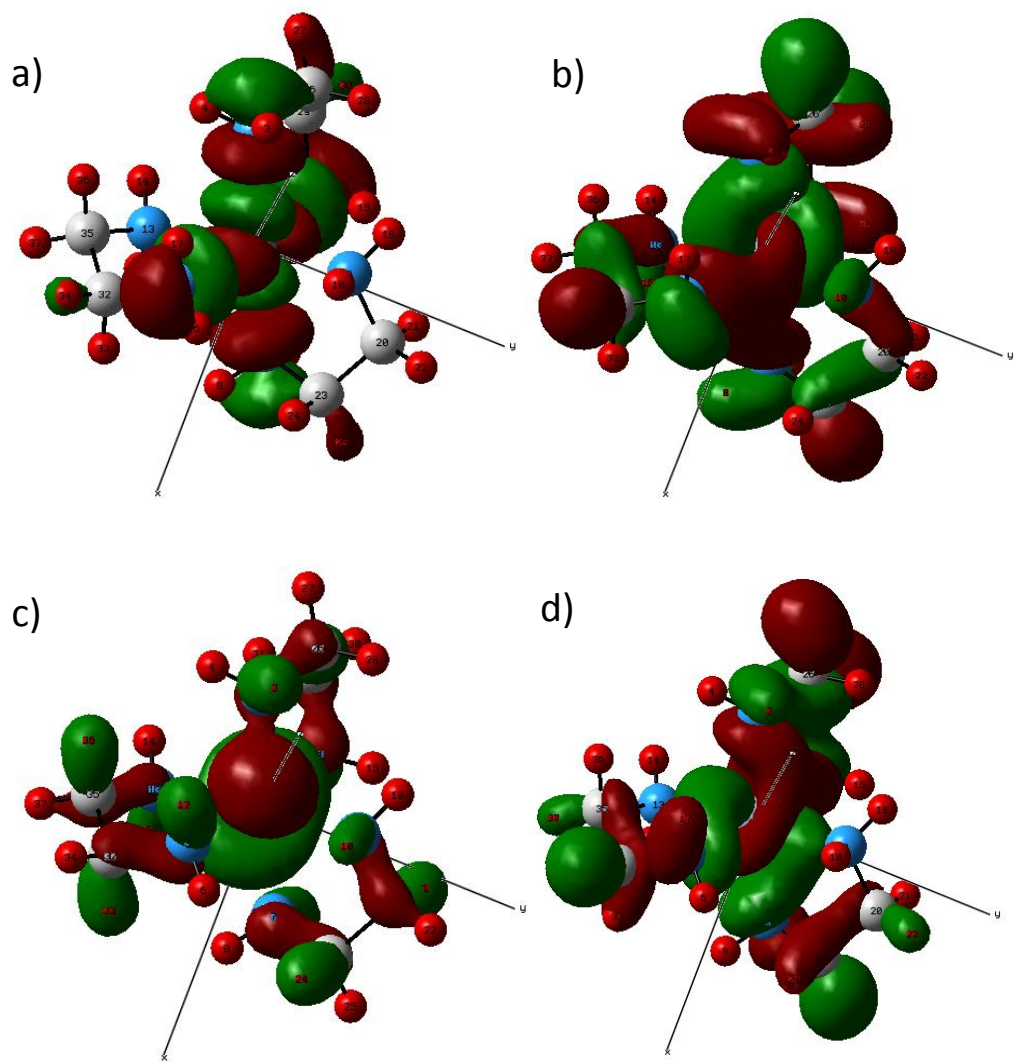

Figure 32: Relevant ground state MOs for tris(en)chromate quartet: a) HOMO-4 b) HOMO-2 c) HOMO d) LUMO

The HOMO-4 $\rightarrow$ LUMO configuration contributes only $13 \%$ to the excited state, and so will have a small effect on the excited state structure. These effects include the lengthening of the $\mathrm{Cr}-\mathrm{N}$ distance for $\mathrm{N} 2, \mathrm{~N} 5, \mathrm{~N} 7$ and N11 as these were bonding interactions in the HOMO4 but are antibonding in the LUMO. There will also be minor changes within the ligands, such as an elongation of the C-C and C-N bonds as the LUMO has almost no intraligand bonding. The HOMO $\rightarrow$ LUMO configuration only contributes $11 \%$ to the excited state and so will also have only a minor effect on the excited state. These effects are similar as for HOMO-4, and include the lengthening of the $\mathrm{Cr}-\mathrm{N}$ distance for N2, N5, N7 and N11 as these were bonding interactions in the HOMO but are antibonding in the LUMO, as well as intraligand effects such as a decrease in the N2-C and N11-C bond length due to the loss of the antibonding mode, and the subsequent lengthening of the $\mathrm{C}-\mathrm{C}$ bond in that ligand. The other two ligands will both show a decrease in the coordinated N-C bond length and an increase in the other $\mathrm{N}-\mathrm{C}$ bond length, as well as an elongation of the $\mathrm{C}-\mathrm{C}$ bond. The main 
configuration of these three is the HOMO-2 $\rightarrow$ LUMO, which contributes $64 \%$ to the excited state, and so will have a much larger effect on the structure of the excited state than the other two transitions. These effects should include an increase in the $\mathrm{Cr}-\mathrm{N} 2$ and $\mathrm{Cr}-\mathrm{N} 11$ distance as the bonding mode observed in HOMO-2 is replaced by an antibonding mode. Also, N9 and N13 should shift closer to the metal as the antibonding interaction is replaced by a non-bonding one. The intraligand changes will be similar to the other transitions. The N2/N11 ligand will show an increase in the C-C bond length and in general the hydrogens on ligands will all be less tightly bound. All structural changes appear to be $C_{2}$ symmetrical and so the excited state is expected to retain this symmetry.

The optimised geometry of the quartet first excited state was calculated and found to have retained $C_{2}$ symmetry as predicted. However, the molecule has undergone significant geometric changes as a result of the electronic transitions. The $\mathrm{Cr}-\mathrm{N}$ bond distance was 2.13 $\AA$ for $\mathrm{N} 9 / \mathrm{N} 13,2.40 \AA$ for $\mathrm{N} 2 / \mathrm{N} 11$ and $2.19 \AA$ for N5/N7. The N-C bond distance was $1.50 \AA$ for N2/N11, $1.52 \AA$ for N9/N13 and $1.51 \AA$ for N5/N7. The C-C bond distance is $1.51 \AA$. The Mulliken charges are $\mathrm{Cr}=1.65 ; \mathrm{N} 2 / \mathrm{N} 11=-0.91, \quad \mathrm{~N} 9 / \mathrm{N} 13=-0.94$ and $\mathrm{N} 5 / \mathrm{N} 7=-0.94$; $\mathrm{C} 26 / \mathrm{C} 29=-0.29, \quad \mathrm{C} 20 / \mathrm{C} 35=-0.29$ and $\mathrm{C} 23 / \mathrm{C} 32=-0.31$. This data supports the predictions made previously. Most importantly, we can clearly see a distortion of the $\mathrm{N}-\mathrm{Cr}-\mathrm{N}$ angle as the position of the ligands around the metal centre changes and exaggerates the $C_{2}$ symmetry (Figure 33).
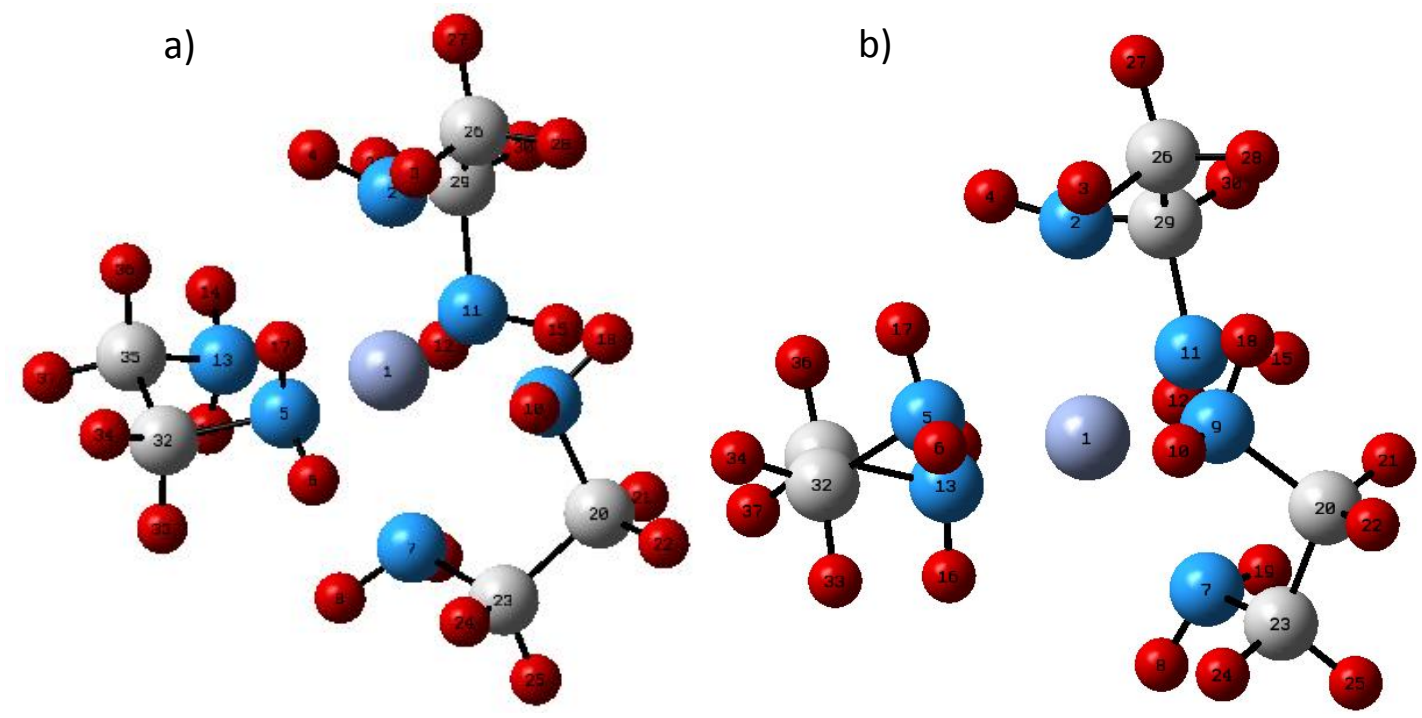

Figure 33: Optimised geometries of quartet tris(en)chromate: a) GS b) ES 


\section{Summary}

Some general trends can be observed in the collected data. For all the chromium compounds studied, as well as crystal violet, the energy gap for the first excitation from the ground state was much larger than the energy gap for the first excitation from the lowest lying state of different multiplicity. For crystal violet this means that the singlet state, with an $E_{v e r t}$ of $251 \mathrm{kJmol}^{-1}$ in the gas phase has a much larger energy gap than the triplet state, and in fact the triplet ground state is of a similar energy to the singlet first ES as shown in Figure 34. The triplet $E_{\text {vert }}$ is only $20 \mathrm{kJmol}^{-1}$ which is extremely small, and there is virtually no vibrational energy decrease meaning that the vertical excitation is already in the lowest vibrational state.

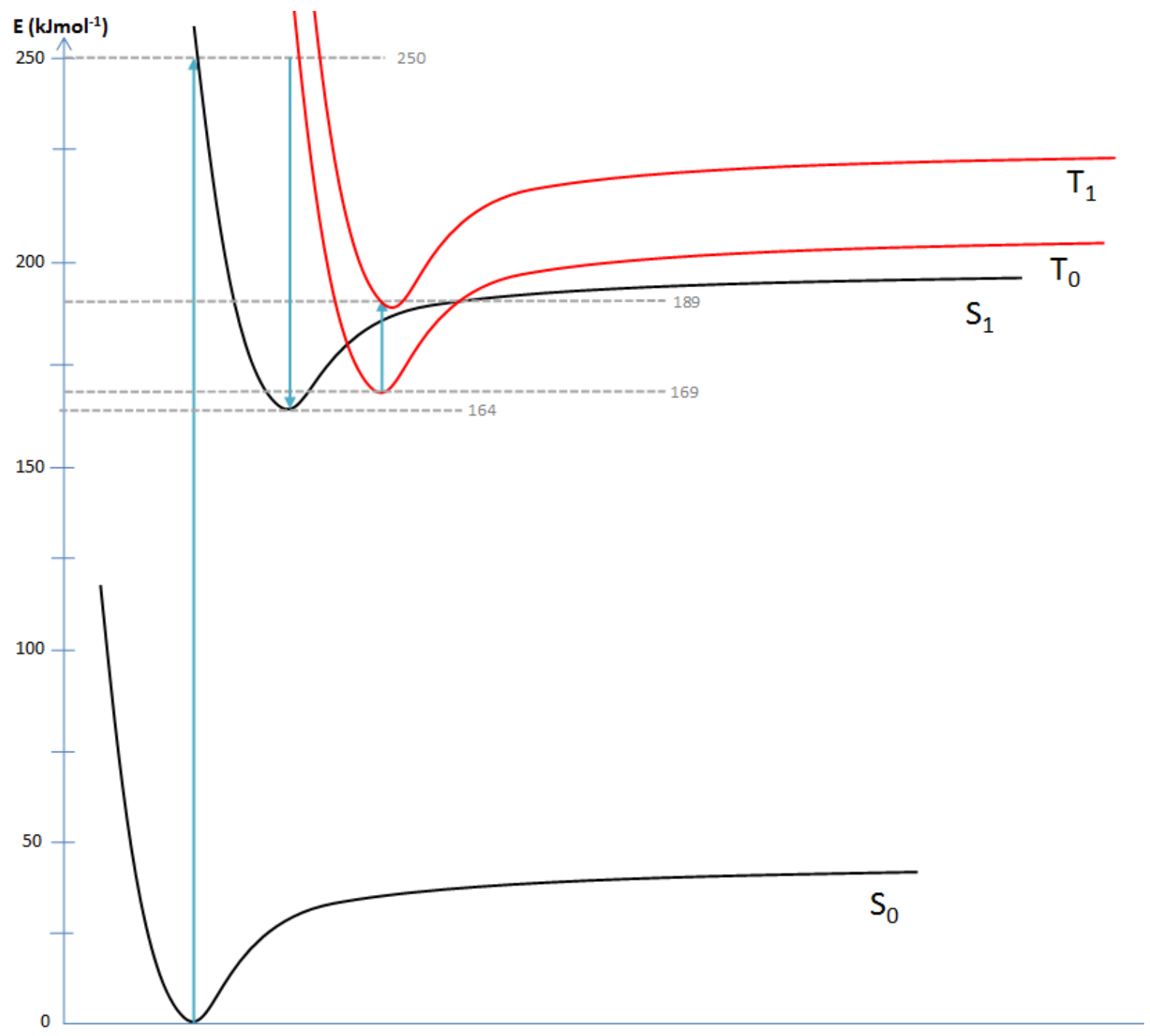

Figure 34: Relative energies of the singlet ground state and first excited state and the triplet lowest lying and first excited state of crystal violet. 
This small energy gap does not necessarily correspond to a small HOMO-LUMO gap however, as the electronic transition which gives rise to the triplet excited state configuration does not encompass the HOMO-LUMO gap. It is primarily the transition of a beta electron between the doubly occupied HOMO-2 and the singly occupied HOMO-1. This explains why the predicted triplet UV/Vis spectrum shows the first excited state at such low energy (almost 6000nm).

Also, if the $0.22 \mathrm{eV}$ error is consistent for the X3LYP method, then the corrected $E_{\text {vert }}$ is $230 \mathrm{kJmol}^{-1}$ for the singlet state in the gas phase. The singlet spectrum in aqueous solution shows the main peak shifted to $498 \mathrm{~nm}$ which corresponds to an energy of $240 \mathrm{kJmol}^{-1}$. This corrects to an $E_{\text {vert }}$ of $219 \mathrm{kJmol}^{-1}$, which corresponds to a singlet absorbance peak at $547 \mathrm{~nm}$. This is a reasonable match to the experimental data peak of $590 \mathrm{~nm}$ in aqueous solution.

In the singlet crystal violet we see a prime example how differences in electronic configuration can affect a physical change, as the transitions from HOMO $\rightarrow$ LUMO cause a major shift of electron density onto the central carbon decreasing the positive charge by 0.29 and actually making the central carbon slightly negative. There is also a significant rotation of one of the arms of the molecule relative to the molecular plane. This causes a major destabilisation in the molecule due to the loss of conjugation through the central carbon which is why there is such a large energy difference between the GS and ES.

The triplet crystal violet also undergoes a geometric change upon excitation, whereby the arms of the molecule rotate so that the angle between the phenyl rings and the molecular plane is more similar to the singlet GS. Arms $A$ and $C$ decrease the angle by about $5-9^{\circ}$ and Arm B increases its angle by roughly the same amount.

The chromium compounds also exhibit a few trends, as all the ligand systems studied showed that the doublet $E_{v e r t}$ is considerably smaller than the quartet $E_{v e r t}$ and that in fact the energy gap for the quartet states encompasses the two doublet states (Figure 35). This small gap between the doublet states matches nicely with the known small energy difference as seen in the Tanabe-Sugano diagram for $d^{3}$ octahedral complexes (Figure 2) [35]. 

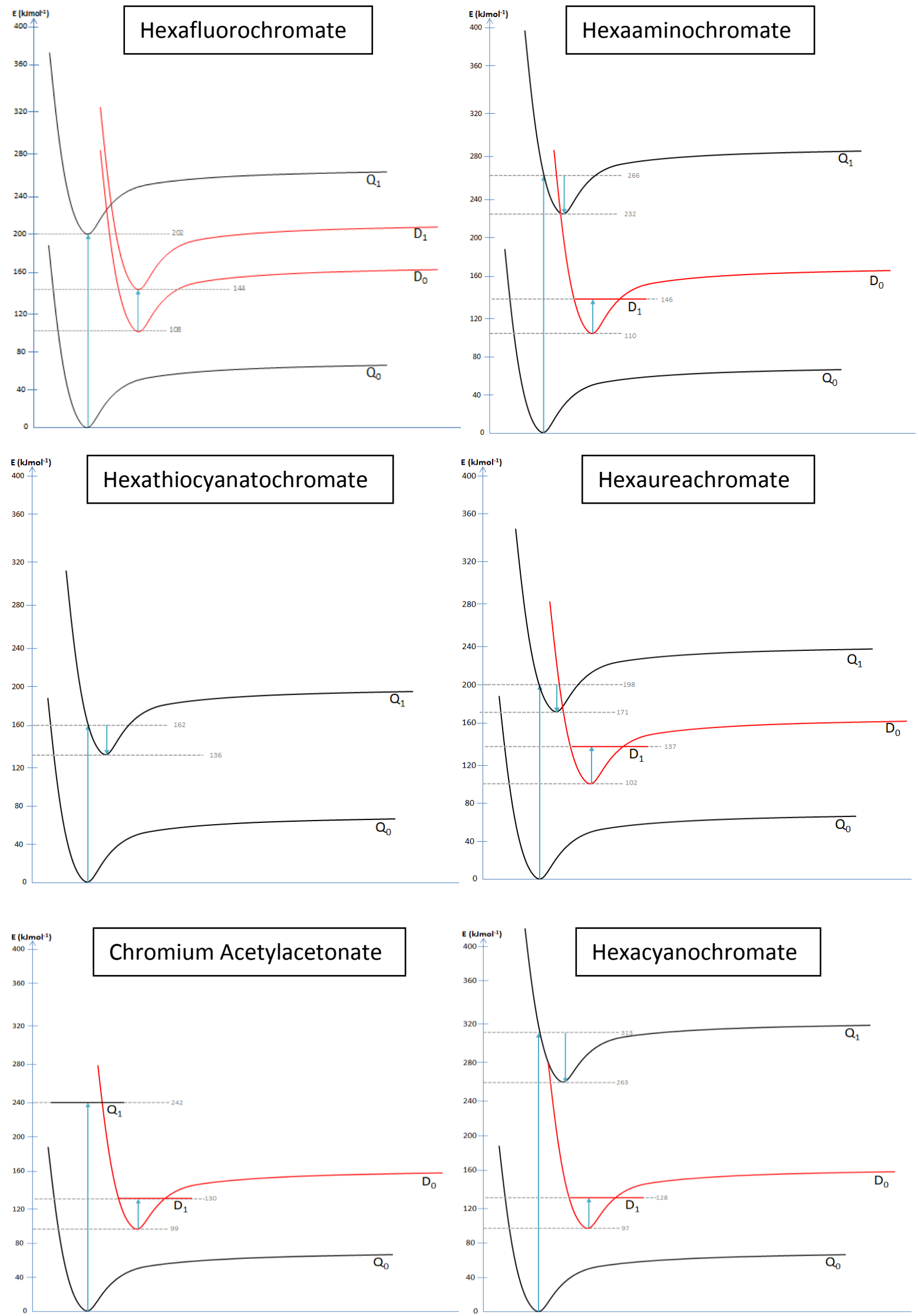

63 

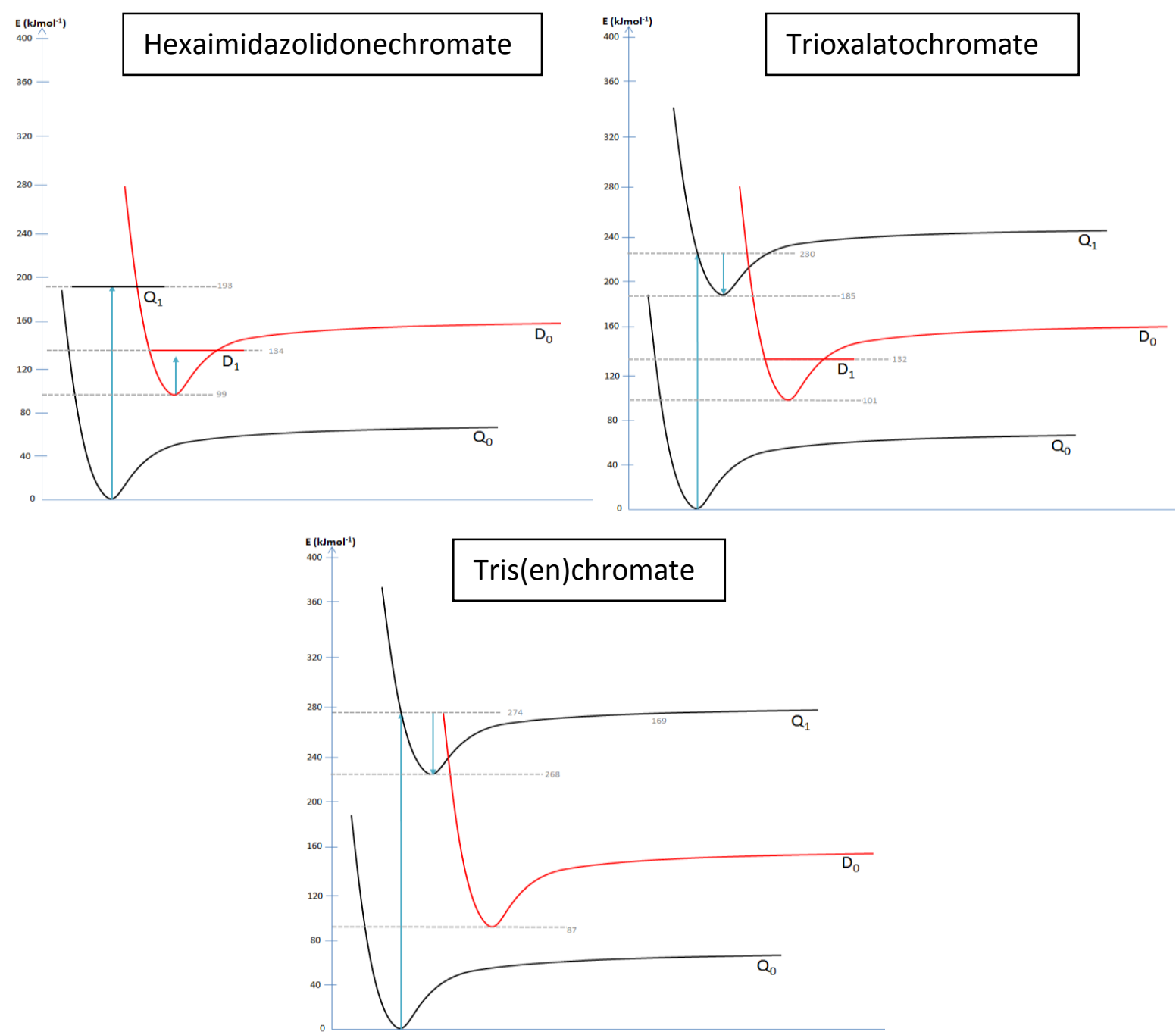

Figure 35: Relative energies of the quartet ground state and first excited state and the doublet lowest lying and first excited state of a range of chromium compounds in the gas phase. (NB: a flat line shows $\mathrm{E}_{\text {vert }}$ and indicates that the optimised geometry of the ES could not be calculated)

The comparison between predicted spectra and experimental spectra proved to be reasonably accurate. The molecules' predicted spectra were all within a reasonable margin of error of the experimental data after adjustment for the knows $+0.22 \mathrm{eV}$ error in the method, with chromium acetylacetonate providing the closest match to experiment, and hexacyanochromate the worst match. 


\section{Conclusion}

Overall, this work has shown the effectiveness of TD-DFT for modelling electronic excitations within a known range of error, and as an extremely useful tool for predicting structural and geometric effects as a result of electronic excitation using molecular orbitals. It also shows the limits of the X3LYP method compared to more accurate, but more computationally expensive methods.

The crystal violet molecule is shown to undergo a definite geometrical change upon excitation to the first excited state. For the singlet this occurs through the loss of a 'twisting' bonding mode which allows one of the arms of the molecule to relax out of the known $D_{3}$ symmetry. The triplet lowest lying state also undergoes a geometric change upon excitation in a similar fashion, resulting in a rotation of the arms of the molecule, however the triplet excitation is extremely low in energy as it is an excitation of minority spin between occupied orbitals.

The chromium compounds analysed show the difficulty in calculating excited states for complicated electronic systems such as metal complex ions. This is evident in the dearth of optimised excited state structures which were obtained. However, in general the spectroscopic prediction was shown to be reasonable for most of the molecules, and the utilisation of the molecular orbitals for prediction of the structural features of the excited states was shown to be effective for the molecules of which the excited state structure could be calculated. The main difficulty in this method of prediction is the multireference character which is prevalent in most of the chromium compounds, as their excited state configurations are almost all the result of contributions from multiple electronic configurations. Further analysis in this area was prevented due to time constraints but it would have provided a fuller picture to obtain models for both bonding types for hexathiocyanatochromate, and also to look more closely at the lower energy $d$ - $d$ transitions which generally seem to have very low $\varepsilon$ in these compounds. It is this area of the spectra that the experimental data is mainly focussed on.

This general methodology could be applied to a range of molecular systems, though clearly it is more effective in modelling simpler systems such as organic dyes, and it would be an interesting course of future study to analyse not just the first excited states, but the states 
which are highly absorbing in the lower wavelength region. As these excitations are allowed and strongly absorbing, understanding the structures which result from these excitations may provide interesting applications. 


\section{References}

1. $\quad$ Atkins P, d.P.J., Physical Chemistry. 9th ed2010: Oxford University Press.

2. D, P., Introduction to spectroscopy. 4th ed2009, Belmont, CA: Brooks/Cole, Cengage learning.

3. Hohenberg, P. and W. Kohn, Inhomogeneous electron gas. Physical review, 1964. 136(3B): p. B864.

4. Chai, S., et al., Multiple hydrogen bonding in excited states of aminopyrazine in methanol solution: Time-dependent density functional theory study. Spectrochim. Acta, Part A, 2013. 115: p. 39-44.

5. Hedrick, M.M., et al., First-Principles Studies of the Ground-and Excited-State Properties of Quantum Dots Functionalized by Ru(II)-Polybipyridine. J. Phys. Chem. C, 2013. 117: p. 1821618224.

6. Wei, M.-j., D.-q. Jia, and F.-w. Chen, Geometric structures, excitation energies and dipole moments of the ground and excited states of TiO2. Wuli Huaxue Xuebao, 2013. 29: p. 14411452.

7. Frank, J., Introduction to computational chemistry. 2nd ed2007: Wiley.

8. Löwdin, P.-O., Quantum Theory of Many-Particle Systems. III. Extension of the Hartree-Fock Scheme to Include Degenerate Systems and Correlation Effects. Physical review, 1955. 97(6): p. 1509-1520.

9. Runge, E. and E.K. Gross, Density-functional theory for time-dependent systems. Physical Review Letters, 1984. 52(12): p. 997.

10. Casida, M.E., Time-dependent density functional response theory for molecules. Vol. 1. 1995: World Scientific: Singapore.

11. Bonvoisin, J. and I. Ciofini, Spectral signature of a Ru(II, III, IV) complex: a combined experimental and theoretical investigation. Dalton Trans., 2013. 42: p. 7943-7951.

12. Camino, B., L.P.M. De, and A.M. Ferrari, Photoelectrochemical properties of the CT1 dye: A DFT study. J. Mol. Struct., 2013. 1046: p. 116-123.

13. Das, S., et al., A Combined Experimental and DFT/TD-DFT Investigation of Structural, Electronic, and Cation-Induced Switching of Photophysical Properties of Bimetallic Ru(II) and Os(II) Complexes Derived from Imidazole-4,5-Dicarboxylic Acid and 2,2'-Bipyridine. Inorg. Chem., 2013. 52: p. 6860-6879.

14. Fukuda, R. and M. Ehara, Electronic excited states and electronic spectra of biphenyl: a study using many-body wavefunction methods and density functional theories. Phys. Chem. Chem. Phys., 2013. 15: p. 17426-17434.

15. Li, W.-b. and Y.-m. Yuan, Theoretical calculation of molecular spectroscopy of 5-methyl-3nitro-4-isoxazole formyl hydrazone. Fenzi Kexue Xuebao, 2013. 29: p. 237-245.

16. Tessaro, A.L., et al., Electronic structures and spectroscopic properties of benzoporphyrin protolytic species: A TD-DFT study. Comput. Theor. Chem., 2013. 1020: p. 173-179.

17. Yang, D.-P., Y.-G. Yang, and Y.-F. Liu, Theoretical study on hydrogen bonding of mono- and dihydrated complexes of 7-(3'-pyridyl)indole in excited states. J. Chin. Chem. Soc. (Weinheim, Ger.), 2013. 60: p. 618-624.

18. Lee, S.U., Influence of exchange-correlation functional in the calculations of vertical excitation energies of halogenated copper phthalocyanines using time-dependent density functional theory (TD-DFT). Bull. Korean Chem. Soc., 2013. 34: p. 2276-2280.

19. Dhouib, A., et al., DFT and Ab Initio calculations of spectroscopic properties of tetramethyltin and of its cation. Int. J. Quantum Chem., 2012. 112: p. 2032-2042.

20. Garino, C. and L. Salassa, The photochemistry of transition metal complexes using density functional theory. Philos. Trans. R. Soc., A, 2013. 371: p. 20120134/1-20120134/24.

21. Boo, B.H. and J.H. Kim, Fluorescence and fluorescence excitation spectroscopy of 5,8dihydroxy-1,4-naphthoquinone. Analysis of the electronic spectra via the time-dependent DFT calculation. Bull. Korean Chem. Soc., 2013. 34: p. 309-312. 
22. Shen, L., Theoretical investigation on the triplet excited state properties of the porphyrinrelated photosensitizers and the implications in illustrating their photosensitization mechanisms. J. Mol. Struct.: THEOCHEM, 2008. 862: p. 130-132.

23. Yang, D.-P., H. Li, and Y.-F. Liu, A TD-DFT study on the effect of intermolecular hydrogen bonding on the photophysical properties of N-methyl-1,8-naphthalimide. J. Chin. Chem. Soc. (Weinheim, Ger.), 2013. 60: p. 267-274.

24. Goddard, W.A. First-principles multiscale multiparadigm methods for applications to energy production, storage, and utilization. 2013. American Chemical Society.

25. Olguin, M., R.R. Zope, and T. Baruah, The effect of structural conformational changes on charge transfer states in a light-harvesting carotenoid diaryl-Porphyrin-C60 molecular triad. arXiv.org, e-Print Arch., Condens. Matter, 2013: p. 1-17, arXiv:1308.2000v1 [cond-mat.mtrlsci].

26. Shuai, Z.G., et al., Theoretical study of the low-lying electronic excited states for molecular aggregates. Sci. China: Chem., 2013. 56: p. 1258-1262.

27. Zhao, J., et al., Triplet photosensitizers: from molecular design to applications. Chem. Soc. Rev., 2013. 42: p. 5323-5351.

28. Cañamares, M.V., et al., DFT, SERS, and Single-Molecule SERS of Crystal Violet. The Journal of Physical Chemistry C, 2008. 112(51): p. 20295-20300.

29. Meng, W., et al., SERS and DFT study of crystal violet. Journal of Molecular Structure, 2013. 1035(0): p. 326-331.

30. Liang, E.J., X.L. Ye, and W. Kiefer, Surface-Enhanced Raman Spectroscopy of Crystal Violet in the Presence of Halide and Halate lons with Near-Infrared Wavelength Excitation. The Journal of Physical Chemistry A, 1997. 101(40): p. 7330-7335.

31. Sugar, J. and C.H. Corliss, Energy levels of chromium, Cr I through Cr XXIV1977: American Chemical Society and the American Institute of Physics for the National Bureau of Standards.

32. DeArmond, K. and L.S. Forster, Electronic transitions in chromium (III) complexes-l: Absorption spectra of 6-diketone chelates. Spectrochimica Acta, 1963. 19(9): p. 1393-1401.

33. Dingle, R., Crystal Spectra of Hexaurea Complexes. II. Absorption and Emission in Crystals Containing the Hexaurea Chromium (III) Ion. The Journal of Chemical Physics, 1969. 50: p. 1952.

34. Tanaka, N., et al., Infrared Spectroscopic Studies of Hexammine-and Pentammine-chromium (III) Complexes. Bulletin of the Chemical Society of Japan, 1964. 37(2): p. 222-225.

35. Tanabe, Y. and S. Sugano, On the absorption spectra of complex ions. J. Phys. Soc. Jpn, 1954. 9(5): p. 753-766.

36. Gaussian 09, Revision B.01, M. J. Frisch, G. W. Trucks, H. B. Schlegel, G. E. Scuseria,M. A. Robb, J. R. Cheeseman, G. Scalmani, V. Barone, B. Mennucci, G. A. Petersson, H. Nakatsuji, M. Caricato, X. Li, H. P. Hratchian, A. F. Izmaylov, J. Bloino, G. Zheng, J. L. Sonnenberg, M. Hada, M. Ehara, K. Toyota, R. Fukuda, J. Hasegawa, M. Ishida, T. Nakajima, Y. Honda, O. Kitao, H. Nakai, T. Vreven, J. A. Montgomery, Jr., J. E. Peralta, F. Ogliaro, M. Bearpark, J. J. Heyd, E. Brothers, K. N. Kudin, V. N. Staroverov, T. Keith, R. Kobayashi, J. Normand, K. Raghavachari, A. Rendell, J. C. Burant, S. S. lyengar, J. Tomasi, M. Cossi, N. Rega, J. M. Millam, M. Klene, J. E. Knox, J. B. Cross, V. Bakken, C. Adamo, J. Jaramillo, R. Gomperts, R. E. Stratmann, O. Yazyev, A. J. Austin, R. Cammi, C. Pomelli, J. W. Ochterski, R. L. Martin, K. Morokuma, V. G. Zakrzewski, G. A. Voth, P. Salvador, J. J. Dannenberg, S. Dapprich, A. D. Daniels, O. Farkas, J. B. Foresman, J. V. Ortiz, J. Cioslowski, and D. J. Fox, Gaussian, Inc., Wallingford CT, 2010..

37. $\mathrm{Xu}, \mathrm{X}$. and W.A. Goddard, The X3LYP extended density functional for accurate descriptions of nonbond interactions, spin states, and thermochemical properties. Proceedings of the National Academy of Sciences of the United States of America, 2004. 101(9): p. 2673-2677.

38. Laurent, A.D. and D. Jacquemin, TD-DFT benchmarks: A review. International Journal of Quantum Chemistry, 2013. 
39. Dennington, R.K., T; MIllam, J, Gaussview, 2009, Semichem Inc: Shawnee Mission KS.

40. Tomasi, J., B. Mennucci, and E. Cancès, The IEF version of the PCM solvation method: an overview of a new method addressed to study molecular solutes at the QM ab initio level. Journal of Molecular Structure: THEOCHEM, 1999. 464(1-3): p. 211-226.

41. Kleinman, S.L., et al., Single-Molecule Surface-Enhanced Raman Spectroscopy of Crystal Violet Isotopologues: Theory and Experiment. J. Am. Chem. Soc., 2011. 133(11): p. 41154122.

42. Skoog D.A, W.D.M., Holler J, Analytical Chemistry: An Introduction. 7th Ed ed. Saunders Golden Sunburst Series1999.

43. Bhasikuttan, A.C., L.V. Shastri, and A.V. Sapre, On the formation of triplet state of crystal violet in solutions - a pulse radiolysis study. Radiation Physics and Chemistry, 1997. 49(1): p. 35-37.

44. Jacquemin, D., et al., Extensive TD-DFT benchmark: singlet-excited states of organic molecules. J. Chem. Theory Comput., 2009. 5(9): p. 2420-2435.

45. Pierloot, K. and L. Vanquickenborne, The ligand field spectrum of the hexafluorochromate (III) anion: An ab initio study including correlation effects. The Journal of Chemical Physics, 1990. 93: p. 4154.

46. Schlaefer, H.L., H. Gausmann, and H.U. Zander, Luminescence behavior and light absorption of some fluoro complexes of tervalent chromium. Inorganic Chemistry, 1967. 6(8): p. 15281532.

47. Alexander J.L. Hoppé M.A. Malati, V., Solvent effects on the electronic spectrum of the hexathiocyanatochromate (III) anions. A revised octahedral crystal field splitting parameter for the thiocyanate ion. Polyhedron, 1982. 1(2): p. 191-193.

48. Hanuza, J., et al., Spectroscopic properties of hexacyanochromate(3-) ion trapped in sodium chloride, potassium chloride, rubidium chloride, and potassium bromide hosts. J. Mol. Struct., 1989. 212(Copyright (C) 2013 American Chemical Society (ACS). All Rights Reserved.): p. 25569.

49. Chatterjee, K.K. and G.B. Porter, Spectra of hexaimidazolidonechromium(III) and tris(biuret)chromium(III) perchlorates. Inorg. Chem., 1966. 5: p. 860-3.

50. Mead, A., Absorption spectra, in aqueous solution, of co-ordination compounds of chromium and cobalt. Transactions of the Faraday Society, 1934. 30: p. 1052-1058. 